DESY 06-209

Archive: quant-ph/0612032

\title{
A New Look at the
}

\section{Quantum Mechanics of the Harmonic Oscillator}

\author{
H.A. Kastrup 1 \\ DESY, Theory Group \\ Notkestr. 85, D-22603 Hamburg \\ Germany
}

PACS 03.65.Fd, 03.65.Ge, 42.50.Xa

\begin{abstract}
In classical mechanics the harmonic oscillator (HO) provides the generic example for the use of angle and action variables $\varphi \in \mathbb{R} \bmod 2 \pi$ and $I>0$ which played a prominent role in the "old" Bohr-Sommerfeld quantum theory. However, already classically there is a problem which has essential implications for the quantum mechanics of the $(\varphi, I)$-model for the HO: the transformation $q=\sqrt{2 I} \cos \varphi, p=-\sqrt{2 I} \sin \varphi$ is only locally symplectic and singular for $(q, p)=(0,0)$. Globally the phase space $\{(q, p)\}$ has the topological structure of the plane $\mathbb{R}^{2}$, whereas the phase space $\{(\varphi, I)\}$ corresponds globally to the punctured plane $\mathbb{R}^{2}-(0,0)$ or to a simple cone with the tip deleted. From the properties of the symplectic transformations on that phase space one can derive the functions $h_{0}=I, h_{1}=I \cos \varphi$ and $h_{2}=-I \sin \varphi$ as the basic coordinates on $\{(\varphi, I)\}$, where their Poisson brackets obey the Lie algebra of the symplectic group of the plane. This implies a qualitative difference as to the quantum theory of the phase space $\{(\varphi, I)\}$ compared to the usual one for $\{(q, p)\}$ : In the quantum mechanics for the $(\varphi, I)$-model of the HO the three $h_{j}$ correspond to the self-adjoint generators $K_{j}, j=0,1,2$, of certain irreducible unitary representations of the symplectic group or one of its infinitely many covering groups, the representations being parametrized by a (Bargmann) index $k>0$. This index $k$ determines the ground state energy $E_{k, n=0}=\hbar \omega k$ of the $(\varphi, I)$-Hamiltonian $H(\vec{K})=\hbar \omega K_{0}$. For an $m$-fold covering the lowest possible value for $k$ is $k=1 / m$, which can be made arbitrarily small by choosing $m$ accordingly! This is not in contradiction to the usual approach in terms of the operators $Q$ and $P$ which are now expressed as functions of the $K_{j}$, but keep their usual properties. The richer structure of the $K_{j}$ quantum model of the HO is "erased" when passing to the simpler $(Q, P)$-model! This more refined approach to the quantum theory of the HO implies many experimental tests: Mulliken-type experiments for isotopic diatomic molecules, experiments with harmonic traps for atoms, ions and BE-condensates, with charged HOs in external electric fields and the (Landau) levels of charged particles in external magnetic fields, with the propagation of light in vacuum, passing through strong external electric or magnetic fields. Finally it may lead to a new theoretical estimate for the quantum vacuum energy of fields and its relation to the cosmological constant.
\end{abstract}

\footnotetext{
${ }^{1}$ E-mail: Hans.Kastrup@desy.de
} 


\section{Contents}

1 Introduction and overview

1.1 The issue: Quantum mechanics of the harmonic oscillator in terms of angle

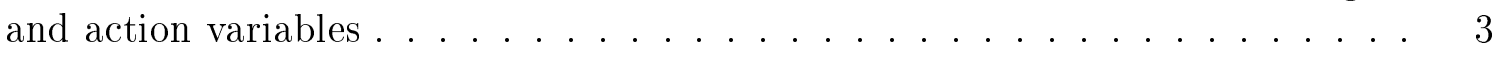

1.2 Contents overview . . . . . . . . . . . . . . . . . . . . . 8

1.3 Possible experiments . . . . . . . . . . . . . . . . . 10

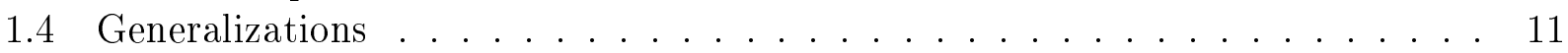

1.5 Range of the paper .......................... 12

2 Some properties of the classical harmonic oscillator $\quad \mathbf{1 2}$

2.1 The globally singular relationship between the canonical pairs $(q, p)$ and $(\varphi, I) \quad 12$

2.2 A symplectic scale transformation . . . . . . . . . . . . . . . 14

2.3 Going beyond the harmonic oscillator . . . . . . . . . . . . . . 15

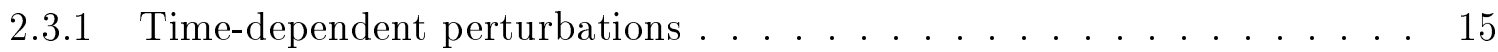

2.3.2 Interactions proportional to $\tilde{h}_{1}$ or $\tilde{h}_{2} \ldots \ldots \ldots \ldots$

2.3.3 Morse and other "integrable" potentials ............. 16

2.3.4 Free non-relativistic particle ................ 18

3 Action of the symplectic group on the phase space $\mathcal{S}_{\tilde{q}, \tilde{p}} \quad \mathbf{1 9}$

3.1 Global and infinitesimal transformations, "observables" . . . . . . . . . . . 19

3.2 Vector fields and their associated Hamiltonian functions . . . . . . . . . . . . 20

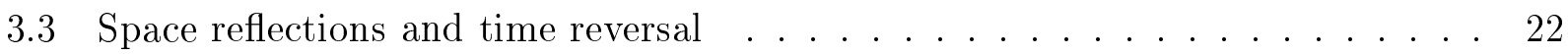

3.4 The space $\mathcal{S}_{\tilde{q}, \tilde{p} ; 0}$ as a "homogeneous" one $\ldots \ldots \ldots \ldots \ldots$

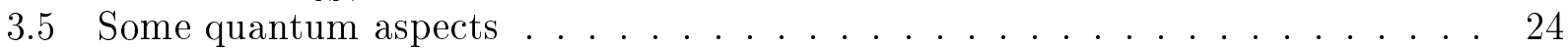

4 Action of the proper orthochronous homogeneous Lorentz group in $1+2$ dimensions on the phase space $\mathcal{S}_{\varphi, \tilde{I}}$

4.1 The basic canonical "observables" on $\mathcal{S}_{\varphi, \tilde{I}} \ldots \ldots \ldots \ldots \ldots$

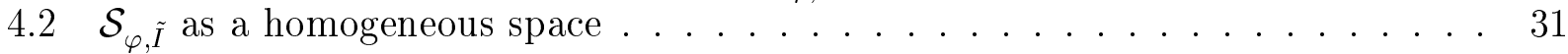

4.3 On the relationship between the phase spaces $\mathcal{S}_{\tilde{q}, \tilde{p} ; 0}$ and $\mathcal{S}_{\varphi, \tilde{I}} \ldots \ldots \ldots$

4.4 Relationships between the coordinates $\tilde{q}, \tilde{p}$ and $\tilde{h}_{0}, \tilde{h}_{1}, \tilde{h}_{2} \ldots \ldots . \ldots 3$

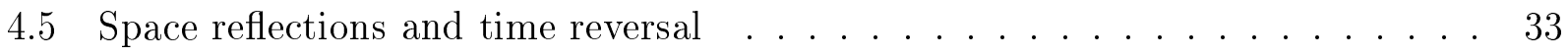

5 Quantizing the angle - action variables phase space $\mathcal{S}_{\varphi, \tilde{\mathbf{I}}}$ of the harmonic oscillator

5.1 Lie algebra of the self-adjoint observables $\tilde{K}_{j}$ and the structure of their irre-

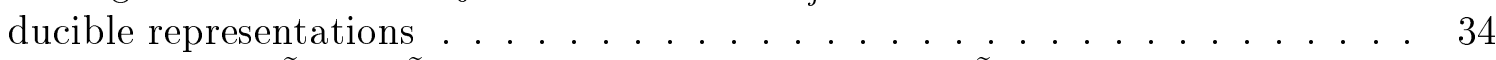

5.2 The operators $\tilde{Q}$ and $\tilde{P}$ as functions of the operators $\tilde{K}_{j} \ldots \ldots . \ldots$

5.2.1 Operator version of the polar coordinates in the plane . . . . . . . . 37

5.2.2 Two kinds of energy spectra for the quantum mechanical HO . . . . . 38

5.2.3 Time evolution and the ground states for different covering groups . . . 39

5.2.4 The index $k$ in number states matrix elements . . . . . . . . . . . 40

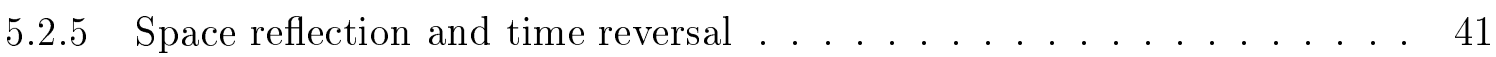

5.3 Restoring the physical dimensions . . . . . . . . . . . . . . . 42 
6 Three types of coherent states 42

6.1 Definition and physical interpretation . . . . . . . . . . . 42

6.1.1 Barut-Girardello coherent states . . . . . . . . . . . . . . 43

6.1.2 Perelomov coherent states . . . . . . . . . . . . . . . 44

6.1.3 Schrödinger-Glauber coherent states . . . . . . . . . . . . 44

6.1.4 Physical interpretation of the complex variables . . . . . . . . 44

6.2 Generation from the ground state . . . . . . . . . . . . . 46

6.2 .1 Schrödinger-Glauber states . . . . . . . . . . . . . 46

6.2 .2 Perelomov states . . . . . . . . . . . . . . . 46

6.2 .3 Barut-Girardello states . . . . . . . . . . . . . . . . . 47

6.2.4 Transitions between Perelomov and Barut-Girardello coherent states . . 48

6.3 Time evolution . . . . . . . . . . . . . . . . . . . . . . . 49

6.4 Some general properties $\ldots \ldots \ldots \ldots \ldots \ldots \ldots \ldots$

6.4 .1 Scalar products . . . . . . . . . . . . . . . . 49

6.4 .2 Completeness ... . . . . . . . . . . . . . . . 50

6.4.3 Hilbert spaces of holomorphic functions associated with the three types of coherent states . . . . . . . . . . . . . . 50

6.4.4 Probabilities for transitions to number states . . . . . . . . . . 53

6.5 Physical dynamics described by the basic operators $\tilde{K}_{0}, \tilde{K}_{+}$and $\tilde{K}_{-} \ldots \ldots .54$

6.5.1 Generation of Perelomov coherent states . . . . . . . . . . . 54

6.5.2 One-mode generated Lie algebra $\mathfrak{s o}(1,2) \ldots \ldots \ldots \ldots \ldots$

6.5.3 Interactions bilinear in the $K_{j} \ldots \ldots \ldots \ldots \ldots \ldots \ldots$

6.5.4 Two-mode generated Lie algebra $\mathfrak{s o}(1,2) \ldots \ldots \ldots \ldots \ldots$

6.5.5 Generation of Barut-Girardello coherent states . . . . . . . . . . . 57

6.5.6 Holstein-Primakoff type generators . . . . . . . . . . . . 57

6.5.7 Additional proposals for using symplectic groups in quantum optics . . 57

7 Examples of explicit Hilbert spaces for the $(\varphi, \mathbf{I})$-model of the harmonic oscillator $\quad 57$

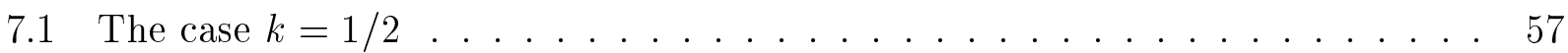

7.1.1 The Hardy space $H_{+}^{2}$ on the circle as the Hilbert space for the HO . . . 57

7.1 .2 Space reflections and time reversal . . . . . . . . . . . 60

7.1 .3 Perturbations . . . . . . . . . . . . . . . . . . 61 61

7.1 .4 A unitary transformation . . . . . . . . . . . . . . . 62

7.1.5 Coherent state wave functions and their probability densities . . . . . 62

7.1.6 Expectation values and transition probabilities . . . . . . . . 65

7.1 .7 Eigenfunctions of $\tilde{K}_{1}$ and $\tilde{K}_{2} \ldots \ldots \ldots \ldots 6 \ldots \ldots 6$

7.1.8 Relationship to the conventional description of the HO on $L^{2}(\mathbb{R}, d x)$. . 68

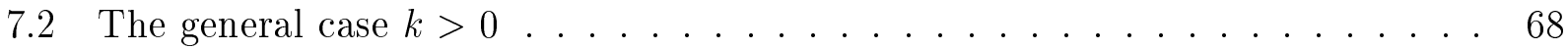

7.2.1 Hilbert space of holomorphic functions on the unit disc . . . . . . 69

7.2.2 Hilbert spaces related to the Hardy space on the circle . . . . . . . 70

7.2 .3 Hilbert space on the positive real line . . . . . . . . . . . 72

8 On the ground state of the quantized free electromagnetic field in a cavity $\mathbf{7 5}$

8.1 The electromagnetic field in a cavity as a set of harmonic oscillators . . . . 75

8.2 The cosmological constant problem . . . . . . . . . . . 77

8.3 Birefringence and dichroism of the vacuum $\ldots \ldots \ldots \ldots$ 
8.4 "Dark" normal matter? . . . . . . . . . . . . . . . . . . . 79

9 Charged particles in external electric and magnetic fields 79

9.1 Charged harmonic oscillator in an external electric field . . . . . . . . . . . 79

9.2 Charged particle in an external magnetic field . . . . . . . . . . . 80

10 Thermodynamics 82

Acknowledgements

$\begin{array}{ll}\text { Appendices } & 83\end{array}$

A Calculating the action variables for certain potentials of 1-dimensional systems

$\mathrm{B}$ The covering groups of $\mathrm{SO}^{\uparrow}(1.2)$ and the positive discrete series of their irreducible unitary representations 84

B.1 The universal covering group of $S O^{\uparrow}(1,2) \ldots \ldots \ldots \ldots \ldots \ldots$. . . . . . . .

B.2 Irreducible unitary representations of the positive discrete series for $k>0 \ldots 86$

C Estimates for the ratios $I_{2 k}(2|z|) / I_{2 k-1}(2|z|)$ of modified Bessel functions of the first kind for $k>0$

\section{Introduction and overview}

\subsection{The issue: Quantum mechanics of the harmonic oscillator in terms of angle and action variables}

At first sight it probably appears provocative and presumptuous to present a new research paper on the harmonic oscillator ( $\mathrm{HO}$ ), that venerable and pedagogically thoroughly squeezed simple model, encountered in many physics publications of all types. Despite its simplicity it has played an important role at many instances in the history of physics, classically and quantum theoretically:

It probably started with Hooke's law

$$
\dot{p}=-b q, p=M \dot{q}, b>0,
$$

in mechanics for the force exerted on a particle in the neighbourhood of its stable equilibrium position. Then came the HO in the plane with its two qualitatively different types of motion, periodical orbits (Lissajous figures) and quasi-periodical ones which densely fill a submanifold of the phase space, initiating the idea of ergodic systems. Two or more linearly coupled HO with their characteristic (eigen-) frequencies are important for the stability analysis of many systems and play a significant role in crucial areas of physics. By adding a friction term the model serves also as an examplary introduction to dissipative systems.

Conceptionally important was - and still is - the locally canonical (symplectic) description of the position and momentum coordinates for the HO in terms of angle and action variables:

$$
q(\varphi, I)=\sqrt{\frac{2 I}{M \omega}} \cos \varphi, \quad p(\varphi, I)=-\sqrt{2 M \omega I} \sin \varphi, \quad \omega=\sqrt{b / M},
$$


so that

$$
H(q, p)=\frac{1}{2 M} p^{2}+\frac{1}{2} M \omega^{2} q^{2}=H(\varphi, I)=\omega I .
$$

This is the generic example for the essential concept of integrable systems, their (nonintegrable) perturbations and the associated KAM-theory [1-3].

Then there is the possible interpretation of classical free electromagnetic standing waves in a cavity as a set of uncoupled harmonic oscillators. This property was essential in Planck's derivation of his radiation law. So the HO played an important part in the birth of quantum theory, too!

In the "old" quantum mechanics with its Bohr-Sommerfeld framework the HO had the energy levels $E_{n}=\hbar \omega n, n=0,1, \ldots$. (For a comprehensive summary of the Bohr-Sommerfeld theory, where the angle and especially the action variables played a central role, just before the dawn of modern quantum mechanics see the impressive textbook by Born (and Hund) [4].)

Even before Heisenberg deduced the modified energy levels

$$
E_{n}=\hbar \omega\left(n+\frac{1}{2}\right), \quad n=0,1, \ldots
$$

in his famous first paper on matrix mechanics [5], Mulliken had concluded from his spetroscopic analysis of the differences in the vibrational spectra of the diatomic isotopes $B^{10} O^{16}$ and $B^{11} O^{16}[6,7]$ that the lowest energy state of the HO should be

$$
E_{0}=\frac{1}{2} \hbar \omega
$$

This has been the canonical undisputed ground state energy value of the HO ever since (for a comprehensive historical overview see Ref. [8]) and a standard example for the role of Heisenberg's position - momentum uncertainty relations. For a recent partial survey of the HO in modern physics see Ref. [9].

Whereas angle and action variables were central "observables" in the old quantum mechanics, they disappeared almost entirely in the new quantum mechanics from 1925/26 on and the usage of the operators $Q$ and $P$ took over nearly completely. Dirac's early attempts [10] to use angle and action operators also for the new framework turned out to be contradictory, as pointed out by London [11] and Jordan [12] and the subject has remained controversial even up to now [13]. Before taking up that issue again, a few remarks as to the central role the ground state energy (5) started to play:

Around 1930 F. London deduced the van der Waals forces from the ground state energies of two 3-dimensional HOs [14].

The value (5) became a nuisance (and still is!), however, when free fields were quantized, because their interpretations as a set of an infinite number of HOs implied an (unobserved) infinite ground state energy. The problem has been "swept under the rug" by ignoring the ground state energies, formally by introducing "normal-ordering" for the associated annihilation and creation operators $a$ and $a^{\dagger}$ (see below).

Nevertheless the ground state energy (5) plays a very stimulating part in the discussions of the Casimir effect $[8,15,16]$ and also in the present attempts to understand the dark energy in the universe and the extremely obnoxious cosmological constant problem [17-24].

So the energy (5) is discarded or advocated depending on the physical concepts which are being discussed. Not a very convincing situation! 
In view of the general acceptance of the value (5) it is amazing that there appear to be no systematic modern experimental tests - similar to those of Mulliken - of such a conceptually important physical quantity! More on the experimental situation in subsec. 1.3 below.

It is one aim of the present paper to point out that the canonized ground state energy value (5) may not be the only possible one for the $\mathrm{HO}$, but that there is a canonical structure for the $\mathrm{HO}$ in terms of angle and action variables $\varphi$ and $I$ the quantum mechanics of which allows for ground state values

$$
E_{k, n=0}=\hbar \omega k, \quad k>0,
$$

where $k$ may be any positive number, especially an arbitrary small one $>0$ !

I ask for a moment of patience for the justification of this seemingly outrageous claim!

The main reason for the possibility (6) is the difference as to the global structures of the locally canonically (symplectically) equivalent phase spaces $\mathcal{S}_{q, p}$ and $\mathcal{S}_{\varphi, I}$ of the respective canonical pairs $(q, p)$ and $(\varphi, I)$ :

$$
\begin{gathered}
\mathcal{S}_{q, p}=\left\{(q, p) \in \mathbb{R}^{2}\right\}, \\
\mathcal{S}_{\varphi, I}=\{(\varphi, I), \varphi \in \mathbb{R} \bmod 2 \pi, I>0\},
\end{gathered}
$$

which shows that $\mathcal{S}_{q, p}$ has the global topological structure of the plane $\mathbb{R}^{2}$, whereas $\mathcal{S}_{\varphi, I}$ has that of a simple cone with the tip deleted or that of a punctured plane $\mathbb{R}^{2}-\{0\} \cong S^{1} \times \mathbb{R}^{+}$, where $S^{1}$ denotes the unit circle and $\mathbb{R}^{+}$the positive real numbers without the 0 .

This implies that $\mathcal{S}_{\varphi, I}$ cannot be quantized in the conventional manner in terms of the (Born-Heisenberg-Jordan-Dirac-) Weyl group generated by the 3-dimensional Lie algebra basis $\{q, p, 1\}$, but one has to pass to the 3-dimensional (proper orthochronous homogeneous Lorentz) group $S O^{\uparrow}(1,2)$ (in one "time" and two "space" dimensions) or to one of its (infinitely) many covering groups [13], among which the symplectic group $S p(2, \mathbb{R})$ in the $(q, p)$ plane is a double covering (like the group $S U(2)$ is a double covering of the rotation group $S O(3))$. That symplectic group provides the key to an appropriate quantization of the phase space (8) and plays an essential role in what follows.

The crucial point is that both the phase space $\mathcal{S}_{\varphi, I}$ and and its "canonical group" $S O^{\uparrow}(1,2)$ contain the topological "factor" $S^{1}$ which is multiply connected (with homotopy group $\pi_{1}\left(S^{1}\right)=$ $\mathbb{Z})$. This multi-connectedness has implications for the infinite-dimensional irreducible unitary representations of the non-compact group $S O^{\uparrow}(1,2)$ and its infinitely many covering groups because now the self-adjoint generator of the rotations $S O(2)$ can have more complicated spectra with a ground state like (6) . And this generator is proportional to the Hamilton operator of the HO in the $(\varphi, I)$-framework! (For the similar case of a simple rotator see Ref. [25].)

The transformation (2) from the space (8) onto the space (7) with its origin deleted is not special for the HO. It can be used for any $(1+1)$-dimnsional system with periodic motions in (17) describable by angle and action variables in (8). So their quantum mechanics is affected, too! Examples are discussed in subsec. 2.3.

Quantizing the phase space $\mathcal{S}_{\varphi, I}$ makes use of the positive discrete series $D_{k}^{(+)}, k>0$, of those unitary representations mentioned above $[13,26]$. In these representations the selfadjoint generator $K_{0}$ of the compact rotation subgroup $S O(2) \cong S^{1}$ constitutes the quantized counterpart of the classical action variable $I$ and the "boost" generators $K_{1}$ and $K_{2}$ correspond to the classical quantities $I \cos \varphi$ and $-I \sin \varphi$, the knowledge of which allows to determine the angle $\varphi \in(-\pi, \pi]$ uniquely. The choice of these basic "observables" on the phase space 
(8) can be justified systematically from the action of the symplectic group $S p(2, \mathbb{R})$ on the phase space (7). That action leaves the origin of the space (7) invariant!

Those basic classical observables

$$
h_{0}(\varphi, I)=I, \quad h_{1}(\varphi, I)=I \cos \varphi, \quad h_{2}(\varphi, I)=-I \sin \varphi,
$$

on $\mathcal{S}_{\varphi, I}$ obey the Lie algebra $\mathfrak{s o}(1,2)$ of the group $S O^{\uparrow}(1,2)$ and its (infinitely many) covering groups in terms of Poisson brackets:

$$
\left\{h_{0}, h_{1}\right\}_{\varphi, I}=-h_{2}, \quad\left\{h_{0}, h_{2}\right\}_{\varphi, I}=h_{1}, \quad\left\{h_{1}, h_{2}\right\}_{\varphi, I}=h_{0},
$$

where

$$
\left\{h^{(1)}, h^{(2)}\right\}_{\varphi, I} \equiv \partial_{\varphi} h^{(1)}(\varphi, I) \partial_{I} h^{(2)}(\varphi, I)-\partial_{I} h^{(1)}(\varphi, I) \partial_{\varphi} h^{(2)}(\varphi, I) .
$$

The corresponding quantum mechanical counterparts, the dimensionless self-adjoint operators

$$
\tilde{K}_{j}=K_{j} / \hbar
$$

obey

$$
\left[\tilde{K}_{0}, \tilde{K}_{1}\right]=i \tilde{K}_{2}, \quad\left[\tilde{K}_{0}, \tilde{K}_{2}\right]=-i \tilde{K}_{1}, \quad\left[\tilde{K}_{1}, \tilde{K}_{2}\right]=-i \tilde{K}_{0}
$$

For the positive discrete series the operator $\tilde{K}_{0}$ in general has the spectrum (eigenvalues)

$$
\sigma\left(\tilde{K}_{0}\right)=\left\{n+k, n=0,1, \ldots ; k \in \mathbb{R}^{+}\right\}
$$
$k$ are

For the $m$ th covering group $S O_{[m]}^{\uparrow}(1,2), m=1,2, \ldots$, of $S O^{\uparrow}(1,2)$ the allowed values of

$$
k=\frac{\mu}{m}, \mu \in \mathbb{N}=\{1,2, \ldots\},
$$

so that the smallest attainable value of $k$ for a corresponding irreducible unitary representation is

$$
k=\frac{1}{m}
$$

As $m$ can be an arbitrarily large natural number, $k$ can be made arbitrarily small $>0$ !

The quantum mechanical $(q, p)$-Hamiltonian

$$
H(q, p) \rightarrow H(Q, P)=\frac{1}{2 M} P^{2}+\frac{1}{2} M \omega^{2} Q^{2}=-\frac{\hbar^{2}}{2 M} \frac{d^{2}}{d q^{2}}+\frac{1}{2} M \omega^{2} q^{2}
$$

has the unambiguous spectrum (44). However, in view of Eq. (14) the quantum mechanical $(\varphi, I)$-Hamiltonian

$$
H(\varphi, I) \rightarrow H(\vec{K})=\omega K_{0}, \quad \vec{K}=\hbar\left(\tilde{K}_{0}, \tilde{K}_{1}, \tilde{K}_{2}\right)
$$

can have the spectrum

$$
E_{k, n}(\varphi, I)=\hbar \omega(n+k), n=0,1, \ldots ; k \in \mathbb{R}^{+} .
$$

A crucial point now is the following: the spectrum (4) is not just a special case of (19), but the situation is more subtle:

Let $|k, n\rangle, n=0,1, \ldots$ be an eigenvector of $\tilde{K}_{0}$ with eigenvalue (14):

$$
\tilde{K}_{0}|k, n\rangle=(n+k)|k, n\rangle, n=0,1, \ldots ; k>0
$$


then nevertheless

$$
H(Q, P)|k, n\rangle=\hbar \omega(n+1 / 2)|k, n\rangle,
$$

where now the operators $Q$ and $P$ are expressed as functions of the $\tilde{K}_{j}$ :

$$
Q=Q(\vec{K})=\frac{\lambda_{0}}{\sqrt{2}}\left(A^{\dagger}+A\right), \quad P=P(\vec{K})=\frac{i \hbar}{\sqrt{2} \lambda_{0}}\left(A^{\dagger}-A\right), \lambda_{0}=\sqrt{\frac{\hbar}{M \omega}},
$$

with

$$
A=\left(\tilde{K}_{0}+k\right)^{-1 / 2} \tilde{K}_{-}, \quad A^{\dagger}=\tilde{K}_{+}\left(\tilde{K}_{0}+k\right)^{-1 / 2}, \quad \tilde{K}_{ \pm}=\tilde{K}_{1} \pm i \tilde{K}_{2}
$$

and

$$
\left[A, A^{\dagger}\right]=\mathbf{1} .
$$

The non-linear relations (23) are an inversion of the known Holstein-Primakoff representation of the $\tilde{K}_{j}$ in terms of $A$ and $A^{\dagger}$ [27] as discussed in detail in Ref. [13].

The $k$-independent relation (24) holds in any irreducible unitary representation $D_{k}^{(+)}$and is a consequence of the commutation relations (13) which imply

$$
\tilde{K}_{+}|k, n\rangle=[(2 k+n)(n+1)]^{1 / 2}|k, n+1\rangle, \quad \tilde{K}_{-}|k, n\rangle=[(2 k+n-1) n]^{1 / 2}|k, n-1\rangle,
$$

so that for any $k$

$$
A^{\dagger}|k, n\rangle=\sqrt{n+1}|k, n+1\rangle, \quad A|k, n\rangle=\sqrt{n}|k, n-1\rangle .
$$

The Eqs. (22) and (23) are just the operator versions of the classical relations

$$
q(\varphi, I)=\sqrt{\frac{2}{M \omega}} \frac{h_{1}(\varphi, I)}{\sqrt{h_{0}(\varphi, I)}}, \quad p(\varphi, I)=\sqrt{2 M \omega} \frac{h_{2}(\varphi, I)}{\sqrt{h_{0}(\varphi, I)}} .
$$

For more details see below, here especially sec. 5 !

The gist of the argument for allowing a possible discrepancy between the spectra (20) and (21), to be discussed in detail later on, is that - due to the multi-valuedness of the angle $\varphi$ the quantum version (18) of the HO Hamilton function $H(\varphi, I)$ can have a richer spectrum than $H(Q, P)$ which always has the spectrum (4), even if it acts in a Hilbert space with a representation $D_{k}^{(+)}, k \neq 1 / 2$, for which $\tilde{K}_{0}$ has the spectrum (14)!

Phrased differently: The quantities $q$ and $p$ generate global translations on the phase space $\mathcal{S}_{q, p}$, i.e. no point is preferred, especially not the origin. This is different for the global action of the generators $h_{j}$ which leave the origin of $\mathcal{S}_{q, p}$ and the corresponding point $I=0$ in $\mathcal{S}_{\varphi, I}$ invariant. Thus, the operators $Q$ and $P$, generators of translations in momentum and position space, respectively, "erase" the topological substructure induced by the critical point $(q, p)=(0,0)$ (or $I=0)$. That point is, however "taken care of" by the operators $\tilde{K}_{j}$, generators of symplectic transformations in $(q, p)$-space, which leave the point $(q=0, p=0)$ fixed!

So it makes a difference as to the choice of the primary degrees of freedom, whether one starts with $q$ and $p$ and their topologically trivial phase space (7), or whether one starts with $\varphi$ and $I$ and their topologically non-trivial phase space (8). The latter leads to a "richer" quantum mechanics than that of the former which is unable to do justice to the non-trivial topology of (8) and therefore has to "ignore" the additional structure! Whether this additional topological fine structure has indeed been "implemented by nature" and can be observed in the laboratory - or is merely a coordinate singularity (see subsec. 2.1) - has, of course, to be found out by experiments. 


\subsection{Contents overview}

The paper is organized as follows:

Sec. 2 collects some properties of the classical HO, with emphasis on the singular character of the transformation (2) at $(q=0, p=0)$ and on the dynamical role of the "new" basic coordinates $\varphi$ and $I$, including the celebrated adiabatic properties of the action variable $I$ and its role for certain 1-dimensional integrable systems with bounded orbits.

Sec. 3 discusses properties of the symplectic transformation group $S p(2, \mathbb{R})$ acting on the phase space (7): As already mentioned above, that group transforms any two points of that space into each other, except for the point $(0,0)$ which is left fixed. The orbits of three independent 1-dimensional subgroups generate three vector fields which are globally Hamiltonian. The generating Hamiltonian functions of these vector fields are essentially the functions (9) (expressed in terms of the variables $q$ and $p$ ). The Poisson brackets of these Hamiltonian functions generate the Lie algebra $\mathfrak{s o}(1,2)=\mathfrak{s p}(2, \mathbb{R})$ of the groups $S O^{\uparrow}(1,2)$ and $S p(2, \mathbb{R})$. The quantized version of that Lie algebra belongs to irreducible unitary representations $D_{k}^{(+)}, k=1 / 4$ and $k=3 / 4$ of the so-called "metaplectic" group. These representations are implemented in the even and odd parity subspaces of the usual Hilbert space $L^{2}(\mathbb{R}, d q)$ of the HO.

Sec. 4 describes the action of the group $S O^{\uparrow}(1,2)=S p(2, \mathbb{R}) / \mathbb{Z}_{2}$ on the $(\varphi, I)$ - phase space (8) the points of which are "coordinized" by the functions (9). The action of the group is symplectic, transitive (i.e. any two points may be transformed into each other), effective (i.e. the only group element which leaves all points invariant is the unit element) and globally Hamiltonian, i.e. the functions (9) are the generating functions of the vector fields associated with three independent 1-dimensional transformation subgroups of $S O^{\uparrow}(1,2)$. So we have a completely satisfactory "canonical" structure on the phase space (9) based on the group $S O^{\uparrow}(1,2)$ and its infinitely many covering groups. This section prepares the ground for a group theoretical quantization [28-30] of the phase space (8) in terms of appropriate irreducible unitary representations of those groups which provide the associated quantum theories.

The central sec. 5 discusses the quantization of the phase space (8) in terms of the irreducible unitary representations of the positive discrete series $D_{k}^{(+)}$of the group $S O^{\uparrow}(1,2)$ and its infinitely many covering groups. The generator $\hbar \tilde{K}_{0}$ of the rotation subgroup is the quantized version of the action variable $I$ and the Hamilton function $H=\omega I$. Its most general spectrum is given by Eq. (14). In physics the corresponding Hamilton operator (18) generates time translations:

$$
U(t)=e^{-i H t / \hbar}, \quad H=\hbar \omega \tilde{K}_{0}
$$

This means that the (dimensionless) time variable $\tilde{t}=\omega t$ mathematically represents the angle $\varphi$. As $\tilde{t}$ in general does not stop at $\tilde{t}=2 \pi$, it "runs" through several or very many coverings. As $\tilde{K}_{0}=N+k \mathbf{1}$ we have

$$
U(\tilde{t}=2 \pi)=e^{-2 \pi i k} \mathbf{1} .
$$

This shows explicitly that for an $m$-fold covering with $k$ as in Eq. (15) we get

$$
U(\tilde{t}=2 \pi m)=\mathbf{1} .
$$

I already stressed above that in passing from the quantum theory of the Lie algebra $\mathfrak{s o}(1,2)$ to that of the Born-Dirac-Heisenberg-Jordan-Weyl Lie algebra one loses the "fine structure" 
associated with the Bargmann index $k$. This is a result the importance of which reaches probably far beyond the simple HO! It allows to avoid the celebrated Stone-von Neumann uniqueness theorem without violating it! The usual Heisenberg uncertainty relations for $Q$ and $P$ remain untouched, but there are new uncertainty relations as to the operators $\tilde{K}_{j}, j=0,1,2$ [13].

Sec. 6 discusses properties and possible applications of the three types of coherent states associated with the Lie algebra $\mathfrak{s o}(1,2)$ (Schrödinger-Glauber, Perelomov and Barut-Girardello) to the HO. The last two of these coherent states are very probably of similar importance for experiments in quantum optics as is already well-known for the Schrödinger-Glauber coherent states. A number of interesting physical expectation values and their dependence on the index $k$ are discussed as well as the possible experimental production of such states: The Perelomov ones have been generated in the laboratories in the form of squeezed states, the Barut-Girardello ones to the best of my knowledge not yet.

Sec. 7 describes several explicit examples of Hilbert spaces with irreducible unitary representations of the series $D_{k}^{(+)}$. It starts with the conventional HO for which $k=1 / 2$ represented in the Hardy space $H_{+}^{2}\left(S^{1}, \vartheta\right)$ on the unit circle $S^{1}$. That space has the scalar product

$$
\left(f_{2}, f_{1}\right)_{+}=\frac{1}{2 \pi} \int_{S^{1}} d \vartheta f_{2}^{*}(\vartheta) f_{1}(\vartheta),
$$

the basis

$$
e_{n}(\vartheta)=e^{i n \vartheta}, n=0,1,2, \cdots,
$$

and the HO Hamilton operator

$$
H=\hbar \omega \tilde{K}_{0}, \quad \tilde{K}_{0}=\frac{1}{i} \partial_{\vartheta}+1 / 2 .
$$

All the well-known physical properties of the usual quantized $\mathrm{HO}$ can be derived in this framework, and even some more, because now we have three different kinds of coherent states! The second part of that sec. deals with concrete Hilbert spaces where the index $k$ of the irreducible unitary representations can have any real value $>0$. One of these is the space $L^{2}\left(\mathbb{R}_{+}, d u\right)$ with its orthonormal basis of Laguerre's functions.

Sec. 8 briefly recalls the description of a quantized free electromagnetic field in a cavity as an infinite set of HOs and the disturbing quantitative problems one encounters for the total ground state energy when using the value (5) of a single oscillator. In the $(\varphi, I)$-framework one has instead $E_{k, n=0}=\hbar \omega k$, where $k>0$, in principal, can be arbitrarily small. This may shed new light on the notorious cosmological constant problem and the origin of the related dark energy [17-24].

If different electromagnetic modes have different $k$ by exposing them to external electric or magnetic fields, the electromagnetic "vacuum" can even acquire some sort of anomalous refractive strucure. This may lead (perhaps) to an understanding of the recently observed "dichroism" of the vacuum in a strong static magnetic field [31].

The sec. closes with a very speculative remark on the possibility of "dark" normal matter.

Sec. 9 recalls the effective HOs one has if a HO particle is charged and an additional external electric field is applied or if a free charged particle is in an external magnetic field. Here, too, one may introduce angle and action variables, the quantized versions of which may lead to a shift of the usual ground state levels.

Sec. 10 briefly discusses the (canonical) quantum statistics of a system with the energy levels (19), in order to see which thermodynamical quantities depend on $k$ and which not. 
Appendix A gives the technical details for the calculation of the action variables associated with the potentials discussed in subsec. 2.3. Appendix B summarizes some essential properties of the universal covering group of $S O^{\uparrow}(1,2)$, its irreducible unitary representations of the positive discrete series and those of the $m$-fold covering groups as special cases.

\subsection{Possible experiments}

The crucial question is, of course, whether there exist HOs in nature or may be prepared in the laboratory which have a spectrum of the type (19). It appears unnessary here to point out in detail the important implications this would have for the physics of many systems, not only for the HO!

For possible experimental setups one has to observe that the "primary observables" now are the operators $K_{j}, j=0,1,2$, with their algebraic structure (13), not as usual the position and momentum operators (22). Note also that $K_{0}$ is not the Hamiltonian, but $\omega K_{0}$, so that $E_{k n=0}(\varphi, I)$ from (19) can be the same for different $\omega$ and $k$ if their product is the same, i.e. the energy stays the same! One problem for the experiments is to find dynamical mechanisms which do not bring the usual $(q, p)$-dynamics into play, e.g. the dominant atomic dipole-transitions.

Following the original procedure of Mulliken and others [7] the value of $k$ in the spectrum (19) may, at least in principle, be determined as follows: According to Eqs. (11) and (2) the frequency $\omega$ of the oscillator can be changed either by changing its mass $M$ or by modifying the strength $b$ of the driving force. Let $\omega_{1}$ and $\omega_{2}$ be two known frequencies of the same system and let $E_{a}$ and $E_{b}$ two known fixed external energy levels different from the two ground state energies $E_{0}(j), j=1,2$, of the two slightly different versions of the same HO. If transitions

$$
E_{a} \rightarrow E_{0}(1)=\hbar \omega_{1} k, \quad E_{b} \rightarrow E_{0}(2)=\hbar \omega_{2} k
$$

with frequencies

$$
\omega_{a, 1}=\left[E_{a}-E_{0}(1)\right] / \hbar, \quad \omega_{b, 2}=\left[E_{b}-E_{0}(2)\right] / \hbar,
$$

are possible and measurable, then one can determine the value of $k$ from the difference

$$
\omega_{a, 1}-\omega_{b, 2}=\left(E_{a}-E_{b}\right) / \hbar-k\left(\omega_{1}-\omega_{2}\right) .
$$

In the case of the vibrating diatomic molecules Mulliken investigated the levels $E_{a}$ and $E_{b}$ where the vibrational ground states of a higher electronic level and the levels $E_{0}(j)$ were the vibrational ground states of a lower electronic level of the two respective isotopes for

which the two frequencies $\omega_{j}$ differ because the corresponding reduced masses $\mu$ in $\omega=\sqrt{b / \mu}$ differ [7].

Note also that for $k \neq 1 / 2$ all energy levels of the spectrum (19) are shifted compared to the usual ones (41).

More refined versions of Mulliken's experiments with diatomic molecules using modern experimental techniques should be possible and appear highly desirable! In order to "freeze" the $(q, p)$-degrees of freedom when looking for $(\varphi, I)$-properties one should probably go to extremely low temperatures, even below the ground state energies (5). Experiments with ultracold molecules have reached an impressive stage of refinement [32] and the use of Feshbach resonances [33] has led to fascinating experimental results for low lying vibrational bound state levels of bosonic pairs of atoms in ultra-cold BE-condensates [34]. 
Furthermore, modern experimental techniques have provided sophisticated 1-dimensional harmonic traps [35], for ions [36], atoms [37] and BE-condensates [38], for which the frequency $\omega$ from (2) can be tuned from outside, by changing the force strength $b$ electronically. Approximate 1-dimensional harmonic traps with ultra-cold BE-condensates mainly in the ground state (5) have been built [39], the ground state energy being determined by laser light Bragg reflections off the "untrapped" expanding cloud of BEC atoms. Thus, these impressive experiments appear to be associated with the $(q, p)$-model of the HO! Nevertheless, similar such setups may provide new possibilities for a search after the energy levels (19), again most likely at extremely low temperatures.

In sec. 6 it will be pointed out in detail that expectation values and transition probabilities involving Perelomov coherent states are proportional to the index $k$. As these states have already been generated experimentally for $k=1 / 2$, they may perhaps also be produced for other (lower) values of $k$.

Then there are possible vacuum birefringence and (or) dichroism effects of photons by strong external electric or magnetic fields as mentioned in sec. 8.

Sec. 9 discusses shifts in the HO ground states of charged particles in external electric or magnetic fields.

Sec. 10 finally mentions the plans for determining the ground state energy of the HO by means of the Josephson effect [40]!

\subsection{Generalizations}

Finally it should be remembered that the harmonic oscillator is, of course, not the only important integrable physical system which classically can be described by angle and action variables (e.g. the const./r potential, see Refs. [4] and [1-3] for more examples). Quantizing those systems group theoretically one has to distinguish between the cases $I \in \mathbb{R}^{+}$and $I \in \mathbb{R}$. The latter has to be quantized in terms of the irreducible unitary representations of the Euclidean group of the plane $E(2)$ and its covering groups. For details see Ref. [25].

One has, however, to observe the following: If the group $S O(2) \cong S^{1} \subset S O^{\uparrow}(1,2)$ becomes a non-trivial subgroup of a larger compact group (i.e. not just a direct abelian factor) its topological properties can change drastically: E.g., if one passes from $S O^{\uparrow}(1,2)$ to $S O(3)$ the universal covering group is now the double covering $S U(2)$. Going from $S O^{\uparrow}(1,2)$ to $S O^{\uparrow}(1,3)$ one has the universal double covering $S L(2, \mathbb{C})$.

If, on the other hand, one goes from $S O^{\uparrow}(1,2)$ to $S O^{\uparrow}(2,3)=S p(4, \mathbb{R}) / \mathbb{Z}_{2}$, where $S p(4, \mathbb{R})$ is the symplectic group in 4 dimensions, one again encounters the subgroup $S O(2) \cong S^{1}$ as a factor in the maximal compact subgroup $S O(2) \times S O(3)$ and and also a positive discrete series of irreducible unitary representations of the group $S p(4, \mathbb{R})$ and its infinitely many covering groups [41]. This is just another special case of symplectic groups $S p(2 n, \mathbb{R})$ in $2 n$ dimensions: They have dimension $2 n^{2}+n$, rank $n$ (i.e. a maximal abelian set of $n$ commuting Lie algebra generators), the maximal compact subgroup $U(n) \cong S U(n) \times U(1)$ (which has rank $n$, too) and (positive) discrete series of irreducible unitary representations [42], including those of their universal covering groups associated with the factor $U(1)$ (the group $S U(n)$ on the other hand is simply connected [43]). This should be of interest for the discussion of quantum mechanical properties of higher-dimensional symplectic systems [1-3, 29, 44, 45]. 


\subsection{Range of the paper}

As the topics of the present paper reach from experimental to mathematical physics I shall have missed many papers relevant to the subjects mentioned. I apologize to the experts and hope to do more justice to their work in the future. Many more related Refs. are contained in my paper [13] to which I shall refer frequently in the present one. An essential difference between this paper and Ref. [13] is the almost complete focus on the possible consequences of a consistent quantum mechanics for the angle-action variable description of the harmonic oscillator in different branches of physics, which is lacking in the previous paper.

\section{Some properties of the classical harmonic oscillator}

\subsection{The globally singular relationship between the canonical pairs $(q, p)$ and $(\varphi, I)$}

The transformation (2) is locally symplectic ("canonical"):

$$
d q \wedge d p=d \varphi \wedge d I, \quad \text { or } \quad \frac{\partial(q, p)}{\partial(\varphi, I)}=1
$$

As the angle $\varphi$ is dimensionless and for other reasons it is convenient to introduce dimensionless quantities by means of the unit of length $\lambda_{0}$ from Eqs. (22) and Planck's constant $\hbar$ and restore the physical dimensions when necessary:

$$
\begin{aligned}
\tilde{q} & =q / \lambda_{0}, \quad \lambda_{0}=\sqrt{\frac{\hbar}{M \omega}}, \\
\tilde{p} & =p \lambda_{0} / \hbar, \\
\tilde{H} & =H /(\hbar \omega)=\frac{1}{2}\left(\tilde{q}^{2}+\tilde{p}^{2}\right), \\
\tilde{I} & =I / \hbar=\tilde{H} \\
\tilde{h}_{j} & =h_{j} / \hbar, j=0,1,2, \\
\tilde{t} & =\omega t, \\
d q \wedge d p & =\hbar d \tilde{q} \wedge d \tilde{p}=\hbar d \varphi \wedge d \tilde{I},
\end{aligned}
$$

Now

$$
\tilde{q}=\sqrt{2 \tilde{I}} \cos \varphi, \quad \tilde{p}=-\sqrt{2 \tilde{I}} \sin \varphi
$$

As

$$
\tilde{p} d \tilde{q}=\tilde{I} d \varphi-d(\tilde{I} \cos \varphi \sin \varphi)
$$


we have locally the four equivalent generating functions

$$
\begin{aligned}
d F_{1}(\tilde{q}, \varphi) & =\tilde{I} d \varphi-\tilde{p} d \tilde{q}, \quad \partial_{\varphi} F_{1}=\tilde{I}, \quad \partial_{\tilde{q}} F_{1}=-\tilde{p} \\
F_{1}(\tilde{q}, \varphi) & =\frac{1}{2} \tilde{q}^{2} \tan \varphi \\
d F_{2}(\tilde{q}, \tilde{I}) & =\tilde{p} d \tilde{q}+\varphi d \tilde{I} \\
F_{2}(\tilde{q}, \tilde{I}) & =\tilde{I} \arccos [\tilde{q} /(\sqrt{2 \tilde{I}})] \pm \frac{1}{2} \tilde{q} \sqrt{2 \tilde{I}-\tilde{q}^{2}} \\
d F_{3}(\tilde{q}, \tilde{p}) & =-\sqrt{2 \tilde{I}} \sin \varphi d \tilde{q}+\sqrt{2 \tilde{I}} \cos \varphi d \tilde{p} \\
F_{3}(\tilde{q} \tilde{p}) & =\tilde{q} \tilde{p} \\
d F_{4}(\varphi, \tilde{I}) & =\frac{1}{2}\left(\tilde{q}^{2}-\tilde{p}^{2}\right) d \varphi-\frac{\tilde{q} \tilde{p}}{\tilde{q}^{2}+\tilde{p}^{2}} d \tilde{I} \\
F_{4}(\varphi, \tilde{I}) & =\tilde{I} \cos \varphi \sin \varphi
\end{aligned}
$$

On $\mathcal{S}_{\varphi, \tilde{I}}$ we have the (trivial) equations of motion

$$
\dot{\varphi}=\frac{\partial \tilde{H}}{\partial \tilde{I}}=\frac{\partial \tilde{I}}{\partial \tilde{I}}=1, \quad \dot{\tilde{I}}=-\frac{\partial \tilde{I}}{\partial \varphi}=0,
$$

with the solutions (orbits)

$$
\varphi(\tilde{t})=\tilde{t}+\varphi_{0}, \quad \tilde{I}=\text { const. }>0
$$

Inserted into the Eqs. (45) we get the usual orbits on $\mathcal{S}_{\tilde{q}, \tilde{p}}$, except for the trivial one $(\tilde{q}(\tilde{t}), \tilde{p}(\tilde{t})) \equiv(0,0) !$

That $(\tilde{q}, \tilde{p})=(0,0)$ or $\tilde{I}=0$ is a singular point of the otherwise symplectic transformation (45) can be seen in different ways:

- The action variable appears as $\sqrt{\tilde{I}}$, i.e. one has a branch point at $\tilde{I}=0$.

- If one introduces $\rho=\sqrt{\tilde{I}}$ then the functional determinant

$$
\frac{\partial(\tilde{q}, \tilde{p})}{\partial(\varphi, \rho)}=\rho
$$

becomes singular for $\rho=0$.

- The differential $d \tilde{H}(\tilde{q}, \tilde{p})=\tilde{q} d \tilde{q}+\tilde{p} d \tilde{p}$ has a critical point at $(\tilde{q}, \tilde{p})=(0,0)$.

- The differentials (47) - (50) of the generating functions $F_{j}$ become singular for $(\tilde{q}, \tilde{p})=$ $(0,0)$ or $\tilde{I}=0$.

So one has to delete the origin of the phase space $\mathcal{S}_{\tilde{q}, \tilde{p}}$ in order to map it in a one-toone manner onto $\mathcal{S}_{\varphi, \tilde{I}}$ and vice versa! But the punctured $(\tilde{q}, \tilde{p})$-plane is no longer simply connected and topologically non-trivial (its first homotopy group $\pi_{1}$ is $\mathbb{Z}$ ). This non-trivial topology also manifests itself in the multi-valuedness of the angle $\varphi$ which is mathematically represented by the unit circle $S^{1} \cong \mathbb{R} \bmod 2 \pi$. This unit circle constitutes the multiplyconnected "configuration space" of the phase space $\mathcal{S}_{\varphi, \tilde{I}}$. One of its here essential properties can be read off Eq. (52): 
In the course of time the periodical motion in both phase spaces (7) and (8) passes the position $\varphi_{0}$ a few or many times. In this way the configuration space $S^{1} \subset \mathcal{S}_{\varphi, \tilde{I}}$ gets unwrapped onto the real axis $\mathbb{R}$ or at least a part of it, here represented by the variable $\tilde{t}$. $\mathbb{R}$ constitutes the universal covering space of $S^{1}$. A very similar situation in which the same $S O(2) \cong S^{1}$ plays a corresponding role is discussed in Ref. [25]. The local character of the transformation (45) and its singularity at $(\tilde{q}=0, \tilde{p}=0)$ is emphasized in Thirring's textbook [3].

Note that physically the point $(\tilde{q}=0, \tilde{p}=0)$ is the ground state (equilibrium point) of the classical $(\tilde{q}, \tilde{p})$-description of the oscillator motion. In the case of the $(\varphi, \tilde{I})$-description the notion of an angle does not make sense any more for $\tilde{I}=0$. But $\tilde{I}$ may be arbitrarily small as long as it stays positive. As $H=\omega I$ one can have $H \rightarrow 0$ for $I>0$ by (formally) taking the limit $\omega \rightarrow 0$.

\subsection{A symplectic scale transformation}

The replacement

$$
\varphi \rightarrow \varphi_{\beta}=\varphi / \beta, \quad \tilde{I} \rightarrow \tilde{I}_{\beta}=\beta \tilde{I}, \quad \beta>0,
$$

is symplectic $\left(d \varphi_{\beta} \wedge d \tilde{I}_{\beta}=d \varphi \wedge d \tilde{I}\right)$. The transformation implies (cf. Eq. (52))

$$
\tilde{t} \rightarrow \tilde{t}_{\beta}=\tilde{t} / \beta \text {. }
$$

From

$$
\tilde{q}_{\beta}=\sqrt{2 \tilde{I}_{\beta}} \cos \varphi_{\beta}, \quad \tilde{p}_{\beta}=-\sqrt{2 \tilde{I}_{\beta}} \sin \varphi_{\beta}
$$

we get

$$
\tilde{H}_{\beta}=\frac{1}{2}\left(\tilde{p}_{\beta}^{2}+\tilde{q}_{\beta}^{2}\right)=\tilde{I}_{\beta}=\beta \tilde{I}=\beta \tilde{H}
$$

and

$$
\frac{d \varphi_{\beta}}{d \tilde{t}_{\beta}}=\frac{\partial \tilde{H}_{\beta}}{\partial \tilde{I}_{\beta}}=\frac{\partial \tilde{I}_{\beta}}{\partial \tilde{I}_{\beta}}=1, \quad \Rightarrow \varphi_{\beta}\left(\tilde{t}_{\beta}\right)=\tilde{t}_{\beta}+\varphi_{\beta}(0) .
$$

Inserting this $\varphi_{\beta}\left(\tilde{t}_{\beta}\right)$ into Eqs. (56) yields the $\tilde{t}_{\beta}$-dependence for the variables $\tilde{q}_{\beta}$, $\tilde{p}_{\beta}$, analogously to the $\tilde{t}$-dependence of the coordinates (45).

As $\tilde{t}=\omega t$ (cf. Eq. (43) ) the transformation of the original dimensionful quantities is ambiguous:

1. One can choose

$$
t \rightarrow t_{\beta}=t / \beta, \omega \rightarrow \omega
$$

This implies (cf. Eq. (2) )

$$
q \rightarrow q_{\beta}=\sqrt{\beta} q, \quad p \rightarrow p_{\beta}=\sqrt{\beta} p, \quad H \rightarrow H_{\beta}=\beta H=\omega I_{\beta} .
$$

2. A second possibility is

$$
t \rightarrow t, \omega \rightarrow \omega_{\beta}=\omega / \beta
$$

with

$$
q \rightarrow q_{\beta}=\beta q, \quad p \rightarrow p_{\beta}=p, \quad H \rightarrow H_{\beta}=H=\omega_{\beta} I_{\beta}
$$

Both possibilities are not symplectic as to $q$ and $p$.

Without further restrictions on the values of $\beta$ the transformation (54) presupposes the existence of covering spaces for $S^{1}$, because $\varphi / \beta$ may be outside a given interval, e.g. $(-\pi, \pi]$. 


\subsection{Going beyond the harmonic oscillator}

\subsubsection{Time-dependent perturbations}

If we perturb $\tilde{H}_{0}=\tilde{I}_{0}$ by a time-dependent term

$$
\tilde{H}_{1}=\epsilon \tilde{I}_{0} f(\tilde{t}) \ll \tilde{I}_{0},
$$

where $f(\tilde{t})$ is independent of $\varphi$ and $\tilde{I}_{0}$, then

$$
\dot{\varphi}=\partial_{\tilde{I}_{0}}\left(\tilde{H}_{0}+\tilde{H}_{1}\right)=1+\epsilon f(\tilde{t}), \dot{\tilde{I}}=-\partial_{\varphi}\left(\tilde{H}_{0}+\tilde{H}_{1}\right)=0,
$$

so that

$$
\varphi(\tilde{t})=\tilde{t}+\epsilon \int_{0}^{\tilde{t}} d \tau f(\tau)+\varphi_{0}, \quad \tilde{I}=\text { const. }
$$

Thus, only the time-dependence of $\varphi$ gets modified, but not that of $\tilde{I}=\tilde{I}_{0}$ !

The latter property is a special case of the famous adiabatic theorem of mechanics which says that "small and slow" perturbations of integrable systems leave the values of action variables unchanged [1-4]. This does, of course, not mean that the energy remains conserved! As to the important perturbation theory of integrable systems described by angle and action variables see the Refs. [1-4].

\subsubsection{Interactions proportional to $\tilde{h}_{1}$ or $\tilde{h}_{2}$}

On the phase space (7) the Hamilton functions $H(\tilde{q}, \tilde{p})$ depend on the basic variables $\tilde{q}$ and $\tilde{p}$, well beyond that of the HO. Similarly the Hamilton functions on (8) have to be expressed by the basic variables (9). Simple examples for interaction terms added to $\tilde{H}=\tilde{I}$ are the following ones:

$$
\tilde{H}=\tilde{h}_{0}+\gamma \tilde{h}_{1}=\tilde{I}+\gamma \tilde{I} \cos \varphi,|\gamma|<1 .
$$

The eqs. of motion

$$
\begin{aligned}
\dot{\varphi} & =\partial_{\tilde{I}} \tilde{H}=1+\gamma \cos \varphi, \\
\dot{\tilde{I}} & =-\partial_{\varphi} \tilde{H}=\gamma \tilde{I} \sin \varphi
\end{aligned}
$$

have the solutions [46]

$$
\begin{aligned}
\tan \left[\left(\varphi(\tilde{t})-\varphi_{0}\right) / 2\right] & =\sqrt{\frac{1+\gamma}{1-\gamma}} \tan \left[\sqrt{1-\gamma^{2}}\left(\tilde{t}-\tilde{t}_{0}\right) / 2\right] \\
\tilde{I}(\tilde{t}) & =\tilde{I}_{0}\left[1+\gamma \cos \left(\varphi(\tilde{t})-\varphi_{0}\right)\right]^{-1}
\end{aligned}
$$

If we replace $\tilde{h}_{1}$ in Eq. (66) by $\tilde{h}_{2}=-\tilde{I} \sin \varphi$, we get the solutions [47]

$$
\begin{aligned}
\tan \left[\left(\varphi(\tilde{t})-\varphi_{0}\right) / 2\right] & =\sqrt{1-\gamma^{2}}\left\{\tan \left[\sqrt{1-\gamma^{2}}\left(\tilde{t}-\tilde{t}_{0}\right) / 2\right]-\gamma\right\} . \\
\tilde{I}(\tilde{t}) & =\tilde{I}_{0}\left[1-\gamma \sin \left(\varphi(\tilde{t})-\varphi_{0}\right)\right]^{-1} .
\end{aligned}
$$

According to the definitions of Refs. $[1,2]$ the angle $\varphi(\tilde{t})$ is the "fast" variable here and the action variable $\tilde{I}(\tilde{t})$ the "slow" one. This language means to say that the perturbation $\gamma \tilde{I} \cos \varphi($ or $-\gamma \tilde{I} \sin \varphi)$ for small $|\gamma|$ merely leads to small oscillations of the action variable 
around its unperturbed value $\tilde{I}_{0}$. This can be read off the above solutions immediately for $|\gamma| \ll 1$. Closely related to this type of behaviour is the concept of averaging the $\varphi$-dependent part of the perturbation over a period $2 \pi$, an often powerful tool for estimating the influence of perturbations on integrable systems [1-4]. Such averaging is especially discussed in Ref. [2].

On the other hand, for $|\gamma| \rightarrow 1$ the action variables $\tilde{I}(\tilde{t})$ in Eqs. (170) and (72) fluctuate enormously ("resonances")!

\subsubsection{Morse and other "integrable" potentials}

I briefly discuss three well-known integrable systems [48] with potentials for which the Hamilton functions $H(\varphi, \tilde{I})$ are not just proportional to $\tilde{I}$ like in the case of the HO, but are quadratic in the action variable. This is so for the potentials

$$
\begin{aligned}
V_{M o}(q)= & V_{0}\left(e^{-a q}-1\right)^{2}, q \in \mathbb{R} ; a, V_{0}: \text { const. }>0, \\
& V_{M o}(q) \geq V_{M o}(q=0)=0, \\
V_{s M o}(q)= & V_{0}\left[1-1 / \cosh ^{2}(a q)\right]=V_{0} \tanh ^{2}(a q), \quad V_{s M o}(q) \geq V_{s M o}(q=0)=0, \\
& q \in \mathbb{R}, \quad V_{0}>0, \\
V_{P T}(q)= & V_{0} \tan ^{2}(a q), \quad a q \in(-\pi / 2, \pi / 2), \quad V_{0}>0, \quad V_{P T}(q=0)=0 .
\end{aligned}
$$

The first one was introduced by Morse [49] in order to describe radial vibrations of diatomic molecules $(q=r \geq 0)$ somewhat better than the HO does, the second one is a sort of symmetrized Morse potential [50] and the third one a slightly modified version of a potential discussed by Pöschl and Teller [51] in order to improve upon certain properties of the Morse potential. The potentials $V_{M o}$ and $V_{s M o}$ have bound states (periodic motions) for $0<E<V_{0}$, the potential $V_{P T}$ has only bound states, for all $E>0$.

For small $a q \ll 1$ the potentials reduce to the $\mathrm{HO}$ one:

$$
V_{M o}(q) \approx V_{s M o}(q) \approx V_{P T}(q) \approx \frac{1}{2} M \omega_{0}^{2} q^{2}, \quad \omega_{0}=a \sqrt{2 V_{0} / M}
$$

The "integrable" potential [52]

$$
V_{c}(q)=V_{0}[a q-1 /(a q)]^{2}, \quad q>0, \quad V_{c}(q) \geq V_{c}(q=1 / a)=0,
$$

provides an example for which the energy is a linear function of the action variable $I$, like for the HO. For $a q \ll 1$ we here have the HO approximation

$$
V_{c}(q) \approx \frac{1}{2} M \omega_{0}^{2}(q-1 / a)^{2}, \quad \omega_{0}=2 a \sqrt{2 V_{0} / M}
$$

For any potential $V(q)$ with periodic orbits on the phase space (7) the action variable is defined by the closed path integral

$$
2 \pi I(E)=\oint_{C(E)} d q p(q, E), \quad p(q, E)= \pm \sqrt{2 M}[E-V(q)]^{1 / 2}
$$

where the integration is to be taken clockwise along the closed path $C(E)$ determined by the energy equation

$$
\frac{1}{2 M} p^{2}+V(q)=E \text {. }
$$


The factor $2 \pi$ in the definition (179) is due to the convention which uses the circular frequency $\omega_{0}=2 \pi / T$ and not $\nu=1 / T$.

The integral (79) describes the area of the region the boundary of which is given by the closed curve $C(E)$.

If we now insert the relations (2) into the integral (79) we get the identity $2 \pi I(E)=2 \pi I$. This shows explicitly that the mapping (2) is independent of the potential chosen.

If $q_{-}<q_{+}$are the inner and outer turning points of the motion we can replace the closed path integral in Eq. (79) by

$$
2 \pi I(E)=2 \sqrt{2 M} \int_{q_{-}}^{q_{+}} d q[E-V(q)]^{1 / 2} .
$$

(Notice that $p d q=p \dot{q} d t>0$ on the path $C(E)$ in both, the upper and the lower $(q, p)$-halfplanes.)

As we have three free parameters now, $M, a$ and $V_{0}$, we do not have to use Planck's constant in order to introduce dimensionless quantities

$$
\tilde{q}=a q, \quad \tilde{p}=\frac{p}{\sqrt{M V_{0}}}, \quad \tilde{E}=E / V_{0}, \quad \tilde{I}=I \omega_{0} / V_{0}
$$

\section{Morse potential}

For the potential $V_{M}(q)$ the epression (81) now takes the form

$$
\pi \tilde{I}(\tilde{E})=2 \int_{\tilde{q}_{-}}^{\tilde{q}_{+}} d \tilde{q}\left[\tilde{E}-\left(e^{-\tilde{q}}-1\right)^{2}\right]^{1 / 2} .
$$

The integral can be solved explicitly (cf. Appendix A) and the result is

$$
\tilde{I}=2(1-\sqrt{1-\tilde{E}}), \quad \Rightarrow \quad \tilde{E}(\tilde{I})=\tilde{I}\left(1-\frac{1}{4} \tilde{I}\right) .
$$

The inequality $0<\tilde{E}<1$ implies for $\tilde{I}$

$$
0<\tilde{I}<2
$$

Restoring the physical dimensions we get the Hamilton function

$$
H_{M o}(I)=\omega_{0} I\left(1-\frac{\omega_{0} I}{4 V_{0}}\right) .
$$

It yields the eqs. of motion

$$
\dot{I}=0, \quad \dot{\varphi}=\omega_{0}-\frac{\omega_{0}^{2} I}{2 V_{0}},
$$

which can be integrated immediately.

In order to quantize the system as to its sector of bound states, we merely have to replace the action variable $I$ by the operator $\hbar \tilde{K}_{0}$ (cf. Eq. (18)). This leads to the Hamilton operator

$$
H_{M o}(\vec{K})=\hbar \omega_{0} \tilde{K}_{0}-\frac{\left(\hbar \omega_{0}\right)^{2}}{4 V_{0}} \tilde{K}_{0}^{2}
$$


which, according to Eq. (20), yields the spectrum

$$
E_{k, n}=\hbar \omega_{0}(n+k)\left[1-\frac{\hbar \omega_{0}}{4 V_{0}}(n+k)\right]
$$

which for $k=1 / 2$ is well-known [53]. Concrete Hilbert spaces and eigenfunctions are provided by irreducible unitary representations as discussed in sec. 7. The eigenfunctions of $H_{M o}(\vec{K})$ do not, of course, have to be solutions of the Schrödinger eq. in $q$-space, as is the case in Refs. [53]. But, because of the unitary equivalences, all physical predictions are the same!

As the square bracket in Eq. (89) should be positive one has to cut off the spectrum at a maximal $n=n_{\max }$, like it is done usually.

\section{The other potentials}

For the potential (74) one gets (cf. Appendix A) the same form for the Hamilton function as in Eq. (86), namely

$$
H_{s M o}(I)=\omega_{0} I\left(1-\frac{\omega_{0} I}{4 V_{0}}\right) .
$$

For the potential (75) one obtains (cf. Appendix A)

$$
\tilde{I}=2(\sqrt{\tilde{E}+1}-1), \quad \Rightarrow \quad H_{P T}(I)=\omega_{0} I\left(1+\frac{\omega_{0} I}{4 V_{0}}\right)
$$

which may be quantized accordingly. Again the result is well-known for $k=1 / 2$ [54].

Finally one obtains for the potential (77)

$$
H_{c}(I)=\omega_{0} I, \quad \omega_{0}=2 a \sqrt{2 V_{0} / M} .
$$

Comparison of $H_{s M o}(I)$ with $H_{M o}(I)$ and of $H_{c}(I)$ with $H_{H O}(I)$ shows that the possible orbits of motion may not depend on the details of the potentials $V(q)$, but only on some generic properties represented by the associated $H(I)$. There is still, however, the possibility that the quantized systems have different indices $k$. This is indeed the case for the solutions of the Schrödinger eqs. with the Hamiltonians $H_{M o}(Q, P)$ and $H_{s M o}(Q, P)$ [55].

\subsubsection{Free non-relativistic particle}

According to the second of the Eqs. (27) we can rewrite the Hamilton function

$$
H_{0}(q, p)=\frac{1}{2 M} p^{2}
$$

of a free particle as

$$
H_{0}(\vec{h})=\omega h_{2}^{2} / h_{0}, \quad \vec{h}=\left(h_{0}, h_{1}, h_{2}\right) .
$$

What is remarkable is that one needs an additional time scale - here provided by $\omega$ - in order to express $H_{0}$ in terms of the functions (9)! 


\section{Action of the symplectic group on the phase space $\mathcal{S}_{\tilde{q}, \tilde{p}}$}

The transformation group $S O^{\uparrow}(1,2)$ and its double covering, the symplectic group in 2 dimensions $S p(2, \mathbb{R})$, play a significant role in the following discussions. Some of their main properties have been summarized in Appendices A and B of Ref. [13]. In order to keep the present paper at least partially self-contained, some of those properties needed here are again sketched below (secs. $3-5$ ) and in Appendix B of this article.

The present Section provides a systematic justification for the choice of the basic coordinates (9) on the phase space (8) in terms of the symplectic transformation group $S p(2, \mathbb{R})$ on the phase space (7), without assuming this to be the phase space of the HO.

\subsection{Global and infinitesimal transformations, "observables"}

The elements of the symplectic group $G_{1} \equiv S p(2, \mathbb{R}) \quad(=S L(2, \mathbb{R}))$ are given by the matrices

$$
g_{1}=\left(\begin{array}{ll}
a_{11} & a_{12} \\
a_{21} & a_{22}
\end{array}\right), a_{j k} \in \mathbb{R}, \operatorname{det} g_{1}=1,
$$

which have the (defining) property

$$
g_{1}^{T} \cdot\left(\begin{array}{cc}
0 & 1 \\
-1 & 0
\end{array}\right) \cdot g_{1}=\left(\begin{array}{cc}
0 & 1 \\
-1 & 0
\end{array}\right) .
$$

If we introduce

$$
x=\left(\begin{array}{l}
\tilde{q} \\
\tilde{p}
\end{array}\right) \in \mathcal{S}_{\tilde{q}, \tilde{p}} \cong \mathbb{R}^{2},
$$

then the elements $g_{1}$ of $S p(2, \mathbb{R})$ act on $x$ as

$$
x \rightarrow x^{\prime}=g_{1} \cdot x, \quad g_{1} \in G_{1} \equiv S p(2, \mathbb{R}),
$$

with the property

$$
d \tilde{q}^{\prime} \wedge d \tilde{p}^{\prime}=d \tilde{q} \wedge d \tilde{p}
$$

i.e. the transformations (98) leave the symplectic form

$$
\omega_{\tilde{q}, \tilde{p}}=d \tilde{q} \wedge d \tilde{p}
$$

invariant.

The group action (98) has some other remarkable properties:

The whole group transforms the point $x=0$ into itself and acts transitively on the complement

$$
\mathcal{S}_{\tilde{q}, \tilde{p} ; 0} \equiv \mathcal{S}_{\tilde{q}, \tilde{p}}-\{x=0\} \cong \mathbb{R}^{2}-\{(0,0)\},
$$

i.e., if $x_{1}$ and $x_{2}$ are any two points of $\mathcal{S}_{\tilde{q}, \tilde{p} ; 0}$, then they can be transformed into each other by an element of $G_{1}$. This can easily be seen by considering the first two of the following 
1-parameter subgroups of $G_{1}$ :

$$
\begin{array}{ll}
R_{1}: & r_{1}=\left(\begin{array}{cc}
\cos (\theta / 2) & \sin (\theta / 2) \\
-\sin (\theta / 2) & \cos (\theta / 2)
\end{array}\right), \theta \in(-2 \pi,+2 \pi], \\
A_{1}: & a_{1}=\left(\begin{array}{cc}
e^{-\tau / 2} & 0 \\
0 & e^{\tau / 2}
\end{array}\right), \tau \in \mathbb{R}, \\
B_{1}: & b_{1}=\left(\begin{array}{cc}
\cosh (s / 2) & \sinh (s / 2) \\
\sinh (s / 2) & \cosh (s / 2)
\end{array}\right), s \in \mathbb{R} ; \\
N_{1}: & n_{1}=\left(\begin{array}{ll}
1 & \xi \\
0 & 1
\end{array}\right), \xi \in \mathbb{R} .
\end{array}
$$

Each element $g_{1}$ has a (Cartan) decomposition $g_{1}=k_{2} \cdot a_{1} \cdot k_{1}$ or $g_{1}=k_{2} \cdot b_{1} \cdot k_{1}$ and a unique (Iwasawa) decomposition $g_{1}=k_{1} \cdot a_{1} \cdot n_{1}$, where $k_{1}, k_{2} \in R_{1}$.

Now, let $x_{1}$ and $x_{2}$ be any two points of $\mathcal{S}_{\tilde{q}, \tilde{p} ; 0}$. First rotate $x_{1}$ by an element of $R_{1}$ into $x_{1}^{\prime}$, where $\tilde{p}_{1}^{\prime}=0$ and $\tilde{q}_{1}^{\prime}$ has the same sign as $\tilde{q}_{2}$. Then use an element of $A_{1}$ so that $e^{-\tau / 2} \tilde{q}_{1}^{\prime}=\tilde{q}_{1}^{\prime \prime}=\sqrt{\tilde{q}_{2}^{2}+\tilde{p}_{2}^{2}}$. Finally rotate the point $\left(\tilde{q}_{1}^{\prime \prime}, 0\right)$ into $x_{2}$.

The group $G_{1}$ acts also effectively on $\mathcal{S}_{q, p ; 0}$, that is to say, if

$$
x=g_{1} \cdot x \quad \forall x,
$$

then $g_{1}$ is the identity element

$$
e=E_{2} \equiv\left(\begin{array}{ll}
1 & 0 \\
0 & 1
\end{array}\right)
$$

\subsection{Vector fields and their associated Hamiltonian functions}

The 1-parameter subgroups (102) - (105) generate vectorfields on $\mathcal{S}_{q, p ; 0}$ in the following sense: Let $\Gamma=\{\gamma(s)\}$ be a 1-parameter group such that $\gamma(s=0)=1$ and let $f(x)$ be a smooth function. Then the $\Gamma$-associated vectorfield $\tilde{A}_{\Gamma}$ is defined by

$$
\left[\tilde{A}_{\Gamma} f\right](x)=\lim _{s \rightarrow 0} \frac{1}{s}[f(\gamma(-s) \cdot x)-f(x)] .
$$

From the first three subgroups above we get the following 3-dimensional basis of vectorfields associated with the group $G_{1}$ :

$$
\begin{aligned}
\tilde{A}_{R_{1}} & =\frac{1}{2}\left(\tilde{q} \partial_{\tilde{p}}-\tilde{p} \partial_{\tilde{q}}\right), \\
\tilde{A}_{A_{1}} & =\frac{1}{2}\left(\tilde{q} \partial_{\tilde{q}}-\tilde{p} \partial_{\tilde{p}}\right), \\
\tilde{A}_{B_{1}} & =-\frac{1}{2}\left(\tilde{p} \partial_{\tilde{q}}+\tilde{q} \partial_{\tilde{p}}\right),
\end{aligned}
$$

They obey the Lie algebra $\mathfrak{s p}(2, \mathbb{R})=\mathfrak{s o}(1,2)$ :

$$
\left[\tilde{A}_{R_{1}}, \tilde{A}_{A_{1}}\right]=\tilde{A}_{B_{1}}, \quad\left[\tilde{A}_{R_{1}}, \tilde{A}_{B_{1}}\right]=-\tilde{A}_{A_{1}}, \quad\left[\tilde{A}_{A_{1}}, \tilde{A}_{B_{1}}\right]=-\tilde{A}_{R_{1}} .
$$

Notice that the vector fields (109) - (111) vanish for $x=0$, a point to be excluded! 
These vector fields are global Hamiltonian ones, that is to say there exist global functions $\check{g}(x)$ on $\mathcal{S}_{q, p ; 0}$ such that the vector fields may be written as

$$
-\left[\partial_{\tilde{p}} \check{g}(x) \partial_{\tilde{q}}-\partial_{\tilde{q}} \check{g}(x) \partial_{\tilde{p}}\right]
$$

The three Hamiltonian functions here are

$$
\begin{array}{ll}
R_{1}: & \check{g}_{0}(x)=\frac{1}{4}\left(\tilde{q}^{2}+\tilde{p}^{2}\right), \\
A_{1}: & \check{g}_{2}(x)=-\frac{1}{2} \tilde{q} \tilde{p}, \\
B_{1}: & \check{g}_{1}(x)=\frac{1}{4}\left(-\tilde{q}^{2}+\tilde{p}^{2}\right) .
\end{array}
$$

(The numbering of the functions is mere convention.)

Their Poisson brackets obey the Lie algebra $\mathfrak{s p}(2, \mathbb{R})=\mathfrak{s o}(1,2)$, too:

$$
\left\{\check{g}_{0}, \check{g}_{1}\right\}_{\tilde{q}, \tilde{p}}=-\check{g}_{2}, \quad\left\{\check{g}_{0}, \check{g}_{2}\right\}_{\tilde{q}, \tilde{p}}=\check{g}_{1}, \quad\left\{\check{g}_{1}, \check{g}_{2}\right\}_{\tilde{q}, \tilde{p}}=\check{g}_{0}
$$

The squares of the $\check{g}_{j}(x)$ fulfill the relation

$$
\check{g}_{0}^{2}-\check{g}_{1}^{2}-\check{g}_{2}^{2}=0 \text {. }
$$

On the other hand, the vector fields induced by the following translations, but now on the phase space $\mathcal{S}_{\tilde{q}, \tilde{p}}$,

$$
\tilde{q} \rightarrow \tilde{q}+a, \tilde{p} \rightarrow \tilde{p} ; \tilde{q} \rightarrow \tilde{q}, \tilde{p} \rightarrow \tilde{p}-b ; a, b \in \mathbb{R}
$$

are

$$
\tilde{A}_{\tilde{q}}=-\partial_{\tilde{q}} ; \quad \tilde{A}_{\tilde{p}}=\partial_{\tilde{p}}
$$

with the Hamiltonian functions

$$
\check{g}_{\tilde{q}}(x)=\tilde{p}, \quad \check{g}_{\tilde{p}}(x)=\tilde{q},
$$

the Poisson brackets of which generate the usual Born-Dirac-Heisenberg-Jordan-Weyl (Lie) algebra (called BDHJW-algebra in the following ${ }^{1}$ ) with its basis $\{\tilde{q}, \tilde{p}, 1\}$ !

The bilinear functions (114) - (116) are the generators of the infinitesimal transformations associated with the transformations (98) of the subgroups (102) - (104):

$$
\begin{aligned}
\left\{\check{g}_{0}, \tilde{q}\right\} & =-\frac{1}{2} \tilde{p}, & \left\{\check{g}_{0}, \tilde{p}\right\} & =\frac{1}{2} \tilde{q}, \\
\left\{\check{g}_{1}, \tilde{q}\right\} & =-\frac{1}{2} \tilde{p}, & \left\{\check{g}_{1}, \tilde{p}\right\} & =-\frac{1}{2} \tilde{q}, \\
\left\{\check{g}_{2}, \tilde{q}\right\} & =\frac{1}{2} \tilde{q}, & \left\{\check{g}_{2}, \tilde{p}\right\} & =-\frac{1}{2} \tilde{p} .
\end{aligned}
$$

Integrated they give the transformations (98) of the subgroups (102) - (104), except for an unessential overall minus-sign of the group parameters, a consequence of the definition (113).

\footnotetext{
${ }^{1}$ The usual terminology is "Heisenberg-" or "Weyl-" algebra, but I think this to be unjust towards the contributions of the other authors.
} 
It is evident that the phase spaces (97) and (101) have not only quite different topological but, as a consequence, also essentially different canonical structures as to the transformation groups which act transitively on them: The phase space (97) has the translations (119) with their associated central extension (characterized by $\{\tilde{q}, \tilde{p}\}=1$ ) as its "canonical" group, but the phase space (101) the symplectic group $\operatorname{Sp}(2, \mathbb{R})$. This difference has important consequences for the quantum theory as we shall see!

The Hamiltonian functions (121) play a double role on the phase space $\mathcal{S}_{\tilde{q}, \tilde{p}}$ : They are the generators of the (canonical) translations and at the same time they are the basic classical "observables" on that phase space. Similarly one may consider the Hamiltonian functions (114) - (116) as basic observables on $\mathcal{S}_{\tilde{q}, \tilde{p} ; 0}$. However, there is the following ambiguity: Given a triple $\left(\check{g}_{0}>0, \check{g}_{1}, \check{g}_{2}\right)$ with the property (118), then the 2 pairs $(\tilde{q}, \tilde{p})$ and $(-\tilde{q},-\tilde{p})$ are compatible with a given triple. For further discussions of this important point see below.

The group $S p(2, \mathbb{R}$ ) is not only a transformation (automorphism) group of the BDHJWalgebra but the relations (117), (122) - (124) and $\{\tilde{q}, \tilde{p}\}=1$ show that the direct sum of the vector spaces of the Lie algebra $\mathfrak{s p}(2, \mathbb{R})$ and the BDHJW-algebra forms a 6-dimensional Lie algebra of its own. This feature plays a major role in the harmonic (Fourier) analysis of the BDHJW-group [56].

Whereas the coordinates of the points $x$ transform as vectors with respect to the group $S p(2, \mathbb{R}),($ cf. Eq. (98)), the Hamiltonian functions (114) - (116) transform as tensors of second degree: Applying the groups (102) - (104) to $\tilde{q}$ and $\tilde{p}$ and inserting the results into the r.h. sides of the expressions (114) - (116) yields the following transformations

$$
\begin{aligned}
& R_{1}: \quad \check{g}_{0}(x) \rightarrow \check{g}_{0}\left(x^{\prime}\right)=\check{g}_{0}(x), \\
& \check{g}_{1}(x) \rightarrow \check{g}_{1}\left(x^{\prime}\right)=\cos \theta \check{g}_{1}(x)+\sin \theta \check{g}_{2}(x), \\
& \check{g}_{2}(x) \rightarrow \check{g}_{2}\left(x^{\prime}\right)=-\sin \theta \check{g}_{1}(x)+\cos \theta \check{g}_{2}(x) \\
& A_{1}: \quad \check{g}_{0}(x) \rightarrow \check{g}_{0}\left(x^{\prime}\right)=\cosh \tau \check{g}_{0}(x)+\sinh \tau \check{g}_{1}(x), \\
& \check{g}_{1}(x) \rightarrow \check{g}_{1}\left(x^{\prime}\right)=\sinh \tau \check{g}_{0}(x)+\cosh \tau \check{g}_{1}(x), \\
& \check{g}_{2}(x) \rightarrow \check{g}_{2}\left(x^{\prime}\right)=\check{g}_{2}(x) ; \\
& B_{1}: \quad \check{g}_{0}(x) \rightarrow \check{g}_{0}\left(x^{\prime}\right)=\cosh s \check{g}_{0}(x)-\sinh s \check{g}_{2}(x), \\
& \check{g}_{1}(x) \rightarrow \check{g}_{1}\left(x^{\prime}\right)=\check{g}_{1}(x), \\
& \check{g}_{2}(x) \rightarrow \check{g}_{2}\left(x^{\prime}\right)=-\sinh s \check{g}_{0}(x)+\cosh s \check{g}_{2}(x) .
\end{aligned}
$$

These formulae show that the 3 functions $\check{g}_{j}$ transform as a 3 -vector with respect to the "Lorentz" group $S O^{\uparrow}(1,2)$ : The transformations (125) - (127) leave the quadratic form $\check{g}_{0}^{2}-$ $\breve{g}_{1}^{2}-\check{g}_{2}^{2}$ invariant. This is related to the fact that the group $S p(2, \mathbb{R})$ is a double covering of the group $S O^{\uparrow}(1,2)$ with the center $\mathbb{Z}_{2}=\{e,-e\}$ of $S p(2, \mathbb{R})$ as the kernel of the homomorphism $S p(2, \mathbb{R}) \rightarrow S O^{\uparrow}(1,2)$ (see Appendix B of Ref. [13]). Notice that the kernel (center) $\mathbb{Z}_{2}$ leaves the bilinear expressions (114) - (116) invariant.

\subsection{Space reflections and time reversal}

The center $\mathbb{Z}_{2}$ implements the parity operation

$$
\Pi: \quad \tilde{q} \rightarrow-\tilde{q}, \quad \tilde{p} \rightarrow-\tilde{p},
$$


which obviously is symplectic (it leaves the 2 -form $d \tilde{q} \wedge d \tilde{p}$ invariant).

More subtle is the implementation of the time reversal

$$
T: \quad \tilde{t} \rightarrow-\tilde{t}, \quad \tilde{q} \rightarrow \tilde{q}_{T}=\tilde{q}, \quad \tilde{p} \rightarrow \tilde{p}_{T}=-\tilde{p},
$$

which is not symplectic (we have $d \tilde{q} \wedge d \tilde{p} \rightarrow-d \tilde{q} \wedge d \tilde{p}$ ). However, this can be taken care of in analogy to quantum mechanics where time reversal - according to Wigner - is implemented by an anti-unitary transformation in Hilbert space:

$$
U_{T}: \quad \psi_{1} \rightarrow U_{T} \psi_{1}, \quad \psi_{2} \rightarrow U_{T} \psi_{2}, \quad\left(U_{T} \psi_{2}, U_{T} \psi_{1}\right)=\left(\psi_{1}, \psi_{2}\right)=\left(\psi_{2}, \psi_{1}\right)^{*},
$$

where $\left(\psi_{2}, \psi_{1}\right)$ denotes the complex-valued scalar product. As $\Im\left(\psi_{2}, \psi_{1}\right)$ defines a symplectic form [57] which changes sign under the complex conjugation (130), this suggests to change the order in $d \tilde{q} \wedge d \tilde{p}$ in the case of the time reversal (129):

$$
(d \tilde{q} \wedge d \tilde{p})_{T}=d \tilde{p}_{T} \wedge d \tilde{q}_{T}=-d \tilde{p} \wedge d \tilde{q}=d \tilde{q} \wedge d \tilde{p} .
$$

This has corresponding consequences for the associated Poisson brackets: Let $f^{(j)}(\tilde{q}, \tilde{p}), j=$ 1,2 , be two smooth functions on the phase space $\mathcal{S}_{\tilde{q}, \tilde{p}}$. With

$$
f_{T}^{(j)}(\tilde{q}, \tilde{p})=f^{(j)}(\tilde{q},-\tilde{p}), j=1,2,
$$

we define the time-reversed Poisson bracket by

$$
\left\{f^{(2)}, f^{(1)}\right\}_{T}=\left\{f_{T}^{(1)}, f_{T}^{(2)}\right\} .
$$

The definition is appropriate in the following sense: The time evolution of a function $f[\tilde{q}(\tilde{t}), \tilde{p}(\tilde{t})]$ (which does not depend explicitly on time) is given by

$$
\dot{f}=\{f, \tilde{H}\},
$$

where $\tilde{H}$ is the Hamilton function of the system. If $H_{T}(\tilde{q}, \tilde{p})=H(\tilde{q}, \tilde{p})$, we have for the timereversed Eq. (134)

$$
\frac{d f_{T}}{d(-\tilde{t})}=\{f, \tilde{H}\}_{T}=\left\{\tilde{H}, f_{T}\right\}, \quad \Rightarrow \dot{f}_{T}=\left\{f_{T}, \tilde{H}\right\}
$$

which is what one wants!

\subsection{The space $\mathcal{S}_{\tilde{q}, \tilde{p} ; 0}$ as a "homogeneous" one}

The phase space $\mathcal{S}_{\tilde{q}, \tilde{p} ; 0}$ can be interpreted as a homogeneous one as follows:

The subgroup (105) leaves the points of the line $\{(\tilde{q}, \tilde{p}=0)\}$ invariant, i.e. it is the "isotropy" or "little" group of these points. We have already seen that the group $S p(2, \mathbb{R})$ acts transitively on $\mathcal{S}_{\tilde{q}, \tilde{p} ; 0}$. Both properties imply that we can represent $\mathcal{S}_{\tilde{q}, \tilde{p} ; 0}$ as a homogeneous space, namely

$$
\mathcal{S}_{\tilde{q}, \tilde{p} ; 0} \cong S p(2, \mathbb{R}) / N_{1}
$$

i.e. the points $x \in \mathcal{S}_{\tilde{q}, \tilde{p} ; 0}$ are in one-to-one correspondence with the rest classes $g_{1} \cdot N_{1}$, where $g_{1} \in$ $S p(2, \mathbb{R})$. This is immediately plausible: The group $G_{1}=S p(2, \mathbb{R})$ has the unique (Iwasawa) subgroup decomposition $R_{1} \cdot A_{1} \cdot N_{1}$, with the topological product structure $S^{1} \times \mathbb{R}^{+} \times \mathbb{R}$. "Dividing out the subgroup $N_{1}$ " means dividing out the topological factor $\mathbb{R}$. The remaining product $S^{1} \times \mathbb{R}^{+}$corresponds to the polar coordinates of the punctured plane $\mathbb{R}^{2}-\{(0,0)\} \cong \mathcal{S}_{\tilde{q}, \tilde{p} ; 0}$. 


\subsection{Some quantum aspects}

The present subsec. is intended to illustrate the role the symplectic group $S p(2, \mathbb{R})$ from above plays in the conventional quantum mechanics of the HO, a role which remains unmentioned in the usual QM textbook discussions.

Let us apply the conventional quantization procedure to the functions (114) - (116) by replacing $\tilde{q}$ and $\tilde{p}$ by the operators $\tilde{Q}$ and $\tilde{P}$ and (Weyl) symmetrizing where necessary. We then get

$$
\begin{aligned}
& \check{g}_{0}(x) \rightarrow \frac{1}{4}\left(\tilde{P}^{2}+\tilde{Q}^{2}\right)=\tilde{K}_{0}, \\
& \check{g}_{1}(x) \rightarrow \frac{1}{4}\left(\tilde{P}^{2}-\tilde{Q}^{2}\right)=-\tilde{K}_{1} . \\
& \check{g}_{2}(x) \rightarrow-\frac{1}{4}(\tilde{Q} \tilde{P}+\tilde{P} \tilde{Q})=\tilde{K}_{2} .
\end{aligned}
$$

With

$$
\tilde{Q}=\frac{1}{\sqrt{2}}\left(a^{\dagger}+a\right), \quad \tilde{P}=\frac{i}{\sqrt{2}}\left(a^{\dagger}-a\right), \quad\left[a, a^{\dagger}\right]=\mathbf{1}
$$

we have

$$
\tilde{K}_{0}=\frac{1}{4}\left(2 a^{\dagger} a+1\right), \quad \tilde{K}_{1}=\frac{1}{4}\left(a^{\dagger^{2}}+a^{2}\right), \quad \tilde{K}_{2}=-\frac{i}{4}\left(a^{\dagger^{2}}-a^{2}\right)
$$

and

$$
\tilde{K}_{+}=\tilde{K}_{1}+i \tilde{K}_{2}=\frac{1}{2} a^{\dagger^{2}}, \tilde{K}_{-}=\tilde{K}_{1}-i \tilde{K}_{2}=\frac{1}{2} a^{2}
$$

The associated Lie algebra is

$$
\left[\tilde{K}_{0}, \tilde{K}_{1}\right]=i \tilde{K}_{2}, \quad\left[\tilde{K}_{0}, \tilde{K}_{2}\right]=-i \tilde{K}_{1}, \quad\left[\tilde{K}_{1}, \tilde{K}_{2}\right]=-i \tilde{K}_{0},
$$

or

$$
\left[\tilde{K}_{0}, \tilde{K}_{ \pm}\right]= \pm \tilde{K}_{ \pm}, \quad\left[\tilde{K}_{+}, \tilde{K}_{-}\right]=-2 \tilde{K}_{0}
$$

The relations (141) and (142) constitute a well-known realization of the Lie algebra $\mathfrak{s p}(2, \mathbb{R})=$ $\mathfrak{s o}(1,2)$ which yields two irreducible positive discrete series unitary representations of a twofold covering group of $S p(2, \mathbb{R})[58]$ :

Let $\left|n_{\text {osc }}\right\rangle$ be a number eigenstate of the harmonic oscillator Fock space:

$$
\begin{aligned}
a^{\dagger}\left|n_{o s c}\right\rangle=\sqrt{n_{o s c}+1}\left|n_{\text {osc }}+1\right\rangle, & & a\left|n_{\text {osc }}\right\rangle=\sqrt{n_{\text {osc }}}\left|n_{\text {osc }}-1\right\rangle, \\
a^{\dagger} a\left|n_{\text {osc }}\right\rangle=n_{\text {osc }}\left|n_{\text {osc }}\right\rangle, & & n_{\text {osc }}=0,1,2, \ldots
\end{aligned}
$$

As $\tilde{K}_{-}$annihilates the states $\left|n_{\text {osc }}=0\right\rangle$ and $\left|n_{\text {osc }}=1\right\rangle$,

$$
\tilde{K}_{-}\left|n_{o s c}=0\right\rangle=0, \quad \tilde{K}_{-}\left|n_{o s c}=1\right\rangle=0,
$$

we get two different irreducible unitary representations associated with the Lie algebra $\mathfrak{s p}(2, \mathbb{R})$ $=\mathfrak{s o}(1,2)$, one which is given by states with even numbers of Fock space quanta and one with odd numbers, both generated by the creation operator $\tilde{K}_{+}$: Because

$$
\tilde{K}_{0}\left|n_{o s c}\right\rangle=\frac{1}{2}\left(n_{o s c}+1 / 2\right)\left|n_{o s c}\right\rangle, n_{o s c}=0,1,2, \ldots
$$


we see that $\tilde{K}_{0}$ has the eigenvalues

$$
\left(2 n_{\text {osc }}+1 / 2\right) / 2=n+\frac{1}{4} \text { and }\left(2 n_{o s c}+1+1 / 2\right) / 2=n+\frac{3}{4}, n=0,1, \ldots,
$$

in the cases of even and odd numbers of quanta, respectively. That is to say, we get one irreducible unitary representation with $k=1 / 4$ and one with $k=3 / 4$.

As to the related groups these are true representations of a 2 -fold covering $M p(2, \mathbb{R})$ of $S p(2, \mathbb{R})=S L(2, \mathbb{R}) \cong S U(1,1)$ and a 4 -fold covering of $S O^{\uparrow}(1,2)$. These 2 -fold covering groups of the symplectic groups $S p(2 n, \mathbb{R})$ in $2 n$ dimensions are called "metaplectic" $[59,60]$ ones (for more details see below).

As the operators (137) - (139) commute with the parity transformation

$$
\Pi: \quad \tilde{Q} \rightarrow-\tilde{Q}, \quad \tilde{P} \rightarrow-\tilde{P}, \quad \Pi^{2}=\mathbf{1},
$$

the two irreducible representations may be associated with the eigenvalues \pm 1 of $\Pi$, respectively.

The two representations with $k=1 / 4$ and $k=3 / 4$ may, of course, be realized in the 2 subspaces $\mathcal{H}_{+}$and $\mathcal{H}_{-}$of the conventional Hilbert space $L^{2}(\mathbb{R}, d \tilde{q})$ of the harmonic oscillator with the orthonormal basis

$$
u_{n_{o s c}}(\tilde{q})=\frac{e^{-\tilde{q}^{2}}}{\sqrt{2^{n_{o s c}} \sqrt{\pi} n_{o s c} !}} H_{n_{o s c}}(\tilde{q}), \quad H_{n_{o s c}}(-\tilde{q})=(-1)^{n_{o s c}} H_{n_{o s c}}(\tilde{q})
$$

where $H_{n}(\tilde{q})$ is the $n$th Hermite polynomial.

The subspace $\mathcal{H}_{+}$for the unitary representation with $k=1 / 4$ is spanned by the Hermite functions with even Hermite polynomials $H_{n_{\text {osc }}}$ and the subspace $\mathcal{H}_{-}$for the representation with $k=3 / 4$ is spanned by the Hermite functions with odd Hermite polynomials.

In the "even" subspace $\mathcal{H}_{+}$the Hamiltonian

$$
\tilde{H}_{o s c}=2 \tilde{K}_{0}
$$

has the eigenvalues

$$
\left(n_{\text {osc }}+1 / 2\right), \quad n_{\text {osc }}=2 n, n=0,1,2, \ldots,
$$

and in the "odd" subspace $\mathcal{H}_{-}$its eigenvalues are

$$
\left(n_{\text {osc }}+1 / 2\right), \quad n_{\text {osc }}=2 n+1, n=0,1,2, \ldots
$$

Notice that the operators (140) map $\mathcal{H}_{+}$onto $\mathcal{H}_{-}$and vice versa!

The operators (23) for the two irreducible representations are

$$
A_{(1 / 4)}=\frac{1}{\sqrt{2}}\left(N_{\Phi}+1\right)^{-1 / 2} a^{2}, \quad A_{(1 / 4)}^{\dagger}=\frac{1}{\sqrt{2}} a^{\dagger^{2}}\left(N_{\Phi}+1\right)^{-1 / 2}, \quad N_{\Phi}=a^{\dagger} a,
$$

and

$$
A_{(3 / 4)}=\frac{1}{\sqrt{2}}\left(N_{\Phi}+2\right)^{-1 / 2} a^{2}, \quad A_{(3 / 4)}^{\dagger}=\frac{1}{\sqrt{2}} a^{\dagger^{2}}\left(N_{\Phi}+2\right)^{-1 / 2} .
$$

(The index $\Phi$ stands for "Fock".)

It follows from the properties of $a$ and $a^{\dagger}$ that

$$
A_{(1 / 4)}\left|n_{o s c}=2 n\right\rangle=\sqrt{n}|2 n-2\rangle, \quad A_{(1 / 4)}^{\dagger}\left|n_{o s c}=2 n\right\rangle=\sqrt{n+1}|2 n+2\rangle,
$$


and

$$
N_{(1 / 4)}=A_{(1 / 4)}^{\dagger} A_{(1 / 4)}=\frac{1}{2} N_{\Phi}, \quad\left[A_{(1 / 4)}, A_{(1 / 4)}^{\dagger}\right]=\mathbf{1} .
$$

Analogously we get

$$
A_{(3 / 4)}\left|n_{o s c}=2 n+1\right\rangle=\sqrt{n}|2 n-1\rangle, \quad A_{(3 / 4)}^{\dagger}\left|n_{o s c}=2 n+1\right\rangle=\sqrt{n+1}|2 n+3\rangle,
$$

and

$$
N_{(3 / 4)}=A_{(3 / 4)}^{\dagger} A_{(3 / 4)}=\frac{1}{2}\left(N_{\Phi}-\mathbf{1}\right), \quad\left[A_{(3 / 4)}, A_{(3 / 4)}^{\dagger}\right]=\mathbf{1}
$$

This means

$$
N_{1 / 4}\left|n_{\text {osc }}=2 n\right\rangle=n\left|n_{\text {osc }}=2 n\right\rangle, \quad N_{3 / 4}\left|n_{\text {osc }}=2 n+1\right\rangle=n\left|n_{\text {osc }}=2 n+1\right\rangle .
$$

According to Eqs. (22), (157) and (159) we may define in $\mathcal{H}_{+}$and $\mathcal{H}_{-}$the position and momentum operators

$$
\tilde{Q}_{(k)}=\frac{1}{\sqrt{2}}\left(A_{(k)}+A_{(k)}^{\dagger}\right), \quad \tilde{P}_{(k)}=\frac{i}{\sqrt{2}}\left(A_{(k)}^{\dagger}-A_{(k)}\right), \quad\left[\tilde{Q}_{(k)}, \tilde{P}_{(k)}\right]=i \mathbf{1}, \quad k=1 / 4,3 / 4
$$

The operators $\tilde{Q}_{(1 / 4)}$ and $\tilde{P}_{(1 / 4)}$ or $\tilde{Q}_{(3 / 4)}$ and $\tilde{P}_{(3 / 4)}$ have on the subspace $\mathcal{H}_{+}$or $\mathcal{H}_{-}$, respectively, the same matrix elements the operators (140) have on $\mathcal{H}=\mathcal{H}_{+} \oplus \mathcal{H}_{-}$! This is possible because in an infinite dimensional linear (Hilbert) space a genuine subspace may be isomorphic to the space itself. Here such a correspondence can be implemented by $\mathcal{H} \ni|n\rangle \leftrightarrow|2 n\rangle \in \mathcal{H}_{+}$ or $\mathcal{H} \ni|n\rangle \leftrightarrow|2 n+1\rangle \in \mathcal{H}_{-}$, respectively.

There is a crucial difference, however, between the "elementary" operators (140) and the "composite" ones (161): Using the general operator formula

$$
e^{C} B e^{-C}=B+[C, B]+\frac{1}{2 !}[C,[C, B]]+\frac{1}{3 !}[C,[C,[C, B]]]+\cdots,
$$

we get from Eqs. (140) and (141)

$$
U(\theta) a U(-\theta)=e^{i \theta / 2} a, \quad U(\theta) a^{\dagger} U(-\theta)=e^{-i \theta / 2} a^{\dagger}, \quad U(\theta)=e^{-i \tilde{K}_{0} \theta}, \quad \tilde{K}_{0}=\frac{1}{4}\left(2 a^{\dagger} a+1\right),
$$

so that

$$
U(\theta) \tilde{Q} U(-\theta)=\cos (\theta / 2) \tilde{Q}-\sin (\theta / 2) \tilde{P}, \quad U(\theta) \tilde{P} U(-\theta)=\sin (\theta / 2) \tilde{Q}+\cos (\theta / 2) \tilde{P} .
$$

Especially for $\theta=2 \pi$ we get the reflection

$$
U(\theta=2 \pi) \tilde{Q} U[-(\theta=2 \pi)]=-\tilde{Q}, \quad U(\theta=2 \pi) \tilde{P} U[-(\theta=2 \pi)]=-\tilde{P} .
$$

This shows that the operators (140) transform according to the subgroup (102) of $S p(2, \mathbb{R})$.

For $\theta=4 \pi$ the transformations (164) act as the identity on the pair $\tilde{Q}, \tilde{P}$, but we have

$$
U(\theta=4 \pi)=e^{-i 4 \pi\left(2 N_{o s c}+1\right) / 4}=e^{-i \pi} \mathbf{1}=-\mathbf{1}, \quad N_{o s c}=a^{\dagger} a .
$$

This shows again that $U(\theta), \theta \in[0,4 \pi)$, is not a true representation of the group $S p(2, \mathbb{R})$, but that it is one of its 2 -fold covering $M p(2, \mathbb{R})$ for which $U(\theta=8 \pi)=\mathbf{1}$. 
The reflections (165) may also be implemented by the simplified operator $\Pi$ :

$$
\Pi \tilde{Q} \Pi^{-1}=-\tilde{Q}, \quad \Pi \tilde{P} \Pi^{-1}=-\tilde{P}, \quad \Pi=e^{i \pi N_{o s c}},
$$

where the phase has been choosen such that

$$
\Pi\left|n_{\text {osc }}\right\rangle=(-1)^{n_{o s c}}\left|n_{o s c}\right\rangle .
$$

Contrary to the relations (163) we have on the other hand

$$
U(\theta) A_{(k)} U(-\theta)=e^{i \theta} A_{(k)}, \quad U(\theta) A_{(k)}^{\dagger} U(-\theta)=e^{-i \theta} A_{(k)}^{\dagger}, \quad U(\theta)=e^{-i \tilde{K}_{0} \theta},
$$

where $\tilde{K}_{0}$ is now given by $A_{(k)}^{\dagger} A_{(k)}+k \mathbf{1}$.

Thus, the operators (161) transform according to the group $S p(2, \mathbb{R}) / \mathbb{Z}_{2} \cong S O^{\uparrow}(1,2)$. This reflects the fact that the operators $\tilde{K}_{0}, \tilde{K}_{+}$and $\tilde{K}_{-}$transform according to the adjoint representation of $\operatorname{Sp}(2, \mathbb{R})[61]$.

The transformation properties of the operators (161) under the subgroups generated by the operators $\tilde{K}_{1}$ and $\tilde{K}_{2}$ are more complicated than those of Eqs. (140). For the latter we have, e.g.

$$
e^{-i \tau \tilde{K}_{2}} \tilde{Q} e^{i \tau \tilde{K}_{2}}=e^{\tau / 2} \tilde{Q}, \quad e^{-i \tau \tilde{K}_{2}} \tilde{P} e^{i \tau \tilde{K}_{2}}=e^{-\tau / 2} \tilde{P},
$$

where $\tilde{K}_{2}$ is given by Eq. (141). The transformation (170) is the usual "squeezing" transformation of quantum optics [62]. The corresponding transformation properties of the $\tilde{Q}_{(k)}$ and $\tilde{P}_{(k)}$ are more complicated (see sec. 4.4 below).

It is evident that by replacing the operators $a$ and $a^{\dagger}$ in Eqs. (141) and (142) by the operators $A_{(k)}$ and $A_{(k)}^{\dagger}$ of Eqs. (154) and (155) one may repeat the whole procedure indicated above, thereby splitting the subspaces $\mathcal{H}_{+}$and $\mathcal{H}_{-}$again into two subspaces and so on.

What is important for us at the present state of the discussion is that the quantized version of the " $(\tilde{q}, \tilde{p})$-model" of the HO carries two different irreducible unitary representations of a 2 -fold covering of the symplectic group $S p(2, \mathbb{R})$.

There is much more to come with the quantized version of the $(\varphi, \tilde{I})$-model of the HO:

\section{Action of the proper orthochronous homogeneous Lorentz group in $1+2$ dimensions on the phase space $\mathcal{S}_{\varphi, \tilde{I}}$}

\subsection{The basic canonical "observables" on $\mathcal{S}_{\varphi, \tilde{I}}$}

If we insert the relations

$$
\tilde{q}=\sqrt{2 \tilde{I}} \cos \varphi, \tilde{p}=-\sqrt{2 \tilde{I}} \sin \varphi,
$$

into the expressions (114) - (116) we get another set of functions $\check{h}_{j}(\varphi, \tilde{I}), j=0,1,2$, which again obey the Lie algebra $\mathfrak{s p}(2, \mathbb{R})=\mathfrak{s o}(1,2)$ with respect to the Poisson brackets (11):

$$
\begin{aligned}
\check{h}_{0}(\varphi, \tilde{I}) & =\frac{1}{2} \tilde{I}, \\
\check{h}_{1}(\varphi, \tilde{I}) & =-\frac{1}{2} \tilde{I} \cos (2 \varphi), \\
\check{h}_{2}(\varphi, \tilde{I}) & =\frac{1}{2} \tilde{I} \sin (2 \varphi),
\end{aligned}
$$


with

$$
\left\{\breve{h}_{0}, \check{h}_{1}\right\}_{\varphi, \tilde{I}}=-\check{h}_{2}, \quad\left\{\check{h}_{0}, \check{h}_{2}\right\}_{\varphi, \tilde{I}}=\check{h}_{1}, \quad\left\{\check{h}_{1}, \breve{h}_{2}\right\}_{\varphi, \tilde{I}}=\check{h}_{0} .
$$

This is not yet quite the form (9) we would like to have. But implementing the scaling (54) with $\beta=2$ yields the functions (9), except for the signs of $h_{1}$ and $h_{2}$ which may be reversed without affecting their properties and the Lie algebra structure (10).

Thus, we obtain on the phase space

$$
\mathcal{S}_{\varphi, \tilde{I}}=\{\sigma=(\varphi, \tilde{I}) ; \varphi \in \mathbb{R} \bmod 2 \pi, \tilde{I}>0\}
$$

the basic dimensionless functions

$$
\tilde{h}_{0}(\varphi, \tilde{I})=\tilde{I}>0, \quad \tilde{h}_{1}(\varphi, \tilde{I})=\tilde{I} \cos \varphi, \quad \tilde{h}_{2}(\varphi, \tilde{I})=-\tilde{I} \sin \varphi,
$$

which obey the Lie algebra

$$
\left\{\tilde{h}_{0}, \tilde{h}_{1}\right\}_{\varphi, \tilde{I}}=-\tilde{h}_{2}, \quad\left\{\tilde{h}_{0}, \tilde{h}_{2}\right\}_{\varphi, \tilde{I}}=\tilde{h}_{1}, \quad\left\{\tilde{h}_{1}, \tilde{h}_{2}\right\}_{\varphi, \tilde{I}}=\tilde{h}_{0} .
$$

The two obvious main reasons to pass from the functions (172) - (174) to the functions (177) are the following ones:

First, one would like $\tilde{h}_{0}$ to be equal to the Hamiltonian $\tilde{H}=\tilde{I}$ and, secondly, the basic periodic functions on $S^{1}$ are $\cos \varphi$ and $\sin \varphi$ from which all the higher ones, $\cos n \varphi, \sin n \varphi, n=$ $2,3, \ldots$, can be constructed. The functions $\cos 2 \varphi$ and $\sin 2 \varphi$ cannot serve that purpose! For related discussions of this point see Ref. [63].

A given triple $\left(\tilde{h}_{0}, \tilde{h}_{1}, \tilde{h}_{2}\right)$ with the property

$$
\tilde{h}_{0}^{2}-\tilde{h}_{1}^{2}-\tilde{h}_{2}^{2}=0, \quad \tilde{h}_{0}>0,
$$

determines a point $\sigma \in \mathcal{S}_{\varphi, \tilde{I}}$ uniquely. Eq. (179) shows that the phase space $\mathcal{S}_{\varphi, \tilde{I}}$ is diffeomorphic to a (light) cone with the tip deleted, i.e. it is topologically equivalent to $S^{1} \times \mathbb{R}^{+}$. Thus, $\mathcal{S}_{\varphi, \tilde{I}}$ has the same topological structure as $\mathcal{S}_{\tilde{q}, \tilde{p} ; 0}$ from above! It is, therefore, not surprising that the canonical group $S p(2, \mathbb{R})$ is intimately related to the coresponding one of $\mathcal{S}_{\varphi, \tilde{I}}$, namely the "proper orthochronous homogeneous Lorentz" group $S O^{\uparrow}(1,2) \cong S p(2, \mathbb{R}) / \mathbb{Z}_{2}$ which has the symplectic group $S p(2, \mathbb{R})$ as a double covering. $S O^{\uparrow}(1,2)$ is that connected subgroup of the four "pieces" of the group $O(1,2)$ which contains the unit element and is time-direction preserving [64]. More on this in subsec. 4.5 below.

The transformations of $S O^{\uparrow}(1,2)$ on $\mathcal{S}_{\varphi, \tilde{I}}$ are conveniently implemented by passing to the group $G_{0} \equiv S U(1,1)$ which is isomorphic to the group $S p(2, \mathbb{R})$ : The elements $g_{0} \in G_{0}$ are given by

$$
g_{0}=\left(\begin{array}{ll}
\alpha & \beta \\
\beta^{*} & \alpha^{*}
\end{array}\right), \quad \operatorname{det} g_{0}=|\alpha|^{2}-|\beta|^{2}=1 .
$$

They act on a 2-dimensional complex vector space $\mathbb{C}^{2}$ as

$$
g_{0} \cdot\left(\begin{array}{c}
z_{1} \\
z_{2}
\end{array}\right)=\left(\begin{array}{c}
z_{1}^{\prime} \\
z_{2}^{\prime}
\end{array}\right), \text { with }\left|z_{1}^{\prime}\right|^{2}-\left|z_{2}^{\prime}\right|^{2}=\left|z_{1}\right|^{2}-\left|z_{2}\right|^{2} .
$$

The isomorphism between the two groups $G_{0}$ and $G_{1}$ can be realized by the unitary matrix

$$
C_{0}=\frac{1}{\sqrt{2}}\left(\begin{array}{cc}
1 & -i \\
-i & 1
\end{array}\right), \operatorname{det} C_{0}=1, C_{0}^{-1}=\frac{1}{\sqrt{2}}\left(\begin{array}{cc}
1 & i \\
i & 1
\end{array}\right)=C_{0}^{\dagger},
$$


which yields

$$
C_{0} \cdot g_{1} \cdot C_{0}^{-1}=g_{0}
$$

The hermitian matrices

$$
\underline{\sigma}=\left(\begin{array}{cc}
\tilde{h}_{0}=\tilde{I} & \tilde{h}_{1}+i \tilde{h}_{2}=\tilde{I} e^{-i \varphi} \\
\tilde{h}_{1}-i \tilde{h}_{2}=\tilde{I} e^{i \varphi} & \tilde{h}_{0}=\tilde{I}
\end{array}\right), \operatorname{det} \underline{\sigma}=\tilde{h}_{0}^{2}-\tilde{h}_{1}^{2}-\tilde{h}_{2}^{2}=0,
$$

are in 1-1 correspondence to the points $\sigma \in \mathcal{S}_{\varphi, \tilde{I}}$. The transformations $\underline{\sigma} \rightarrow \underline{\sigma}^{\prime}$ under $S O^{\uparrow}(1,2)$ are implemented by

$$
\underline{\sigma} \rightarrow \underline{\sigma}^{\prime}=g_{0} \cdot \underline{\sigma} \cdot g_{0}^{\dagger}, \quad \operatorname{det} \underline{\sigma}^{\prime}=\operatorname{det} \underline{\sigma},
$$

where $g_{0}^{\dagger}$ denotes the hermitian conjugate of the matrix $g_{0}$.

The last equality in Eq. (185) follows from the property $\operatorname{det} g_{0}=\operatorname{det} g_{0}^{\dagger}=1$. Because $\operatorname{det} \underline{\sigma}=\tilde{h}_{0}^{2}-\tilde{h}_{1}^{2}-\tilde{h}_{2}^{2}$, the transformations (185) are indeed Lorentz transformations!

One sees immediately that $g_{0}$ and $-g_{0}$ lead to the same transformations of the 3-vectors $\left(\tilde{h}_{0}, \tilde{h}_{1}, \tilde{h}_{2}\right)$ and therefore of $\tilde{I}$ and $\varphi$. Thus, the group $S U(1,1)$ acts on the space $\mathcal{S}_{\varphi, \tilde{I}}$ only almost effectively with the kernel $Z_{2}$ representing the center of the twofold covering groups $S U(1,1)$ or $S p(2, \mathbb{R})$ of $S O^{\uparrow}(1,2)$. It is well-known that the latter group acts effectively and transitively on the forward light cone [65] and thus on $\mathcal{S}_{\varphi, \tilde{I}}$.

Applying a general $g_{0}$ to the matrix (184) yields the mapping:,

$$
\begin{aligned}
\sigma=(\varphi, \tilde{I}) & \rightarrow \sigma^{\prime}=\left(\varphi^{\prime}, \tilde{I}^{\prime}\right): \\
e^{i \varphi^{\prime}} & =\frac{\alpha^{*} e^{i \varphi}+\beta^{*}}{\alpha+e^{i \varphi} \beta} . \\
\tilde{I}^{\prime} & =\left|\alpha+e^{i \varphi} \beta\right|^{2} \tilde{I},
\end{aligned}
$$

As

$$
\frac{\partial \varphi^{\prime}}{\partial \varphi}=\left|\alpha+e^{i \varphi} \beta\right|^{-2}
$$

we have the equality

$$
d \varphi^{\prime} \wedge d \tilde{I}^{\prime}=d \varphi \wedge d \tilde{I}
$$

that is, the transformations (186) and (187) are symplectic.

It is, however, more instructive to look at the actions of 1-parameter subgroups of $S U(1,1)$ : The unitary transformation (182) maps the subgroups (102)-(105) of $G_{1}$ onto the following subgroups of $G_{0}$ :

$$
\begin{array}{ll}
R_{0}: & r_{0}=\left(\begin{array}{cc}
e^{i \theta / 2} & 0 \\
0 & e^{-i \theta / 2}
\end{array}\right), \theta \in(-2 \pi,+2 \pi], \\
A_{0}: & a_{0}=\left(\begin{array}{cc}
\cosh (\tau / 2) & i \sinh (\tau / 2) \\
-i \sinh (\tau / 2) & \cosh (\tau / 2)
\end{array}\right), \tau \in \mathbb{R}, \\
B_{0}: & b_{0}=\left(\begin{array}{cc}
\cosh (s / 2) & \sinh (s / 2) \\
\sinh (s / 2) & \cosh (s / 2)
\end{array}\right), s \in \mathbb{R}, \\
N_{0}: & n_{0}=\left(\begin{array}{cc}
1+i \xi / 2 & \xi / 2 \\
\xi / 2 & 1-i \xi / 2
\end{array}\right), \xi \in \mathbb{R} .
\end{array}
$$


(I here, too, list four - not independent - subgroups of $G_{0}$ because we shall need $N_{0}$ for the representation of $\mathcal{S}_{\varphi, \tilde{I}}$ as a homogeneous space below.) Their actions (185) on the 3-vector $\left(\tilde{h}_{0}, \tilde{h}_{1}, \tilde{h}_{2}\right)$ are given by

$$
\begin{aligned}
R_{0}: \quad \tilde{h}_{0} \rightarrow \tilde{h}_{0}^{\prime}=\tilde{h}_{0}, \\
\tilde{h}_{1} \rightarrow \tilde{h}_{1}^{\prime}=\cos \theta \tilde{h}_{1}-\sin \theta \tilde{h}_{2}, \\
\tilde{h}_{2} \rightarrow \tilde{h}_{2}^{\prime}=\sin \theta \tilde{h}_{1}+\cos \theta \tilde{h}_{2}, \\
A_{0}: \quad \tilde{h}_{0} \rightarrow \tilde{h}_{0}^{\prime}=\cosh \tau \tilde{h}_{0}+\sinh \tau \tilde{h}_{2}, \\
\tilde{h}_{1} \rightarrow \tilde{h}_{1}^{\prime}=\tilde{h}_{1}, \\
\tilde{h}_{2} \rightarrow \tilde{h}_{2}^{\prime}=\sinh \tau \tilde{h}_{0}+\cosh \tau \tilde{h}_{2}, \\
B_{0}: \quad \tilde{h}_{0} \rightarrow \tilde{h}_{0}^{\prime}=\cosh s \tilde{h}_{0}+\sinh s \tilde{h}_{1}, \\
\tilde{h}_{1} \rightarrow \tilde{h}_{1}^{\prime}=\sinh s \tilde{h}_{0}+\cosh s \tilde{h}_{1}, \\
\tilde{h}_{2} \rightarrow \tilde{h}_{2}^{\prime}=\tilde{h}_{2}, \\
N_{0}: \quad \tilde{h}_{0} \rightarrow \tilde{h}_{0}^{\prime}=\left(1+\xi \tilde{h}^{2} / 2\right) \tilde{h}_{0}+\xi \tilde{h}_{1}-\left(\xi^{2} / 2\right) \tilde{h}_{2}, \\
\tilde{h}_{1} \rightarrow \tilde{h}_{1}^{\prime}=\xi \tilde{h}_{0}+\tilde{h}_{1}-\xi \tilde{h}_{2}, \\
\tilde{h}_{2} \rightarrow \tilde{h}_{2}^{\prime}=\left(\xi^{2} / 2\right) \tilde{h}_{0}+\xi \tilde{h}_{1}+\left(1-\xi^{2} / 2\right) \tilde{h}_{2} .
\end{aligned}
$$

So we have rotations in the $\tilde{h}_{1}-\tilde{h}_{2}$ plane and two Lorentz "boosts", one in the $\tilde{h}_{0}-\tilde{h}_{2}$ plane and the other in the $\tilde{h}_{0}-\tilde{h}_{1}$ plane! All transformations leave the form $\tilde{h}_{0}^{2}-\tilde{h}_{1}^{2}-\tilde{h}_{2}^{2}$ invariant.

For the variables $\varphi$ and $\tilde{I}$ these transformations mean

$$
\begin{aligned}
R_{0}: \quad & \tilde{I}^{\prime}=\tilde{I}, \\
& e^{i \varphi^{\prime}}=e^{i(\varphi-\theta)}, \\
A_{0}: \quad & \tilde{I}^{\prime}=\rho_{a}(\tau, \varphi) \tilde{I}, \quad \rho_{a}(\tau, \varphi)=\cosh \tau-\sinh \tau \sin \varphi, \\
& \cos \varphi^{\prime}=\cos \varphi / \rho_{a}(\tau, \varphi), \\
& \sin \varphi^{\prime}=(\cosh \tau \sin \varphi-\sinh \tau) / \rho_{a}(\tau, \varphi), \\
B_{0}: \quad & \tilde{I}^{\prime}=\rho_{b}(s, \varphi) \tilde{I}, \rho_{b}(s, \varphi)=\cosh \tau+\sinh \tau \cos \varphi, \\
& \cos \varphi^{\prime}=(\cosh s \cos \varphi+\sinh s) / \rho_{b}(s, \varphi), \\
& \sin \varphi^{\prime}=\sin \varphi / \rho_{b}(s, \varphi), \\
& \tilde{I}^{\prime}=\rho_{n}(\xi, \varphi) \tilde{I}, \rho_{n}(\xi, \varphi)=1+\xi \cos \varphi+\xi^{2}(1+\sin \varphi) / 2, \\
& \cos \varphi^{\prime}=[\cos \varphi+\xi(1+\sin \varphi)] / \rho_{n}(\xi, \varphi), \\
& \sin \varphi^{\prime}=\left[\sin \varphi-\xi \cos \varphi-\xi^{2}(1+\sin \varphi) / 2\right] / \rho_{n}(\xi, \varphi) .
\end{aligned}
$$

As the center $\mathbb{Z}_{2}$ of $S U(1,1)$ acts as the identity in the transformations (194) - (201) the above transformation subgroups are actually those of $S O^{\uparrow}(1,2)=S U(1,1) / \mathbb{Z}_{2}$ which we shall denote by $R=R_{0} / \mathbb{Z}_{2}, A=A_{0} / \mathbb{Z}_{2}$ etc. in the following.

Transitivity of the $S O^{\uparrow}(1,2)$ group action on $\mathcal{S}_{\varphi, \tilde{I}}$ can be seen as follows: Any point $\sigma_{1}=$ $\left(\varphi_{1}, \tilde{I}_{1}\right)$ may be transformed into any other point $\sigma_{2}=\left(\varphi_{2}, \tilde{I}_{2}\right)$ : first transform $\left(\varphi_{1}, \tilde{I}_{1}\right)$ into $\left(0, \tilde{I}_{1}\right)$ by $r_{0}\left(\theta=\varphi_{1}\right)$, then map this point into $\left(\varphi_{0}=-\arctan \left(\sinh \tau_{0}\right), \tilde{I}_{2}\right)$ by $a_{0}\left(\tau_{0} ; \cosh \tau_{0}=\right.$ $\left.\tilde{I}_{2} / \tilde{I}_{1}\right)$ and finally transform $\left(\varphi_{0}, \tilde{I}_{2}\right)$ by $r_{0}\left(\theta=\varphi_{0}-\varphi_{2}\right)$ into $\sigma_{2}=\left(\varphi_{2}, \tilde{I}_{2}\right)$. 
For infinitesimal values of the parameters $\theta, \tau$ and $s$ the transformations (198) - (201) take the form

$$
\begin{aligned}
R: & \delta \varphi=-\theta,|\theta| \ll 1, \quad \delta \tilde{I}=0, \\
A: & \delta \varphi=-(\cos \varphi) \tau, \quad \delta \tilde{I}=-\tilde{I}(\sin \varphi) \tau, \quad|\tau| \ll 1, \\
B: & \delta \varphi=-(\sin \varphi) s, \quad \delta \tilde{I}=\tilde{I}(\cos \varphi) s, \quad|s| \ll 1 .
\end{aligned}
$$

According to Eq. (108) they induce on $\mathcal{S}_{\varphi, \tilde{I}}$ the vector fields

$$
\begin{aligned}
\tilde{A}_{R} & =\partial_{\varphi}, \\
\tilde{A}_{A} & =\cos \varphi \partial_{\varphi}+\tilde{I} \sin \varphi \partial_{\tilde{I}}, \\
\tilde{A}_{B} & =\sin \varphi \partial_{\varphi}-\tilde{I} \cos \varphi \partial_{\tilde{I}} .
\end{aligned}
$$

It is easy to check that the Lie algebra of these vector fields is isomorphic to the Lie algebra of $S O^{\uparrow}(1,2)$, and all its covering groups, of course.

The vector fields (205) - (207) are (global) Hamiltonian ones in the sense of Eq. (113). The corresponding Hamiltonian functions $f(\varphi, \tilde{I})$ are:

$$
\begin{aligned}
\tilde{A}_{R}: f_{R}(\varphi, \tilde{I}) & =-\tilde{I}, \\
\tilde{A}_{A}: f_{A}(\varphi, \tilde{I}) & =-\tilde{I} \cos \varphi, \\
\tilde{A}_{B}: f_{B}(\varphi, \tilde{I}) & =-\tilde{I} \sin \varphi .
\end{aligned}
$$

The Hamiltonian functions $f_{R}, f_{A}$ and $f_{B}$ obey the Lie algebra $\mathfrak{s o}(1,2)$ with respect to the Poisson brackets (11):

$$
\left\{f_{R}, f_{A}\right\}=-f_{B}, \quad\left\{f_{R}, f_{B}\right\}=f_{A}, \quad\left\{f_{A}, f_{B}\right\}=f_{R} .
$$

Reversing the minus signs on the right-hand side of Eqs. (208) and (209) we finally arrive again at our three basic classical observables introduced before:

$$
\tilde{h}_{0}(\varphi, \tilde{I}) \equiv-f_{R}=\tilde{I}, \quad \tilde{h}_{1}(\varphi, \tilde{I}) \equiv-f_{A}=\tilde{I} \cos \varphi, \quad \tilde{h}_{2}(\varphi, \tilde{I}) \equiv f_{B}=-\tilde{I} \sin \varphi .
$$

Thus, the canonical group $S O^{\uparrow}(1,2)$ of the symplectic space $\mathcal{S}_{\varphi, \tilde{I}}$ determines the basic "observables" (177) of that classical space.

\section{$4.2 \mathcal{S}_{\varphi, \tilde{I}}$ as a homogeneous space}

The transformation formulae (201) show that the subgroup $N_{0}$ leaves the half-line $\varphi=$ $-\pi / 2, \tilde{I}>0$, pointwise invariant, that is, $N_{0}$ is the stability group of those points. This implies that the symplectic space $\mathcal{S}_{\varphi, \tilde{I}}$ is diffeomorphic to the coset space $S O^{\uparrow}(1,2) / N_{0}$ :

$$
\mathcal{S}_{\varphi, \tilde{I}} \cong S O^{\uparrow}(1,2) / N_{0}
$$

Notice that the subgroups $N_{0}$ and $A_{0}$ do not contain the second center element $-e$ of $S U(1,1)$. The center $\mathbb{Z}_{2}$ is a subgroup of $R_{0}$.

In the language of the $\tilde{h}_{j}$ the transformations (197) leave the points $\left(\tilde{I}, \tilde{h}_{1}=0, \tilde{h}_{2}=\tilde{I}\right)$ invariant. 


\subsection{On the relationship between the phase spaces $\mathcal{S}_{\tilde{q}, \tilde{p} ; 0}$ and $\mathcal{S}_{\varphi, \tilde{I}}$}

The two phase spaces $\mathcal{S}_{\tilde{q}, \tilde{p} ; 0}$ and $\mathcal{S}_{\varphi, \tilde{I}}$ have the same topological structure $S^{1} \times \mathbb{R}^{+}$, but their correspondence is nevertheless not one-to-one. The term "topological" is somewhat imprecise here: the positive real numbers $\mathbb{R}^{+}$can be mapped in a one-to-one fashion onto the full real line $\mathbb{R}$ by $\tilde{I}=e^{a}, a=\ln \tilde{I}, \tilde{I} \in \mathbb{R}^{+}, a \in \mathbb{R}$, . This mapping is, however, not symplectic because $d \varphi \wedge d \tilde{I}=e^{a} d \varphi \wedge d a$. But as we are interested in preserving the symplectic structures, we consider only (usually local) "symplectomorphisms" [44].

In order to see the essential difference between the spaces $\mathcal{S}_{\tilde{q}, \tilde{p} ; 0}$ and $\mathcal{S}_{\varphi, \tilde{I}}$ let us look at the orbits of the transformation group $R_{1}$ (cf. Eq. (102)) on the former (cf. Eq. (98)) and those of $R_{0}$ (cf. Eq. (190)) on the latter (cf. Eqs. (194)):

If we start with $\theta=0$ and increase $\theta$ to $\pi$ then - according to Eq. (102) - the positive $\tilde{q}$-axis is rotated by $90^{\circ}$ onto the positive $\tilde{p}$-axis and the latter is rotated onto the negative $\tilde{p}$-axis. On the other hand - according to Eq. (194) - the $\tilde{h}_{1}$ - and $\tilde{h}_{2}$-axis are both rotated by $180^{\circ}$, i.e. they change sign. For $\theta=2 \pi$ the transformation (194) becomes the identity, whereas now the transformation (102) changes the sign of $x=(\tilde{q}, \tilde{p})^{T}$ ("T" here means "transpose"): $x \rightarrow-x$. Finally, for $\theta=4 \pi$ both groups act as the identity.

All this is, of course, a consequence of the fact that the effective transformation group on $\mathcal{S}_{\tilde{q}, \tilde{p} ; 0}$ is $S p(2, \mathbb{R})$, whereas the corresponding transformation group on $\mathcal{S}_{\varphi, \tilde{I}}$ is $S O^{\uparrow}(1,2)=$ $S p(2, \mathbb{R}) / \mathbb{Z}_{2}$ ! The situation is completely similar to the well-known transformations of the group $S U(2)$ on a 2-dimensional (complex spinor) vector space which induces a corresponding transformation of the group $S O(3)=S U(2) / \mathbb{Z}_{2}$ on a 3 -dimensional vector space. Here, too, a given element of $S O(3)$ corresponds to two elements $\pm u \in S U(2)$ ! In our case the $x \in \mathcal{S}_{\tilde{q}, \tilde{p} ; 0}$ are the "spinors" and the $\sigma \in \mathcal{S}_{\varphi, \tilde{I}}$ are the "vectors".

The remarks show in which way the two spaces $\mathcal{S}_{\tilde{q}, \tilde{p} ; 0}$ and $\mathcal{S}_{\varphi, \tilde{I}}$ differ globally despite the local equality $d \varphi \wedge d \tilde{I}=d \tilde{q} \wedge d \tilde{p}$ which is obviously invariant under the center $\mathbb{Z}_{2}$ (it sends $x$ to $-x$ and $\sigma$ to $\sigma$ ).

One may characterize the situation also in the following way [66]: If we identify the points $x$ and $-x$ on $\mathcal{S}_{\tilde{q}, \tilde{p} ; 0}$ then the group $S p(2, \mathbb{R})$ acts on this quotient space in the same way as the group $S O^{\uparrow}(1,2)$ on $\mathcal{S}_{\varphi, \tilde{I}}$ and we have the correspondence

$$
\mathcal{S}_{\varphi, \tilde{I}} \cong \mathcal{S}_{\tilde{q}, \tilde{p} ; 0} / \mathbb{Z}_{2}
$$

which follows also from comparing the homogeneous spaces (136) and (213).

A quotient space like (214) is called an "orbifold". An orbifold may be generated from a manifold M by identifying points which are connected by a finite discontinuous group $D_{n}$ of $n$ elements so that the orbifold is given by the quotient space $\mathrm{M} / D_{n}$. An orbifold generally has additional singularities as compared to the manifold from which it is constructed, as we shall see now:

In our case the orbifold $\mathcal{S}_{\tilde{q}, \tilde{p} ; 0} / \mathbb{Z}_{2}$ is a cone: Take the lower half of the $(\tilde{q}, \tilde{p})$-plane and rotate it around the $\tilde{q}$-axis till it coincides with the upper half of the plane such that the negative $\tilde{p}$-axis lies on the positive one. Then rotate the left half of the upper half plane around the positive $\tilde{p}$-axis till the negative $\tilde{q}$-axis coincides with the positive one. Finally glue the two $\tilde{q}$-half-axis together. The resulting space is a cone with its "tip" (vertex) at $x=0$. The tip constitutes a singularity to be deleted. It is a fixed point of the action of $\mathbb{Z}_{2}$. We thus arrive at the cone structure of the symplectic space (214) by a different route. 


\subsection{Relationships between the coordinates $\tilde{q}, \tilde{p}$ and $\tilde{h}_{0}, \tilde{h}_{1}, \tilde{h}_{2}$}

Further below we shall encounter several important relations between the quantum operator versions $\tilde{Q}, \tilde{P}, \tilde{K}_{0}, \tilde{K}_{1}$ and $\tilde{K}_{2}$ of the corresponding classical basic quantities $\tilde{q}, \tilde{p}, \tilde{h}_{0}, \tilde{h}_{1}$ and $\tilde{h}_{2}$. So it is useful to list a number of relations between the latter:

$$
\begin{aligned}
\tilde{q}(\varphi, \tilde{I})= & \sqrt{2 \tilde{I}} \cos \varphi=\sqrt{\frac{2}{\tilde{h}_{0}}} \tilde{h}_{1} ; \quad \tilde{h}_{0}=\tilde{I}, \quad \tilde{h}_{1}=\tilde{I} \cos \varphi, \\
\tilde{p}(\varphi, \tilde{I})= & -\sqrt{2 \tilde{I}} \sin \varphi=\sqrt{\frac{2}{\tilde{h}_{0}}} \tilde{h}_{2} ; \quad \tilde{h}_{2}=-\tilde{I} \sin \varphi, \\
\alpha= & \frac{1}{\sqrt{2}}(\tilde{q}+i \tilde{p})=\sqrt{\tilde{I}} e^{-i \varphi}=\tilde{h}_{+} / \sqrt{\tilde{h}_{0}}, \\
& \tilde{h}_{+}=\tilde{h}_{1}+i \tilde{h}_{2}=\tilde{I} e^{-i \varphi}, \tilde{h}_{-}=\tilde{h}_{1}-i \tilde{h}_{2}=\tilde{I} e^{i \varphi} .
\end{aligned}
$$

Also of interest are a number of Poisson brackets:

$$
\begin{aligned}
\left\{\tilde{h}_{0}, \tilde{q}\right\}_{\varphi, \tilde{I}} & =-\tilde{p}, \\
\left\{\tilde{h}_{0}, \tilde{p}\right\}_{\varphi, \tilde{I}} & =\tilde{q}, \\
\left\{\tilde{h}_{0}, \alpha\right\}_{\varphi, \tilde{I}} & =i \alpha .
\end{aligned}
$$

These are just the canonical eqs. of motion for the HO.

More complicated are the following Poisson brackets

$$
\begin{aligned}
\left\{\tilde{h}_{1}, \tilde{q}\right\}_{\varphi, \tilde{I}} & =-\frac{1}{2} \sin \varphi \tilde{q}-\cos \varphi \tilde{p}, \\
\left\{\tilde{h}_{1}, \tilde{p}\right\}_{\varphi, \tilde{I}} & =-\frac{1}{2} \sin \varphi \tilde{p}+\cos \varphi \tilde{q}, \\
\left\{\tilde{h}_{2}, \tilde{q}\right\}_{\varphi, \tilde{I}} & =-\frac{1}{2} \cos \varphi \tilde{q}+\sin \varphi \tilde{p}, \\
\left\{\tilde{h}_{2}, \tilde{p}\right\}_{\varphi, \tilde{I}} & =-\frac{1}{2} \cos \varphi \tilde{p}-\sin \varphi \tilde{q},
\end{aligned}
$$

where the right-hand sides may be expressed in different ways by using the quantities defined in Eqs. (215) - (218). Examples are

$$
\begin{aligned}
\left\{\tilde{h}_{+}, \alpha\right\}_{\varphi, \tilde{I}} & =\frac{1}{\sqrt{\tilde{I}}}\left(-\frac{1}{2} \alpha^{*}+i \alpha\right) \alpha=-\frac{1}{2} \sqrt{\tilde{I}}+\frac{i}{\tilde{I}^{3 / 2}}\left(\tilde{h}_{+}\right)^{2}, \\
\left\{\tilde{h}_{-}, \alpha\right\}_{\varphi, \tilde{I}} & =\frac{1}{\sqrt{\tilde{I}}}\left(-\frac{1}{2} \alpha+i \alpha^{*}\right) \alpha=\frac{i}{2} \sqrt{\tilde{I}}-\frac{1}{2 \tilde{I}^{3 / 2}}\left(\tilde{h}_{-}\right)^{2} .
\end{aligned}
$$

The brackets $\left\{\tilde{h}_{+}, \alpha^{*}\right\}_{\varphi, \tilde{I}}$ and $\left\{\tilde{h}_{-}, \alpha^{*}\right\}_{\varphi, \tilde{I}}$ follow from complex conjugation of the relations (228) and (227).

\subsection{Space reflections and time reversal}

The space reflections (128) may be implemented on $\mathcal{S}_{\varphi, \tilde{I}}$ by

$$
\Pi: \varphi \rightarrow \varphi \pm \pi, \quad \tilde{I} \rightarrow \tilde{I} .
$$


The reflection $\Pi$ leaves the symplectic form $d \varphi \wedge d \tilde{I}$ invariant (locally) and implies

$$
\Pi: \quad \tilde{h}_{0} \rightarrow \tilde{h}_{0}, \quad \tilde{h}_{1} \rightarrow-\tilde{h}_{1}, \quad \tilde{h}_{2} \rightarrow-\tilde{h}_{2} .
$$

The time reversal (129) can be implemented by

$$
T: \tilde{t} \rightarrow-\tilde{t}, \quad \varphi \rightarrow-\varphi, \quad \tilde{I} \rightarrow \tilde{I} .
$$

In order to make this transformation into a symplectic one, we also have to change the order of the factors in $d \varphi \wedge d \tilde{I}$ as discussed after Eq. (129) above. We now have

$$
T: \quad \tilde{h}_{0} \rightarrow \tilde{h}_{0}, \quad \tilde{h}_{1} \rightarrow \tilde{h}_{1}, \quad \tilde{h}_{2} \rightarrow-\tilde{h}_{2} .
$$

Notice that the space reflection properties (230) of the $\tilde{h}_{j}$ are different from those of the $\check{h}_{j}$ of Eqs. (172) - (174). The T-reversal properties are the same.

The relationship of the above $\Pi$ - and $T$-transformations to the different "pieces" of the homogeneous Lorentz group $O(1,2)$ is as follows: It follows from

$$
\tilde{h}_{j} \rightarrow \tilde{h}_{j}^{\prime}=\sum_{k=0}^{2} \Lambda_{j}^{k} \tilde{h}_{k},\left(\tilde{h}_{0}^{\prime}\right)^{2}-\left(\tilde{h}_{1}^{\prime}\right)^{2}-\left(\tilde{h}_{2}^{\prime}\right)^{2}=\left(\tilde{h}_{0}\right)^{2}-\left(\tilde{h}_{1}\right)^{2}-\left(\tilde{h}_{2}\right)^{2},
$$

that

$$
\operatorname{det}\left(\Lambda_{j}^{k}\right)= \pm 1, \quad \operatorname{sgn} \Lambda_{0}^{0}= \pm 1 .
$$

The group $S O^{\uparrow}(1,2)$ which contains the identity transformation is characterized by $\operatorname{det}\left(\Lambda_{j}^{k}\right)=$ $1, \operatorname{sgn} \Lambda_{0}^{0}=1$. The above transformations $\Pi$ and $T$ have both $\operatorname{sgn} \Lambda_{0}^{0}=1, \operatorname{but} \operatorname{det}\left(\Lambda_{j}^{k}\right)=1$ and $\operatorname{det}\left(\Lambda_{j}^{k}\right)=-1$, respectively.

\section{Quantizing the angle - action variables phase space $\mathcal{S}_{\varphi, \tilde{\mathbf{I}}}$ of the harmonic oscillator}

\subsection{Lie algebra of the self-adjoint observables $\tilde{K}_{j}$ and the structure of their irreducible representations}

The quantum theory of the HO described on the phase space $\mathcal{S}_{\tilde{q}, \tilde{p}}$ is a settled affair, due to the Stone-von Neumann uniqueness theorem for the irreducible unitary representations of the BDHJW-group [67]!

The situation is different, however, for the quantum theory of the HO described by the phase space $\mathcal{S}_{\varphi, \tilde{I}}$ of its angle and action variables. We have seen that the "canonical" group of that phase space is the group $S O^{\uparrow}(1,2)$ which has an infinite number of covering groups, due to its maximal compact rotation subgroup $S O(2)$. The group $S O^{\uparrow}(1,2)$ - and its covering groups has 3 classes of irreducible unitary representations [68]: the "principal", the "supplementary" or "complementary" series and two "discrete" series. In the principal and supplementary series the spectra of the generator $\tilde{K}_{0}$ are unbounded from below and above. One of the discrete series has a strictly positive spectrum of $\tilde{K}_{0}$ and the other a strictly negative one. In our case $\tilde{K}_{0}$ corresponds to the positive action variable $\tilde{I}$ and, therefore, ought to be a positive definite operator. This leaves only the positive discrete series of the irreducible 
unitary representations. These may be - formally - constructed as follows:

As the group $S O^{\uparrow}(1,2)$ is noncompact, its irreducible unitary representations are infinitedimensional. Different concrete representation Hilbert spaces will be discussed later in sec. 7.

In an irreducible unitary representation of the group the classical functions $\tilde{h}_{0}, \tilde{h}_{1}, \tilde{h}_{2}$ with their Lie algebra (10) correspond to self-adjoint operators $\tilde{K}_{0}, \tilde{K}_{1}, \tilde{K}_{2}$ which obey the commutation relations

$$
\left[\tilde{K}_{0}, \tilde{K}_{1}\right]=i \tilde{K}_{2}, \quad\left[\tilde{K}_{0}, \tilde{K}_{2}\right]=-i \tilde{K}_{1}, \quad\left[\tilde{K}_{1}, \tilde{K}_{2}\right]=-i \tilde{K}_{0}
$$

or, with the definitions

$$
\tilde{K}_{+}=\tilde{K}_{1}+i \tilde{K}_{2}, \quad \tilde{K}_{-}=\tilde{K}_{1}-i \tilde{K}_{2}
$$

we have

$$
\left[\tilde{K}_{0}, \tilde{K}_{+}\right]=\tilde{K}_{+}, \quad\left[\tilde{K}_{0}, \tilde{K}_{-}\right]=-\tilde{K}_{-}, \quad\left[\tilde{K}_{+}, \tilde{K}_{-}\right]=-2 \tilde{K}_{0} .
$$

The relations (235) are invariant under the replacement $\tilde{K}_{1} \rightarrow-\tilde{K}_{1}, \tilde{K}_{2} \rightarrow-\tilde{K}_{2}$ and $\tilde{K}_{1} \rightarrow$ $-\tilde{K}_{2}, \tilde{K}_{2} \rightarrow \tilde{K}_{1}$. The relations $(237)$ are invariant under $\tilde{K}_{+} \rightarrow \mu \tilde{K}_{+}, \tilde{K}_{-} \rightarrow \mu^{*} \tilde{K}_{-},|\mu|=1$, and under the transformations $\tilde{K}_{+} \leftrightarrow \tilde{K}_{-}, \tilde{K}_{0} \rightarrow-\tilde{K}_{0}$. In irreducible unitary representations with a scalar product $\left(f_{1}, f_{2}\right)$ the operator $\tilde{K}_{-}$is the adjoint operator of $\tilde{K}_{+}:\left(f_{1}, \tilde{K}_{+} f_{2}\right)=$ $\left(\tilde{K}_{-} f_{1}, f_{2}\right)$, and vice versa.

The (Casimir) operator

$$
\mathfrak{C}=\tilde{K}_{1}^{2}+\tilde{K}_{2}^{2}-\tilde{K}_{0}^{2}
$$

commutes with all three $\tilde{K}_{j}$ and therefore is a multiple of the identity operator in an irreducible representation. It obeys the relations

$$
\tilde{K}_{+} \tilde{K}_{-}=\mathfrak{C}+\tilde{K}_{0}\left(\tilde{K}_{0}-1\right), \quad \tilde{K}_{-} \tilde{K}_{+}=\mathfrak{C}+\tilde{K}_{0}\left(\tilde{K}_{0}+1\right) .
$$

Most unitary representations make use of the fact that $\tilde{K}_{0}$ is the generator of a compact group and that its eigenfunctions $g_{m}$ are normalizable elements of the associated Hilbert space $\mathcal{H}[69]$.

The relations (237) show that the $K_{ \pm}$act es creation and annihilation operators and they imply

$$
\begin{aligned}
\tilde{K}_{0} g_{m} & =m g_{m}, m \in \mathbb{R}, \quad\left(g_{m}, g_{m}\right)=1, \\
\tilde{K}_{0} \tilde{K}_{+} g_{m} & =(m+1) \tilde{K}_{+} g_{m}, \\
\tilde{K}_{0} \tilde{K}_{-} g_{m} & =(m-1) \tilde{K}_{-} g_{m},
\end{aligned}
$$

which, combined with (239), lead to

$$
\begin{aligned}
& \left(g_{m}, \tilde{K}_{+} \tilde{K}_{-} g_{m}\right)=\left(\tilde{K}_{-} g_{m}, \tilde{K}_{-} g_{m}\right)=\mathfrak{c}+m(m-1) \geq 0 \\
& \left(g_{m}, \tilde{K}_{-} \tilde{K}_{+} g_{m}\right)=\mathfrak{c}+m(m+1) \geq 0, \mathfrak{c}=\left(g_{m}, \mathfrak{C} g_{m}\right)
\end{aligned}
$$

It follows that

$$
\left(\tilde{K}_{+} g_{m}, \tilde{K}_{+} g_{m}\right)=2 m+\left(\tilde{K}_{-} g_{m}, \tilde{K}_{-} g_{m}\right) \geq 0 .
$$

As we assume that we have an irreducible representation the functions $g_{m}$ are eigenfunctions of the Casimir operator $\mathfrak{C}$ :

$$
\mathfrak{C} g_{m}=\mathfrak{c} g_{m} .
$$


The relations (240) - (245) show that the eigenvalues $m$ of $\tilde{K}_{0}$ in principle can be any real number, where, however, different eigenvalues differ by an integer.

As already said above: For the "principle" and the "complementary" series the spectrum of $\tilde{K}_{0}$ is unbounded from below and above [68], but as $\tilde{K}_{0}$ corresponds to the classical positive definite quantity $\tilde{I}$, these unitary representations are of no interest here.

Here the positive discrete series $D_{k}^{(+)}$of irreducible unitary representations are important. These are characterized by the property that there exists a lowest eigenvalue $m=k$ such that

$$
\tilde{K}_{0} g_{k}=k g_{k}, \quad \tilde{K}_{-} g_{k}=0 .
$$

Now the relations (243) and (245) imply

$$
\mathfrak{c}=k(1-k), \quad k>0, \quad m=k+n, n=0,1,2, \ldots
$$

That $k>0$ follows from Eq. (245) with $m=k, \tilde{K}_{-} g_{k}=0$, but $\left(\tilde{K}_{+} g_{k}, \tilde{K}_{+} g_{k}\right)>0$, because the scalar product is positive definite! Exploiting the relations (240) $-(242)$ yields

$$
\begin{aligned}
\tilde{K}_{0} g_{k, n} & =(k+n) g_{k, n}, k>0, n=0,1, \ldots, \quad\left(g_{k, n}, g_{k, n}\right)=1 \\
\tilde{K}_{+} g_{k, n} & =\mu_{n}[(2 k+n)(n+1)]^{1 / 2} g_{k, n+1}, \quad\left|\mu_{n}\right|=1 \\
\tilde{K}_{-} g_{k, n} & =\frac{1}{\mu_{n-1}}[(2 k+n-1) n]^{1 / 2} g_{k, n-1} .
\end{aligned}
$$

The phases $\mu_{n}$ guarantee that $\left(f_{1}, \tilde{K}_{+} f_{2}\right)=\left(\tilde{K}_{-} f_{1}, f_{2}\right)$. In most cases of interest $\mu_{n}$ is independent of $n$. Then one can absorb it into the definition of $K_{ \pm}$and forget about the phases $\mu_{n}$ !

Up to now $k$ may be any positive real number. A detailed analysis (see Appendix B) shows [68] that $k=1,2, \ldots$, for the group $S O^{\uparrow}(1,2)$ itself, $k=1 / 2,1,3 / 2, \ldots$, for the isomorphic groups $S p(2, \mathbb{R}) \cong S L(2, \mathbb{R}) \cong S U(1,1)$ and $k=1 / 4,1 / 2,3 / 4,1, \ldots$, for the metaplectic group $M p(2, \mathbb{R})$ we encountered above.

For the universal covering group $\tilde{G} \equiv S O_{[\infty]}^{\uparrow}(1,2)$ the "Bargmann index" $k$ may have any positive value $>0$. Further below we shall see that for an m-fold covering $S O_{[m]}^{\uparrow}(1,2)$ the index $k$ can take the rational values

$$
k=\frac{\mu}{m}, \quad \mu=1,2, \ldots .
$$

Here the natural number $m$ may be arbitrary large, i.e. the lowest value $k=1 / m$ can be made arbitrary small $>0$ !

As long as I do not specify the concrete Hilbert space used I shall employ Dirac's bracket notation and write $g_{k, n}=|k, n\rangle$. It follows from Eq. (250) that

$$
\begin{aligned}
|k, n\rangle= & \frac{1}{\sqrt{(2 k)_{n} n !}}\left(\tilde{K}_{+}\right)^{n}|k, 0\rangle \\
(2 k)_{n} \equiv & 2 k(2 k+1) \cdots(2 k+n-1)=\frac{\Gamma(2 k+n)}{\Gamma(2 k)}, \\
& (2 k)_{n=0}=1, \quad(1)_{n}=n !, \quad(-2 k)_{n}=(-1)^{n} n !\left(\begin{array}{c}
2 k \\
n
\end{array}\right) .
\end{aligned}
$$

The Casimir operator relation

$$
\tilde{K}_{1}^{2}+\tilde{K}_{2}^{2}=\tilde{K}_{0}^{2}+k(1-k) \mathbf{1}
$$


modifies the corresponding classical Pythagorean relation

$$
\tilde{h}_{1}^{2}+\tilde{h}_{2}^{2}=\tilde{h}_{0}^{2},
$$

unless $k=1$ ! So for a HO with $k=1 / 2$ "Pythagoras" is "violated" by quantum effects!

\subsection{The operators $\tilde{Q}$ and $\tilde{P}$ as functions of the operators $\tilde{K}_{j}$}

The relations (21), (27) , (215) and (216) as well show the dependence of the canonical coordinates $\tilde{q}$ and $\tilde{p}$ on the canonical coordinates $\varphi$ and $\tilde{I}$. It is important that a corresponding operator relation expresses the position operator $\tilde{Q}$ and the momentum operator $\tilde{P}$ in terms of the operators $\tilde{K}_{j}, j=0,1,2$. That this is indeed possible was already stated in the introduction. The relationship can be read off the Eqs. (249) - (251) as follows:

If we have annihilation and creation operators $a$ and $a^{\dagger}$ in a (Fock) Hilbert space with a number state basis $|n\rangle$ such that

$$
a|n\rangle=\sqrt{n}|n-1\rangle, \quad a^{\dagger}|n\rangle=\sqrt{n+1}|n+1\rangle, \quad\left[a, a^{\dagger}\right]=\mathbf{1},
$$

we can define

$$
\tilde{Q}=\frac{1}{\sqrt{2}}\left(a+a^{\dagger}\right), \quad \tilde{P}=\frac{i}{\sqrt{2}}\left(a^{\dagger}-a\right), \quad[\tilde{Q}, \tilde{P}]=i \mathbf{1} .
$$

The operators (258) have been used to construct non-linear realizations of the generators $\tilde{K}_{j}[27]$ :

$$
\tilde{K}_{0}=N+k \mathbf{1}, \quad \tilde{K}_{+}=a^{\dagger} \sqrt{N+2 k \mathbf{1}}, \quad \tilde{K}_{-}=\sqrt{N+2 k \mathbf{1}} a, \quad N=a^{\dagger} a .
$$

However, as I pointed out in Ref. [13], it is much more interesting to invert these relations:

\subsubsection{Operator version of the polar coordinates in the plane}

Now, as $k>0$ and the operator $\tilde{K}_{0}$ is positive definite in any irreducible unitary representation of the positive discrete series $D_{k}^{(+)}$, the operator

$$
B_{k}=\left(\tilde{K}_{0}+k\right)^{-1 / 2}
$$

is well-defined and self-adjoint. As

$$
B_{k}|k, n\rangle=(2 k+n)^{-1 / 2}|k, n\rangle,
$$

then according to the relations (249) - (251) (with $\left.\mu_{n}=1\right)$ the operators

$$
A_{(k)}(\overrightarrow{\tilde{K}})=B_{k} \tilde{K}_{-}, \quad A_{(k)}^{\dagger}(\overrightarrow{\tilde{K}})=\tilde{K}_{+} B_{k}
$$

have the properties

$$
\begin{aligned}
A_{(k)}|k, n\rangle=\sqrt{n}|k, n-1\rangle, & A_{(k)}^{\dagger}|k, n\rangle=\sqrt{n+1}|k, n+1\rangle, \\
A_{(k)}^{\dagger} A_{(k)}|k, n\rangle=n|k, n\rangle, & {\left[A_{(k)}, A_{(k)}^{\dagger}\right]=\mathbf{1} . }
\end{aligned}
$$

The actions of the operators (263) are independent of the (Bargmann) index $k$ which characterizes the irreducible representation of the group $S O^{\uparrow}(1,2)$ or one of its covering groups. So we may drop their index $(k)$. 
This $k$-independence is another manifestation of the Stone-von Neumann uniqueness theorem which says that - provided certain regularity conditions are fulfilled - all the irreducible representations of $\tilde{Q}$ and $\tilde{P}$ with the property (259) are unitarily equivalent, i.e. have the same matrix element whatever Hilbert space is employed!

Before drawing consequences let me derive the relation

$$
N=A^{\dagger}(\overrightarrow{\tilde{K}}) A(\overrightarrow{\tilde{K}})=\tilde{K}_{0}-k \mathbf{1}
$$

in a different way: If $f\left(\tilde{K}_{0}\right)$ is a "suitable" function of the operator $\tilde{K}_{0}$, then a repeated application of the relations (237) yields

$$
\tilde{K}_{-} f\left(\tilde{K}_{0}\right)=f\left(\tilde{K}_{0}+\mathbf{1}\right) \tilde{K}_{-}, \quad f\left(\tilde{K}_{0}\right) \tilde{K}_{+}=\tilde{K}_{+} f\left(\tilde{K}_{0}+\mathbf{1}\right)
$$

where "suitable" means that $f\left(\tilde{K}_{0}\right)$ and $f\left(\tilde{K}_{0}+\mathbf{1}\right)$ are both well-defined operators; We then have

$$
A^{\dagger} A=\tilde{K}_{+}\left(\tilde{K}_{0}+k\right)^{-1} \tilde{K}_{-}=\left(\tilde{K}_{0}+k-1\right)^{-1} \tilde{K}_{+} \tilde{K}_{-}=\left(\tilde{K}_{0}+k-1\right)^{-1}\left[k(1-k)+\tilde{K}_{0}\left(\tilde{K}_{0}-k\right)\right],
$$

where the first of the relations (239) has been used.

As $k(1-k)+\tilde{K}_{0}\left(\tilde{K}_{0}-k\right)=\left(\tilde{K}_{0}+k-1\right)\left(\tilde{K}_{0}-k\right)$ the Eq. (266) follows immediately.

Explicitly written in terms of the operators $\tilde{K}_{0}, \tilde{K}_{1}$ and $\tilde{K}_{2}$ we have

$$
\begin{aligned}
& \tilde{Q}(\overrightarrow{\tilde{K}})=\frac{1}{\sqrt{2}}\left(A+A^{\dagger}\right)=\frac{1}{\sqrt{2}}\left(\tilde{K}_{1} B_{k}+B_{k} \tilde{K}_{1}\right)+\frac{i}{\sqrt{2}}\left(\tilde{K}_{2} B_{k}-B_{k} \tilde{K}_{2}\right), \\
& \tilde{P}(\overrightarrow{\tilde{K}})=\frac{i}{\sqrt{2}}\left(A^{\dagger}-A\right)=\frac{i}{\sqrt{2}}\left(\tilde{K}_{1} B_{k}-B_{k} \tilde{K}_{1}\right)-\frac{1}{\sqrt{2}}\left(\tilde{K}_{2} B_{k}+B_{k} \tilde{K}_{2}\right) .
\end{aligned}
$$

These relations show that - contrary to the classical case (cf. Eqs. (215) and (216)) - the operators $\tilde{Q}$ and $\tilde{P}$ are not just proportional to $\tilde{K}_{1}$ and $\tilde{K}_{2}$, but contain mixtures of both!

\subsubsection{Two kinds of energy spectra for the quantum mechanical HO}

We now come to the crucial point of the whole paper:

Obviously the (dimensionless) $(\tilde{q}, \tilde{p})$-Hamiltonian

$$
\tilde{H}[\tilde{Q}(\overrightarrow{\tilde{K}}), \tilde{P}(\overrightarrow{\tilde{K}})]=\frac{1}{2} \tilde{Q}^{2}+\frac{1}{2} \tilde{P}^{2}=A^{\dagger} A+\frac{1}{2}
$$

obeys the eigenvalue equation

$$
\tilde{H}(\tilde{Q}, \tilde{P})|k, n\rangle=(n+1 / 2)|k, n\rangle .
$$

On the other hand we have for the $(\varphi, \tilde{I})$-Hamiltonian

$$
\tilde{H}(\vec{K})=\tilde{K}_{0}, \quad \vec{K}=\hbar\left(\tilde{K}_{0}, \tilde{K}_{1}, \tilde{K}_{2}\right), \quad H(\vec{K})=\hbar \omega \tilde{K}_{0}
$$

that

$$
\tilde{H}(\vec{K})|k, n\rangle=(n+k)|k, n\rangle, \quad k>0 .
$$

The last equation shows that the ground state energies of the Hamiltonian (273) in principle may take any real positive value! 
In sec. 3 we encountered the values $k=1$ (for $\left.S O^{\uparrow}(1,2)\right), k=1 / 2($ for $S p(2, \mathbb{R})$ ) and $k=1 / 4,3 / 4$ (for the 4 -fold covering group $M p(2, \mathbb{R})$ of $S O^{\uparrow}(1,2)$ ). One can show (see below and Appendix B) that for an $m$-fold covering $(m \in \mathbb{N})$ the lowest possible value for $k$ is $k=1 / m$. Thus, we can make $k>0$ as small as we like by going to higher and higher coverings.

These surprising new possibilities come, of course, from the non-trivial topological structure $\mathbb{R}^{2}-\{(0,0)\} \cong S^{1} \times \mathbb{R}^{+}$of the phase space $\mathcal{S}_{\varphi, \tilde{I}}$, a structure which is being "erased" when going over to the phase space $\mathcal{S}_{\tilde{q}, \tilde{p}}$ with its trivial topology $\mathbb{R}^{2}$ !

Actually, the more general eigenvalues of Eq. (274) are a consequence of the "richer" quantum theory of symplectic group $S p(2, \mathbb{R})$ of the plane which constitutes the "canonical" group of the phase space $\mathcal{S}_{\varphi, \tilde{I}}$.

It is, of course, of crucial importance, to look for this additional structure experimentally (see subsec. 1.3 of the Introduction)!!

If $k \neq 1 / 2$ then the two energy spectra

$$
E_{n}^{(q, p)}=\hbar \omega(n+1 / 2), \quad E_{k, n}^{(\varphi, I)}=\hbar \omega(n+k),
$$

are different and transitions between different levels should (in principle) be possible if the $E_{k, n}^{(\varphi, I)}$ - levels do appear at all in nature or can be produced in the laboratory! Of special interest here is the case where $0<k<1 / 2$ because then transitions from the $(q, p)$-ground state to a lower lying $(\varphi, I)$-level are in principle possible provided an appropriate dynamical mechanism is available. An obvious challenge is that for $k \neq 1 / 2$ the same states $|k, n\rangle$ belong to different energy eigenvalues of the operators $H(Q, P)$ and $H(\vec{K})$ ! Notice, however, that for $\tilde{H}(\vec{K})$ the "observables" $\tilde{K}_{0}, \tilde{K}_{1}$ and $\tilde{K}_{2}$ are the primary ones, whereas $\tilde{Q}$ and $\tilde{P}$ are "derived" or "composite" quantities, at least in the present context!

It may also happen, perhaps, that transitions between levels of the two different spectra are more or less strongly impeded and that, therefore, certain levels remain "in the dark"! (See also sec. 8).

\subsubsection{Time evolution and the ground states for different covering groups}

Let us look at the provoking situation from a slightly different point of view:

The unitary time evolution operator for the $(\varphi, \tilde{I})$-model of the HO is

$$
U(\tilde{t})=e^{-i \tilde{H} \tilde{t}}, \quad \tilde{H}=\tilde{K}_{0}, \quad \tilde{t}=\theta .
$$

This equation shows that the rotation angle $\theta$ can be identified with the time variable $\tilde{t}$ which - in principle - represents the universal covering space of the circle $S^{1}$.

From the commutation relations (235) and the formula (162) we get the (Heisenberg) eqs. of motion

$$
\begin{aligned}
U(-\tilde{t}) \tilde{K}_{1} U(\tilde{t}) & =\cos \tilde{t} \tilde{K}_{1}-\sin \tilde{t} \tilde{K}_{2}, \\
U(-\tilde{t}) \tilde{K}_{2} U(\tilde{t}) & =\sin \tilde{t} \tilde{K}_{1}+\cos \tilde{t} \tilde{K}_{2}, \\
U(-\tilde{t}) \tilde{K}_{+} U(\tilde{t}) & =e^{i \tilde{t}} \tilde{K}_{+}, \\
U(-\tilde{t}) \tilde{K}_{-} U(\tilde{t}) & =e^{-i \tilde{t}} \tilde{K}_{-} .
\end{aligned}
$$

As the operator (261) commutes with $U(\tilde{t})$ the creation and annihilation operators $A^{\dagger}$ and $A$ from Eq. (263) transform as $\tilde{K}_{+}$and $\tilde{K}_{-}$in Eqs. (279) and (280). This means that the 
position and momentum operators (269) and (270) have the usual time evolution:

$$
\begin{aligned}
U(-\tilde{t}) \tilde{Q} U(\tilde{t}) & =\cos \tilde{t} \tilde{Q}+\sin \tilde{t} \tilde{P} \\
U(-\tilde{t}) \tilde{P} U(\tilde{t}) & =-\sin \tilde{t} \tilde{Q}+\cos \tilde{t} \tilde{P}
\end{aligned}
$$

Here, all the explicit $k$-dependence has dropped out!

However, because of the relation (266) we have

$$
U(\tilde{t}=2 \pi)=e^{-2 \pi i k} \mathbf{1} .
$$

If $k$ is a positive rational number, $k=n / m, n, m \in \mathbb{N}$, then the unitary operator (283) belongs to the center of a unitary representation of a $m$-fold covering of $S O^{\uparrow}(1,2)$, the "lowest" representation of which is given by $k=1 / m$. Only for $k=1,2, \ldots$, the operator (283) is the identity operator, representing the identity of $S O^{\uparrow}(1,2)$. If $k=n / m$ then $U(\tilde{t}=m 2 \pi)$ is the corresponding identity operator.

Here we see, why the values of $k$ in the interval $(0,1]$ may be generically the most important ones in the context of the HO (see also the related discussions in Ref. [63]). The center

$$
\mathbb{Z}_{m}=\left\{e^{2 \pi i \mu / m}, \mu=1, \cdots, m\right\}
$$

of the m-fold covering may be generated by the single element

$$
e^{2 \pi i / m}
$$

For $\mu=m+1$ we obviously get the same element. Corresponding arguments apply to the unitary operator (283).

The relation (283) may also be interpreted in the following way: Applying the operator (276) to the ground state yields

$$
U(\tilde{t})|k, 0\rangle=e^{-i k \tilde{t}}|k, 0\rangle .
$$

As $\tilde{t}=\omega t$ can be used as an angle parametrizing one of the covering groups of the subgroup $S O(2)$, the interval

$$
T_{2 \pi, k}=\frac{2 \pi}{\omega_{k}}, \omega_{k} \equiv k \omega
$$

is the time the system needs in order to "run" through that group. So in a heuristic sense the index $k$ and the "angle" $\omega T_{2 \pi, k}$ are complementary! The larger the latter the smaller the former! I repeat: The index $k$ can principally be extremely small as long as it stays positive!

\subsubsection{The index $k$ in number states matrix elements}

The index $k$ plays a significant role in matrix elements of the operators $\tilde{K}_{j}, j=0,1$, 2 , with respect to the number states $|k, n\rangle$ :

It follows from

$$
\tilde{K}_{1}=\frac{1}{2}\left(\tilde{K}_{+}+\tilde{K}_{-}\right), \quad \tilde{K}_{2}=\frac{1}{2 i}\left(\tilde{K}_{+}-\tilde{K}_{-}\right),
$$

that

$$
\left\langle k, n\left|\tilde{K}_{j}\right| k, n\right\rangle=0, j=1,2
$$


and

$$
\left(\Delta \tilde{K}_{j}\right)_{k, n}^{2}=\left\langle k, n\left|\tilde{K}_{j}^{2}\right| k, n\right\rangle-\left\langle k, n\left|\tilde{K}_{j}\right| k, n\right\rangle^{2}=\frac{1}{2}\left(n^{2}+2 n k+k\right), j=1,2,
$$

so that

$$
\left(\Delta \tilde{K}_{1}\right)_{k, n}\left(\Delta \tilde{K}_{2}\right)_{k, n}=\frac{1}{2}\left(n^{2}+2 k n+k\right), \quad\left(\Delta \tilde{K}_{1}\right)_{k, n=0}\left(\Delta \tilde{K}_{2}\right)_{k, n=0}=\frac{k}{2},
$$

Thus, $\tilde{K}_{1}$ and $\tilde{K}_{2}$ have the same standard deviations ("uncertainties") and the product of these uncertainties in the ground state is given by $k / 2$, i.e. the smaller $k$ the smaller the minimal standard deviations!

For the operators $\tilde{Q}(\vec{K})$ and $\tilde{P}(\vec{K})$ we have

$$
\langle k, n|\tilde{Q}| k, n\rangle=0,\langle k, n|\tilde{P}| k, n\rangle=0,
$$

and

$$
(\Delta \tilde{Q})_{k, n}^{2}=\left\langle k, n\left|\tilde{Q}^{2}\right| k, n\right\rangle=n+1 / 2, \quad(\Delta \tilde{P})_{k, n}^{2}=\left\langle k, n\left|\tilde{P}^{2}\right| k, n\right\rangle=n+1 / 2,
$$

which are the usual $k$-independent relations, implying

$$
(\Delta \tilde{Q})_{k, n}(\Delta \tilde{P})_{k, n}=n+1 / 2 \text {. }
$$

\subsubsection{Space reflection and time reversal}

From Eqs. (277) and Eqs. (278), or Eqs. (281) and (282) we can infer the space reflection operator

$$
\Pi: \quad \Pi \tilde{Q} \Pi^{\dagger}=-\tilde{Q}, \quad \Pi \tilde{P} \Pi^{\dagger}=-\tilde{P}, \quad \Pi=U(\tilde{t}=-\pi)=e^{i \pi(N+k)} .
$$

Now

$$
\Pi^{2}=e^{2 \pi i k}, \quad \Pi|k, n\rangle=(-1)^{n} e^{i \pi k}|k, n\rangle,
$$

which shows the $k$-dependence of the phases associated with the so defined operator $\Pi$.

The antiunitary time reversal transformation $T$ (cf. Eq. (232) $)$ may be implemented by the substitutions

$$
T: \quad \tilde{K}_{0} \rightarrow \tilde{K}_{0}, \quad \tilde{K}_{1} \rightarrow \tilde{K}_{1}, \quad \tilde{K}_{2} \rightarrow-\tilde{K}_{2}, \quad i \rightarrow-i
$$

which imply

$$
T: \quad K_{ \pm} \rightarrow K_{ \pm}
$$

and leave the commutation relations (235) and (237) invariant. The transformations (297) imply the correct ones for the operators (269) and (270).

Contrary to what happens in the case of the canonical pair angle and orbital angular momentum where reflection and time reversal invariance are generally in conflict with fractional orbital angular momenta [25] this is not so for fractional ground state energies $\propto k$ of the HO!

Like in the case of the corresponding Poisson brackets (227) and (228) the commutators $\left[K_{ \pm}, A\right]$ etc. are rather complicated and will not be listed here. One can nevertheless define the following "squeezing" operator [70] "by hand":

$$
S=e^{-i V \gamma}, \quad V=\frac{i}{2}\left(A^{2}-\left(A^{\dagger}\right)^{2}\right), \quad \gamma \in \mathbb{R},
$$

which has the property

$$
S \tilde{Q} S^{\dagger}=e^{\gamma} \tilde{Q}, \quad S \tilde{P} S^{\dagger}=e^{-\gamma} \tilde{P}
$$




\subsection{Restoring the physical dimensions}

Up to now I have used dimensionless quantities, classical and quantum ones, as introduced in subsec. 2.1. Here I briefly summarize the main physical quantities with their dimensions restored. For the classical quantities the procedure is obvious from subsec. 2.1. So I confine myself to the operators and their eigenvalues:

The primary operators with the dimension of an action are

$$
K_{j}=\hbar \tilde{K}_{j}, \quad j=0,1,2, \quad K_{ \pm}=\hbar \tilde{K}_{ \pm} ;
$$

they have the commutation relations (cf. Eqs. (235) and (237))

$$
\left[K_{0}, K_{1}\right]=i \hbar K_{2}, \quad\left[K_{0}, K_{2}\right]=-i \hbar K_{1}, \quad\left[K_{1}, K_{2}\right]=-i \hbar K_{0},
$$

and

$$
\left[K_{0}, K_{+}\right]=\hbar K_{+}, \quad\left[K_{0}, K_{-}\right]=-\hbar K_{-}, \quad\left[K_{+}, K_{-}\right]=-2 \hbar K_{0} .
$$

We have, e.g.

$$
K_{0}|k, n\rangle=\hbar(n+k)|k, n\rangle .
$$

The Hamilton operator is given by

$$
H(\vec{K})=\omega K_{0}, \quad H|k, n\rangle=\hbar \omega(n+k)|k, n\rangle .
$$

The number operator remains dimensionless:

$$
N=\tilde{K}_{0}-k \mathbf{1} .
$$

The conventional annihilation and creation operators (263) should also remain dimensionless:

$$
A(\overrightarrow{\tilde{K}})=B_{k} \tilde{K}_{-}, \quad A^{\dagger}(\overrightarrow{\tilde{K}})=\tilde{K}_{+} B_{k}, \quad B_{k}=\left(\tilde{K}_{0}+k\right)^{-1 / 2}=(N+2 k)^{-1 / 2},
$$

so that

$$
\left[A, A^{\dagger}\right]=\mathbf{1} .
$$

The physical position and momentum operators are then given by (cf. Eqs. (38) and (39))

$$
Q=\frac{\lambda_{0}}{\sqrt{2}}\left(A^{\dagger}+A\right), \quad P=\frac{i \hbar}{\sqrt{2} \lambda_{0}}\left(A^{\dagger}-A\right), \quad[Q, P]=i \hbar, \quad \lambda_{0}=\sqrt{\frac{\hbar}{m \omega}} .
$$

\section{Three types of coherent states}

\subsection{Definition and physical interpretation}

It is well-known [71] that one can associate three different types of coherent states (CS) with the Lie algebra of the $\tilde{K}_{j}, j=0,1,2$, in a representation $D_{k}^{(+)}$: Barut-Girardello, Perelomov and the conventional Schrödinger-Glauber coherent states. The three kinds of CS may be defined by the relations

$$
\begin{aligned}
\tilde{K}_{-}|k, z\rangle & =z|k, z\rangle, \quad z=|z| e^{-i \phi} \in \mathbb{C} \\
E_{k,-}|k, \lambda\rangle & =\lambda|k, \lambda\rangle, \quad \lambda=|\lambda| e^{-i \theta} \in \mathbb{D}, \\
E_{k,-} & =\left(\tilde{K}_{0}+k\right)^{-1} \tilde{K}_{-}, \quad \mathbb{D}=\{\lambda \in \mathbb{C}, \quad|\lambda|<1\} \\
A|k, \alpha\rangle & =\alpha|k, \alpha\rangle, \quad A=B_{k} \tilde{K}_{-}, \quad \alpha=|\alpha| e^{-i \beta} \in \mathbb{C} .
\end{aligned}
$$


The minus-sign for the phases of the complex numbers is mere convenience 2 .

Expanding with respect to a number basis $|k, n\rangle$ yields [71]

$$
\begin{aligned}
|k, z\rangle= & \frac{1}{\sqrt{g_{k}\left(|z|^{2}\right)}} \sum_{n=0}^{\infty} \frac{z^{n}}{\sqrt{(2 k)_{n} n !}}|k, n\rangle, \\
& g_{k}\left(|z|^{2}\right)=\sum_{n=0}^{\infty} \frac{|z|^{2 n}}{(2 k)_{n} n !}=\Gamma(2 k)|z|^{1-2 k} I_{2 k-1}(2|z|) ; \\
|k, \lambda\rangle= & \left(1-|\lambda|^{2}\right)^{k} \sum_{n=0}^{\infty}\left(\frac{(2 k)_{n}}{n !}\right)^{1 / 2} \lambda^{n}|k, n\rangle, \quad|\lambda|<1 ; \\
|k, \alpha\rangle= & e^{-|\alpha|^{2} / 2} \sum_{n=0}^{\infty} \frac{\alpha^{n}}{\sqrt{n !}}|k, n\rangle .
\end{aligned}
$$

The function $I_{\nu}(x)$ in Eq. (315) is the usual modified Bessel function of the first kind:

$$
I_{\nu}(x)=\left(\frac{x}{2}\right)^{\nu} \sum_{n=0}^{\infty} \frac{1}{n ! \Gamma(\nu+n+1)}\left(\frac{x}{2}\right)^{2 n} .
$$

The series (314) - (317) are formal ones the convergence properties of which can be specified once the number states and their Hilbert space are given explicitly.

The physical interpretation of the complex numbers $z, \lambda$ and $\alpha$ can be deduced from the following expectation values:

\subsubsection{Barut-Girardello coherent states}

$$
\begin{aligned}
\left\langle\tilde{K}_{0}\right\rangle_{k, z} \equiv & \left\langle k, z\left|\tilde{K}_{0}\right| k, z\right\rangle=k+|z| \rho_{k}(|z|), \\
& \rho_{k}(|z|)=\frac{I_{2 k}(2|z|)}{I_{2 k-1}(2|z|)}<1, \quad k \geq 1 / 4, \\
\left(\Delta \tilde{K}_{0}\right)_{k, z}^{2}= & |z|^{2}\left[1-\rho_{k}^{2}(|z|)\right]+(1-2 k)|z| \rho_{k}(|z|), \\
\langle N\rangle_{k, z} \equiv & \bar{n}_{k, z}=|z| \rho_{k}(|z|), \quad N=\tilde{K}_{0}-k \mathbf{1}, \\
\left\langle N^{2}\right\rangle_{k, z}= & |z|^{2}+(1-2 k)|z| \rho_{k}(|z|), \\
\left\langle\tilde{K}_{1}\right\rangle_{k, z}= & \frac{1}{2}\left(z^{*}+z\right)=\Re(z)=|z| \cos \phi \\
\left\langle\tilde{K}_{2}\right\rangle_{k, z}= & \frac{1}{2 i}\left(z^{*}-z\right)=-\Im(z)=|z| \sin \phi, \\
\left(\Delta \tilde{K}_{1}\right)_{k, z}^{2}=\left(\Delta \tilde{K}_{2}\right)_{k, z}^{2}= & \frac{1}{2}\left\langle\tilde{K}_{0}\right\rangle_{k, z}, \\
\tan \phi= & \left\langle\tilde{K}_{2}\right\rangle_{k, z} /\left\langle\tilde{K}_{1}\right\rangle_{k, z}
\end{aligned}
$$

The behaviour of the ratio $\rho_{k}$ from Eq. (320) for all $k>0$ is discussed in Appendix C.

\footnotetext{
${ }^{2}$ Ref. [13] has the opposite sign convention.
} 


\subsubsection{Perelomov coherent states}

$$
\begin{aligned}
&\left\langle\tilde{K}_{0}\right\rangle_{k, \lambda} \equiv\left\langle k, \lambda\left|\tilde{K}_{0}\right| k, \lambda\right\rangle=k \frac{1+|\lambda|^{2}}{1-|\lambda|^{2}}=k \cosh |w| \\
& w=|w| e^{-i \theta} \in \mathbb{C}, \lambda=\tanh (|w| / 2) e^{-i \theta},|w|=\ln \left(\frac{1+|\lambda|}{1-|\lambda|}\right), \\
&\langle N\rangle_{k, \lambda} \equiv \bar{n}_{k, \lambda}=k(\cosh |w|-1), \\
& \Rightarrow|\lambda|^{2}=\frac{\bar{n}_{k, \lambda}}{\bar{n}_{k, \lambda}+2 k}, k \sinh |w|=\sqrt{\bar{n}_{k, \lambda}\left(\bar{n}_{k, \lambda}+2 k\right)} \\
&\left(\Delta \tilde{K}_{0}\right)_{k, \lambda}^{2}=\frac{k}{2} \sinh ^{2}|w|=\frac{1}{2 k} \bar{n}_{k, \lambda}\left(\bar{n}_{k, \lambda}+2 k\right) \\
&\left\langle\tilde{K}_{1}\right\rangle_{k, \lambda}=2 k \frac{|\lambda|}{1-|\lambda|^{2}} \cos \theta=k \sinh |w| \cos \theta \\
&\left(\Delta \tilde{K}_{1}\right)_{k, \lambda}^{2}=\frac{k}{2} \frac{1+2 \cos 2 \theta|\lambda|^{2}+|\lambda|^{4}}{\left(1-|\lambda|^{2}\right)^{2}} \\
&\left\langle\tilde{K}_{2}\right\rangle_{k, \lambda}=2 k \frac{|\lambda|}{1-|\lambda|^{2}} \sin \theta=k \sinh |w| \sin \theta \\
&\left(\Delta \tilde{K}_{2}\right)_{k, \lambda}^{2}=\frac{k}{2} \frac{1-2 \cos 2 \theta|\lambda|^{2}+|\lambda|^{4}}{\left(1-|\lambda|^{2}\right)^{2}} \\
&\left\langle\tilde{K}_{0}\right\rangle_{k, \lambda}^{2}=\left\langle\tilde{K}_{1}\right\rangle_{k, \lambda}^{2}+\left\langle\tilde{K}_{2}\right\rangle_{k, \lambda}^{2}+k^{2} \\
& \tan \theta=\left\langle\tilde{K}_{2}\right\rangle_{k, \lambda} /\left\langle\tilde{K}_{1}\right\rangle_{k, \lambda}
\end{aligned}
$$

\subsubsection{Schrödinger-Glauber coherent states}

$$
\begin{aligned}
\langle\tilde{Q}\rangle_{k, \alpha}= & \sqrt{2} \Re(\alpha)=\tilde{q}=\sqrt{2}|\alpha| \cos \beta, \\
\langle\tilde{P}\rangle_{k, \alpha}= & \sqrt{2} \Im(\alpha)=\tilde{p}=-\sqrt{2}|\alpha| \sin \beta, \\
\langle\tilde{H}(\tilde{Q}, \tilde{P})\rangle_{k, \alpha}= & |\alpha|^{2}+1 / 2, \\
\left\langle\tilde{K}_{0}\right\rangle_{k, \alpha}= & \langle N\rangle_{k, \alpha}+k=|\alpha|^{2}+k, \quad N=\tilde{K}_{0}-k \mathbf{1} \\
\left\langle\tilde{K}_{1}\right\rangle_{k, \alpha}= & |\alpha| \cos \beta\langle k, \alpha|\sqrt{N+2 k}| k, \alpha\rangle, \\
\left\langle\tilde{K}_{2}\right\rangle_{k, \alpha}= & |\alpha| \sin \beta\langle k, \alpha|\sqrt{N+2 k}| k, \alpha\rangle, \\
& \langle k, \alpha|\sqrt{N+2 k}| k, \alpha\rangle=e^{-|\alpha|^{2}} \sum_{n=0}^{\infty} \sqrt{2 k+n} \frac{|\alpha|^{2 n}}{n !} \equiv h_{1}^{k}(|\alpha|), \\
\tan \beta= & \left\langle\tilde{K}_{2}\right\rangle_{k, \alpha} /\left\langle\tilde{K}_{1}\right\rangle_{k, \alpha} .
\end{aligned}
$$

\subsubsection{Physical interpretation of the complex variables}

\section{Barut-Girardello states}

Eqs. (324) and (325) show that we can interpret $\Re(z)$ as the classical variable $\tilde{h}_{1}$ and $\Im(z)$ as $\tilde{h}_{2}$, i.e. we have

$$
z=\tilde{h}_{1}+i \tilde{h}_{2}=\tilde{h}_{+}=|z| e^{-i \phi},|z|=\tilde{I}>0, \phi=\varphi
$$


Deviations from the classical value $|z|$ etc. in the relations (319) and (321) - (323) are controlled by the ratio $\rho_{k}$. It has the limiting values [71]

$$
\rho_{k}(|z|) \rightarrow \frac{|z|}{2 k}\left(1-\frac{|z|^{2}}{2 k(2 k+1)}\right) \text { for }|z| \rightarrow 0,
$$

and for very large $|z|$, the correspondence limit, we get

$$
\begin{aligned}
\rho_{k}(|z|) & \asymp 1-\frac{4 k-1}{4|z|}+\frac{16\left(k^{2}-k\right)+3}{32|z|^{2}}+O\left(|z|^{-3}\right), \\
\rho_{k}^{2}(|z|) & \asymp 1-\frac{4 k-1}{2|z|}+\frac{8 k^{2}-6 k+1}{4|z|^{2}}+O\left(|z|^{-3}\right) \text { for }|z| \rightarrow \infty .
\end{aligned}
$$

The last two relations imply that for large $|z|$

$$
\begin{aligned}
\left\langle K_{0}\right\rangle_{k, z} & \asymp|z|+\frac{1}{4}+O\left(|z|^{-1}\right), \\
\left(\Delta K_{0}\right)_{k, z}^{2} & \asymp \frac{1}{2}|z|+O\left(|z|^{-1}\right), \\
\bar{n}_{k, z} & \asymp|z|+\frac{1}{4}-k+O\left(|z|^{-1}\right), \\
(\Delta N)_{k, z}^{2} & \asymp \frac{1}{2}|z|+O\left(|z|^{-1}\right) \asymp \frac{1}{2} \bar{n}_{k, z},
\end{aligned}
$$

\section{Perelomov states}

Here the situation is different from the previous one: The expectation values (333) and (335) are proportional to the index $k$, a completely non-classical quantity. This suggests to divide out the factor $k$ and make the "classical" interpretations

$$
\tilde{h}_{1}=\tilde{I} \cos \theta, \quad \tilde{h}_{2}=-\tilde{I} \sin \theta, \quad \tilde{I}=\sinh |w|, \quad|w|>0, \quad \theta=\varphi .
$$

It means that

$$
|w|=\ln \left(\tilde{I}+\sqrt{\tilde{I}^{2}+1}\right), \quad|\lambda|=\tanh (|w| / 2)=\frac{\tilde{I}}{1+\sqrt{\tilde{I}^{2}+1}},
$$

so that

$$
\lambda=\frac{\tilde{h}_{1}+i \tilde{h}_{2}}{1+\sqrt{\tilde{I}^{2}+1}} .
$$

It follows that the expectation value (328) of $\tilde{K}_{0}$ approaches the value $k \tilde{I}$ in the classical limit for which $|w| \rightarrow \infty$ or $|\lambda| \rightarrow 1^{-}$.

It is remarkable that the above expectation values with respect to the states $|k, \lambda\rangle$ are all proportional to $k$, i.e. they have a sensitive $k$-dependence. This may be of interest for experimental tests.

\section{Schrödinger-Glauber states}

The first three of the expectation values (339) - (344) are well-known. They show that

$$
|\alpha|^{2}=\tilde{I}, \beta=\varphi \text {. }
$$

The others have been discussed in subsec. 3.3 of Ref. [13]. 


\section{Measuring the phases}

The three relations (327), (338) and (346) show that the operators $\tilde{K}_{1}$ and $\tilde{K}_{2}$ can be used in order to "measure" phases of complex amplitudes.

\subsection{Generation from the ground state}

The coherent states (314) - (317) may be generated from the ground state $|k, 0\rangle$ by unitary or similar operators. The unitary operators are also useful for the experimental generation of those states (see subsec. 6.5). Another problem is the appropriate experimental preparation of the ground state $|k, 0\rangle$ on which the unitary operators act.

\subsubsection{Schrödinger-Glauber states}

The coherent states (317) can be generated from the groundstate $|k, 0\rangle$ by the unitary operator

$$
U_{S G}=e^{\alpha A^{\dagger}-\alpha^{*} A}=e^{-|\alpha|^{2} / 2} e^{\alpha A^{\dagger}} e^{-\alpha^{*} A}, \quad U_{S G}|k, 0\rangle=|k, \alpha\rangle,
$$

which is well-known for the case $k=1 / 2$. The operator (359) has the "displacement" (translation) properties

$$
U_{S G}^{\dagger} A U_{S G}=A+\alpha, \quad U_{S G}^{\dagger} A^{\dagger} U_{S G}=A^{\dagger}+\alpha^{*},
$$

so that

$$
U_{S G}^{\dagger} A^{\dagger} A U_{S G}=A^{\dagger} A+\alpha A^{\dagger}+\alpha^{*} A+|\alpha|^{2},
$$

with

$$
\left\langle k, 0\left|U_{S G}^{\dagger} A^{\dagger} A U_{S G}\right| k, 0\right\rangle=|\alpha|^{2} .
$$

If $\alpha$ becomes time-dependent, the transformed number operator (361) corresponds to a driven harmonic oscillator, i.e. an oscillator coupled to an external source [72]. Such external sources are actually used in order to generate these coherent states experimentally [73]. In textbooks and articles laser light is frequently mentioned as being in a coherent state. The characteristic Poisson distribution of the associated photons is, however, only reached for lasers well above threshhold [74].

\subsubsection{Perelomov states}

The states (316) can be generated from $|k, 0\rangle$ by the unitary operator [75]

$$
U_{P}=e^{(w / 2) \tilde{K}_{+}-\left(w^{*} / 2\right) \tilde{K}_{-}}=e^{\lambda \tilde{K}_{+}} e^{\ln \left(1-|\lambda|^{2}\right) \tilde{K}_{0}} e^{-\lambda^{*} \tilde{K}_{-}}, \quad U_{P}|k, 0\rangle=|k, \lambda\rangle,
$$

where the complex number $w$ is the same as in Eq. (329). 
Instead of the displacements (360) we here have the Lorentz transformations [76]

$$
\begin{aligned}
U_{P}^{\dagger} \tilde{K}_{0} U_{P}= & \cosh |w| \tilde{K}_{0}+ \\
& +\frac{1}{2} \sinh |w|\left(e^{-i \theta} \tilde{K}_{+}+e^{i \theta} \tilde{K}_{-}\right), \\
U_{P}^{\dagger} \tilde{K}_{+} U_{P}= & \frac{1}{2}(\cosh |w|+1) \tilde{K}_{+}+ \\
& +\frac{1}{2} e^{2 i \theta}(\cosh |w|-1) \tilde{K}_{-}+e^{i \theta} \sinh |w| \tilde{K}_{0}, \\
U_{P}^{\dagger} \tilde{K}_{-} U_{P}= & \frac{1}{2}(\cosh |w|+1) \tilde{K}_{-}+ \\
& +\frac{1}{2} e^{-2 i \theta}(\cosh |w|-1) \tilde{K}_{+}+e^{-i \theta} \sinh |w| \tilde{K}_{0},
\end{aligned}
$$

The relation corresponding to Eq. (362) here is

$$
\left\langle k, 0\left|U_{P}^{\dagger} \tilde{K}_{0} U_{P}\right| k, 0\right\rangle=k \cosh |w| .
$$

In terms of the vectors

$$
\vec{K}_{\perp}=\left(\tilde{K}_{1}, \tilde{K}_{2}\right), \quad \vec{n}=(\cos \theta, \sin \theta),
$$

these relations may be written as

$$
\begin{aligned}
U_{P}^{\dagger} \tilde{K}_{0} U_{P} & =\cosh |w| \tilde{K}_{0}+\sinh |w|\left(\vec{n} \cdot \vec{K}_{\perp}\right), \\
U_{P}^{\dagger} \vec{K}_{\perp} U_{P} & =\vec{K}_{\perp}+(\cosh |w|-1)\left(\vec{n} \cdot \vec{K}_{\perp}\right) \vec{n}+\sinh |w| \vec{n} \tilde{K}_{0} .
\end{aligned}
$$

The operator (363) now generates interaction terms for the original $\tilde{K}_{0}$ which are proportional to $\tilde{K}_{+}$and $\tilde{K}_{-}$, or to $\tilde{K}_{1}$ and (or) $\tilde{K}_{2}$. (Their classical counterparts for $\theta=0$ and $\theta=\pi / 2$ were briefly discussed in subsec. 2.3.) The use of the induced interaction term in Eq. (369) in theoretical descriptions of experiments will be discussed in subsec. 6.5.

\subsubsection{Barut-Girardello states}

Here the situation appears to be more complicated, because no corresponding unitary operator has been derived by now. The present situation is as follows [77]:

Because of the relation (284) we can write

$$
\sum_{n=0}^{\infty} \frac{z^{n}}{\sqrt{(2 k)_{n} n !}}|k, n\rangle=\sum_{n=0}^{\infty} \frac{z^{n}}{(2 k)_{n} n !}\left(\tilde{K}_{+}\right)^{n}|k, 0\rangle .
$$

As

$$
\frac{1}{(2 k)_{n}}\left(\tilde{K}_{+}\right)^{n}|k, 0\rangle=\left(E_{k,+}\right)^{n}|k, 0\rangle, \quad E_{k,+}=\tilde{K}_{+}\left(\tilde{K}_{0}+k\right)^{-1}=\left(E_{k,-}\right)^{\dagger},
$$

where $E_{k,-}$ as in Eq. (312), we have

$$
\sum_{n=0}^{\infty} \frac{z^{n}}{\sqrt{(2 k)_{n} n !}}|k, n\rangle=F_{k}(z)|k, 0\rangle, \quad F_{k}(z)=e^{z E_{k,+}} .
$$


The non-unitary operators $F_{k}(z)$ and $F_{k}^{\dagger}(z)=\exp \left(z^{*} E_{k,-}\right)$ have the following properties:

$$
\left\langle k, 0\left|F_{k}^{\dagger}(z) F_{k}(z)\right| k, 0\right\rangle=g_{k}\left(|z|^{2}\right)>0, \quad F_{k}^{\dagger}(z)|k, 0\rangle=|k, 0\rangle, \quad F_{k}^{\dagger}(z)|k, \lambda\rangle=e^{z^{*} \lambda}|k, \lambda\rangle,
$$

where $g_{k}\left(|z|^{2}\right)$ is defined in Eq. (315) and $|k, \lambda\rangle$ in Eq. (311).

Thus, we have

$$
F_{k}(z)|k, 0\rangle=\sqrt{g_{k}\left(|z|^{2}\right)}|k, z\rangle,
$$

i.e. $F_{k}(z)$ generates the unnormalized Barut-Girardello states. It corresponds to the similar generating parts

$$
e^{\alpha A^{\dagger}}, \quad e^{\lambda \tilde{K}_{+}}
$$

of the unitary operators (359) and (363) for the unnormalized Schrödinger-Glauber and Perelomov states. But, contrary to $A^{\dagger}$ and $\tilde{K}_{+}$the operators $E_{k,+}$ and $E_{k,-}$ are not elements of a Lie algebra. They have - among others more complicated ones - the commutators

$$
\left[E_{k,-}, E_{k,+}\right]=\frac{2 k-1}{\left(\tilde{K}_{0}+k\right)\left(\tilde{K}_{0}+k-1\right)}, \quad\left[\tilde{K}_{-}, E_{k,+}\right]=1, \quad\left[E_{k,-}, \tilde{K}_{+}\right]=1
$$

It follows from the completeness relation (396) and the last of the relations (374) that one has for $F_{k}^{\dagger}(z) F_{k}(z)$ the "spectral representation"

$$
F_{k}^{\dagger}(z) F_{k}(z)=\int_{\mathbb{D}} d \mu_{k}(\lambda) e^{z^{*} \lambda+z \lambda^{*}}|k, \lambda\rangle\langle k, \lambda|
$$

\subsubsection{Transitions between Perelomov and Barut-Girardello coherent states}

Notice that, according to Eqs. (314) and (316),

$$
\begin{aligned}
\langle k, \lambda \mid k, z\rangle & =\frac{\left(1-|\lambda|^{2}\right)^{k}}{\sqrt{g_{k}\left(|z|^{2}\right)}} e^{\lambda^{*} z} \\
p_{k}(\lambda \leftrightarrow z) & =|\langle k, \lambda \mid k, z\rangle|^{2}=\frac{\left(1-|\lambda|^{2}\right)^{2 k}}{g_{k}\left(|z|^{2}\right)} e^{2|\lambda||z| \cos (\phi-\theta)} .
\end{aligned}
$$

As $[71]$

$$
g\left(|z|^{2}\right) \asymp \frac{\Gamma(2 k)}{2 \sqrt{\pi}}|z|^{1 / 2-2 k} e^{2|z|}[1+O(1 /|z|)] \text { for large }|z|,
$$

we get for the transition probability in the (classical) limit of large $|z|$ :

$$
p_{k}(\lambda \leftrightarrow z) \asymp \frac{2 \sqrt{\pi}}{\Gamma(2 k) \sqrt{|z|}}\left[|z|\left(1-|\lambda|^{2}\right)\right]^{2 k} e^{-2|z|[1-|\lambda| \cos (\phi-\theta)]} \text { for large }|z| .
$$

According to Eqs. (347) and (347) we have

$$
|\lambda| \asymp 1-\frac{k}{|z|} \text { for large }|z| .
$$

Inserting this approximation for $|\lambda|$ into the relation (381) yields in leading order for large $|z|$

$$
p_{k}(\lambda \leftrightarrow z) \asymp \frac{2 \sqrt{\pi}(2 k)^{2 k}}{\Gamma(2 k) \sqrt{|z|}} e^{-2|z|[1-\cos (\phi-\theta)]} \text { for }|z| \rightarrow \infty .
$$


Expanding $\cos (\phi-\theta)$ around $(\phi-\theta)=0$ gives an approximate Gaussian distribution for $p_{k}(\lambda \leftrightarrow z)$ :

$$
p_{k}(\lambda \leftrightarrow z) \asymp \frac{2 \sqrt{\pi}(2 k)^{2 k}}{\Gamma(2 k) \sqrt{|z|}} e^{-|z|(\phi-\theta)^{2}} \text { for }|z| \rightarrow \infty .
$$

This shows that for a given large $|z|$ the transition probability is maximal for $\phi=\theta$.

On the other hand, it follows from

$$
\lim _{k \rightarrow 0^{+}}(2 k)^{2 k}=1, \quad \Gamma(2 k) \rightarrow \frac{1}{2 k} \text { for } k \rightarrow 0^{+},
$$

that $p_{k}$ becomes very small for very small $k$.

Properties of the matrix elements $\langle k, \alpha \mid k, z\rangle$ and $\langle k, \alpha \mid k, \lambda\rangle$ are discussed in chap. 3 of Ref. [13]. In the special case $k=1 / 2$ they are described in subsec. 7.1 below.

\subsection{Time evolution}

It follows from

$$
U(\tilde{t})|k, n\rangle=e^{-i(n+k) \tilde{t}}|k, n\rangle, \quad U(\tilde{t})=e^{-i \tilde{K}_{0} \tilde{t}}
$$

that

$$
\begin{array}{rll}
U(\tilde{t})|k, z\rangle & =e^{-i k \tilde{t}}|k, z(\tilde{t})\rangle, & z(\tilde{t})=z e^{-i \tilde{t}} \\
U(\tilde{t})|k, \lambda\rangle & =e^{-i k \tilde{t}}|k, \lambda(\tilde{t})\rangle, & \lambda(\tilde{t})=\lambda e^{-i \tilde{t}} \\
U(\tilde{t})|k, \alpha\rangle & =e^{-i k \tilde{t}}|k, \alpha(\tilde{t})\rangle, & \alpha(\tilde{t})=\alpha e^{-i \tilde{t}}
\end{array}
$$

These equations show that the time evolution does not change the form of the coherent states. It essentially shifts only the phases of the complex numbers $z, \lambda$ and $\alpha$ linearly in time:

$$
\phi \rightarrow \phi+\tilde{t}, \quad \theta \rightarrow \theta+\tilde{t}, \quad \beta \rightarrow \beta+\tilde{t} .
$$

\subsection{Some general properties}

I finally list some general properties of the above coherent states which are very useful for applications:

\subsubsection{Scalar products}

Using the orthonormality of the number states $|k, n\rangle$ two different states within one of the types listed in Eqs. (314) - (317) have the scalar product

$$
\begin{aligned}
\left\langle k, z_{2} \mid k, z_{1}\right\rangle & =\sum_{n=0}^{\infty}\left\langle k, z_{2} \mid k, n\right\rangle\left\langle k, n \mid k, z_{1}\right\rangle=\frac{g_{k}\left(z_{2}^{*} z_{1}\right)}{\sqrt{g_{k}\left(\left|z_{2}\right|^{2}\right) g_{k}\left(\left|z_{1}\right|^{2}\right)}} \\
\left\langle k, \lambda_{2} \mid k, \lambda_{1}\right\rangle & =\left(1-\left|\lambda_{1}\right|^{2}\right)^{k}\left(1-\left|\lambda_{2}\right|^{2}\right)^{k}\left(1-\lambda_{2}^{*} \lambda_{1}\right)^{-2 k} \\
\left\langle\alpha_{2} \mid \alpha_{1}\right\rangle & =e^{-\left(\left|\alpha_{2}\right|^{2}+\left|\alpha_{1}\right|^{2}\right) / 2} e^{\alpha_{2}^{*} \alpha_{1}}
\end{aligned}
$$

Different states are not orthogonal, but they are "complete" in the sense that they provide a resolution of the identity as follows [71]: 


\subsubsection{Completeness}

$$
\begin{aligned}
\int_{\mathbb{C}} d \mu_{k}(z)|k, z\rangle\langle k, z| & =\mathbf{1} \\
d \mu_{k}(z) & =\frac{2}{\pi \Gamma(2 k)}|z|^{2 k} K_{2 k-1}(2|z|) g_{k}\left(|z|^{2}\right) d|z| d \phi, k>0 ; \\
\int_{\mathbb{D}} d \mu_{k}(\lambda)|k, \lambda\rangle\langle k, \lambda| & =\mathbf{1} \\
d \mu_{k}(\lambda) & =\frac{2 k-1}{\pi}\left(1-|\lambda|^{2}\right)^{-2}|\lambda| d|\lambda| d \theta, k>1 / 2 ; \\
\frac{1}{\pi} \int_{\mathbb{C}} d^{2} \alpha|\alpha\rangle\langle\alpha| & =\mathbf{1}, \\
d^{2} \alpha & =d \Re(\alpha) d \Im(\alpha) .
\end{aligned}
$$

The modified Bessel function of the third kind $K_{\nu}(2|z|)$ (cf. Ref. [79]) in the measure (395) has the property $K_{-\nu}(2|z|)=K_{\nu}(2|z|)$ which makes the measure well-defined for $k>0$, because in the limit $|z| \rightarrow 0$ one has

$$
\tilde{K}_{0}(2|z|) \rightarrow \ln (1 /|z|), K_{\nu}(2|z|) \rightarrow \frac{\Gamma(\nu)|z|^{-\nu}}{2}+\frac{\Gamma(-\nu)|z|^{\nu}}{2} \text { for } 0<|\nu|<1
$$

and

$$
\tilde{K}_{1}(2|z|) \rightarrow 1 /(2|z|)+|z| \ln |z|, K_{\nu}(2|z|) \rightarrow \Gamma(|\nu|)|z|^{-|\nu|} \text { for }|\nu|>1 .
$$

The extension of Hilbert spaces with the measure (397) for states $|k, \lambda\rangle$ with $0<k \leq 1 / 2$ will be discussed below.

The relation (398) holds for all $k>0$.

\subsubsection{Hilbert spaces of holomorphic functions associated with the three types of coherent states}

It is well-known that the three types of coherent states (314) - (317) can be associated with Hilbert spaces of holomorphic functions [80], the (normalized!) basis elements of which are given by the coefficients under the sums of the expensions with respect to the states $|k, n\rangle[71]$ :

\section{Barut-Girardello holomorphic functions}




$$
\begin{aligned}
\left(f_{2}, f_{1}\right)_{k, z} & \equiv \int_{\mathbb{C}} d \hat{\mu}_{k}(z) f_{2}^{*}(z) f_{1}(z), \\
d \hat{\mu}_{k}(z) & =\frac{2}{\pi \Gamma(2 k)}|z|^{2 k} K_{2 k-1}(2|z|) d|z| d \phi, k>0, \\
\hat{f}_{k, n}(z) & =\frac{z^{n}}{\sqrt{(2 k)_{n} n !}, \quad\left(\hat{f}_{k, n_{2}}, \hat{f}_{k, n_{1}}\right)_{k, z}=\delta_{n_{2} n_{1}},} \\
\Delta_{k}\left(z_{2}^{*}, z_{1}\right)=\sum_{n=0}^{\infty} \hat{f}_{k, n}^{*}\left(z_{2}\right) \hat{f}_{k, n}\left(z_{1}\right) & =g_{k}\left(z_{2}^{*} z_{1}\right), \\
\int_{\mathbb{C}} d \hat{\mu}_{k}\left(z_{2}\right) \Delta_{k}\left(z_{2}^{*}, z_{1}\right) \hat{f}_{k, n}\left(z_{2}\right) & =\hat{f}_{k, n}\left(z_{1}\right), \\
\int_{\mathbb{C}} d \hat{\mu}_{k}\left(z_{2}\right) \Delta_{k}\left(z_{2}^{*}, z_{1}\right) f\left(z_{2}\right) & =f\left(z_{1}\right), f(z)=\sum_{n=0}^{\infty} a_{n} z^{n}, \\
\int_{\mathbb{C}} d \hat{\mu}_{k}(z) \Delta_{k}\left(z_{2}^{*}, z\right) \Delta_{k}\left(z^{*}, z_{1}\right) & =\Delta_{k}\left(z_{2}^{*}, z_{1}\right), \\
\left(f_{2}, f_{1}\right)_{k, z}=\sum_{n=0}^{\infty}(2 k)_{n} n ! a_{n, 2}^{*} a_{n, 1}, & f_{j}(z)=\sum_{n=0}^{\infty} a_{n, j} z^{n}, j=1,2 .
\end{aligned}
$$

Because of the properties (405) - (407) the function $\Delta_{k}\left(z_{2}^{*}, z_{1}\right)$ is called the "reproducing kernel" of the Hilbert space. It has a number of properties usually associated with the (more singular) "delta-function" $\delta\left(x_{2}-x_{1}\right)$ for other spaces of functions!

In the Hilbert space (402) a representation of the Lie algebra (237) is given by

$$
\tilde{K}_{0}=z \frac{d}{d z}+k, \quad \tilde{K}_{+}=z, \quad \tilde{K}_{-}=2 k \frac{d}{d z}+z \frac{d^{2}}{d z^{2}}
$$

\section{Perelomov holomorphic functions}

The corresponding relations for the states $|k, \lambda\rangle$ are

$$
\begin{aligned}
\left(f_{2}, f_{1}\right)_{k, \lambda} & \equiv \int_{\mathbb{D}} d \tilde{\mu}_{k}(\lambda) f_{2}^{*}(\lambda) f_{1}(\lambda) \\
d \tilde{\mu}_{k}(\lambda) & =\frac{2 k-1}{\pi}\left(1-|\lambda|^{2}\right)^{2 k-2}|\lambda| d|\lambda| d \theta \\
\left(\tilde{e}_{k, n_{2}}, \tilde{e}_{k, n_{1}}\right)_{k, \lambda}=\delta_{n_{2} n_{1}}, \quad \tilde{e}_{k, n}(\lambda) & =\sqrt{\frac{(2 k)_{n}}{n !}} \lambda^{n}
\end{aligned}
$$




$$
\begin{aligned}
\Delta_{k}\left(\lambda_{2}^{*}, \lambda_{1}\right)=\sum_{n=0}^{\infty} \tilde{e}_{k, n}^{*}\left(z_{2}\right) \tilde{e}_{k, n}\left(z_{1}\right) & =\left(1-\lambda_{2}^{*} \lambda_{1}\right)^{-2 k}, \\
\int_{\mathbb{D}} d \tilde{\mu}_{k}\left(\lambda_{2}\right) \Delta_{k}\left(\lambda_{2}^{*}, \lambda_{1}\right) \tilde{e}_{k, n}\left(\lambda_{2}\right) & =\tilde{e}_{k, n}\left(\lambda_{1}\right), \\
\int_{\mathbb{D}} d \tilde{\mu}_{k}\left(\lambda_{2}\right) \Delta_{k}\left(\lambda_{2}^{*}, \lambda_{1}\right) f\left(\lambda_{2}\right) & =f\left(\lambda_{1}\right), f(\lambda)=\sum_{n=0}^{\infty} b_{n} \lambda^{n}, \\
\int_{\mathbb{D}} d \tilde{\mu}_{k}(\lambda) \Delta_{k}\left(\lambda_{2}^{*}, \lambda\right) \Delta_{k}\left(\lambda^{*}, \lambda_{1}\right) & =\Delta_{k}\left(\lambda_{2}^{*}, \lambda_{1}\right), \\
\left(f_{2}, f_{1}\right)_{k, \lambda}=\sum_{n=0}^{\infty} \frac{n !}{(2 k)_{n}} b_{n, 2}^{*} b_{n, 1}, & f_{j}(\lambda)=\sum_{n=0}^{\infty} b_{n, j} \lambda^{n}, j=1,2 .
\end{aligned}
$$

As [81]

$$
\int_{0}^{1} d|\lambda|^{2}\left(1-|\lambda|^{2}\right)^{2 k-2}|\lambda|^{2 n+1}=\frac{\Gamma(2 k-1) n !}{\Gamma(2 k+n)},
$$

the factor $2 k-1$ in the measure (410) is multiplied by $\Gamma(2 k-1)$, yielding $\Gamma(2 k)$, which means that the integral and sums (410) - (415) are well-defined for $k>0$. The right-hand side of Eq. (416) may be used in oder to define the scalar product for all $k>0$. The properties (413) - (415) can be interpreted as the completeness relation for the functions (411) where $k>0$.

In the Hilbert space (410) one has the following representation of the Lie algebra (237) by self-adjoint operators

$$
\tilde{K}_{0}=\lambda \frac{d}{d \lambda}+k, \quad \tilde{K}_{+}=2 k \lambda+\lambda^{2} \frac{d}{d \lambda}, \quad \tilde{K}_{-}=\frac{d}{d \lambda} .
$$

\section{Bargmann-Segal holomorphic functions}

The Hilbert space of holomorphic functions associated with the Schrödinger-Glauber coherent states (317) was thoroughly discussed by Bargmann [82]. About the same time such Hilbert spaces were also introduced by Segal into quantum field theory [83] Such a Hilbert space has the following essential properties:

$$
\begin{aligned}
\left(f_{2}, f_{1}\right)_{\alpha} & \equiv \int_{\mathbb{C}} d \tilde{\mu}(\alpha) f_{2}^{*}(\alpha) f_{1}(\alpha), \\
d \tilde{\mu}(\alpha) & =\frac{d^{2} \alpha}{\pi} e^{-|\alpha|^{2}}, \\
\tilde{h}_{n}(\alpha) & =\frac{\alpha^{n}}{\sqrt{n !}}, \quad\left(\tilde{h}_{n_{2}}, \tilde{h}_{n_{1}}\right)_{\alpha}=\delta_{n_{2}, n_{1}}, \\
\Delta\left(\alpha_{2}^{*}, \alpha_{1}\right)=\sum_{n=0}^{\infty} \tilde{h}_{n}^{*}\left(\alpha_{2}\right) \tilde{h}_{n}\left(\alpha_{1}\right) & =e^{\alpha_{2}^{*} \alpha_{1}}, \\
\int_{\mathbb{C}} d \tilde{\mu}\left(\alpha_{2}\right) \Delta\left(\alpha_{2}^{*}, \alpha_{1}\right) \tilde{h}_{n}\left(\alpha_{2}\right) & =\tilde{h}_{n}\left(\alpha_{1}\right), \\
\int_{\mathbb{C}} d \tilde{\mu}\left(\alpha_{2}\right) \Delta\left(\alpha_{2}^{*}, \alpha_{1}\right) f\left(\alpha_{2}\right) & =f\left(\alpha_{1}\right), f(\alpha)=\sum_{n=0}^{\infty} c_{n} \alpha^{n},
\end{aligned}
$$




$$
\begin{aligned}
\int_{\mathbb{C}} d \tilde{\mu}(\alpha) \Delta\left(\alpha_{2}^{*}, \alpha\right) \Delta\left(\alpha^{*}, \alpha_{1}\right)= & \Delta\left(\alpha_{2}^{*}, \alpha_{1}\right), \\
\left(f_{2}, f_{1}\right)_{\alpha}=\sum_{n=0}^{\infty} n ! c_{n, 1}^{*} c_{n, 2}, \quad & f_{j}(\alpha)=\sum_{n=0}^{\infty} c_{n, j} \alpha^{n}, j=1,2 .
\end{aligned}
$$

Recall that $d f^{*}(\alpha) / d \alpha=d f\left(\alpha^{*}\right) / d \alpha=0$ for a holomorphic function $f(\alpha)$.

The mutual adjoint annihilation and creation operators in the Hilbert space (419) are $[82,84]$

$$
a=\frac{d}{d \alpha}, \quad a^{\dagger}=\alpha, \quad\left[a, a^{\dagger}\right]=1 .
$$

Inverting the relations (263) yields the following generators for the Lie algebra (237)

$$
\tilde{K}_{0}=N+k, \quad \tilde{K}_{+}=\alpha \sqrt{N+2 k}, \quad \tilde{K}_{-}=\sqrt{N+2 k} \frac{d}{d \alpha}, \quad N=\alpha \frac{d}{d \alpha} .
$$

\subsubsection{Probabilities for transitions to number states}

\section{Barut-Girardello states}

From the expansions (314) - (317) one immediately can read off the following transition probabilities

$$
p_{k}(n \leftrightarrow z)=\frac{|z|^{2 n}}{(2 k)_{n} n ! g_{k}\left(|z|^{2}\right)}, \quad p_{k}(n=0 \leftrightarrow z)=\frac{1}{g_{k}\left(|z|^{2}\right)} .
$$

In applications one would like to express $|z|$ in terms of the average number $\bar{n}_{k, z}$, here given by Eq. (322). As the ratio $\rho_{k}(|z|)$ depends on $|z|$, too, the inversion $|z|=|z|\left(\bar{n}_{k, z}\right)$ is not immediate. But for large $|z|$ one has in leading order [71]

$$
\rho_{k}(|z|) \rightarrow 1, \quad g_{k}\left(|z|^{2}\right) \rightarrow \frac{\Gamma(2 k)}{2 \sqrt{\pi}} \frac{e^{2|z|}}{|z|^{2 k-1 / 2}} \text { for }|z| \rightarrow \infty,
$$

so that asymptotically

$$
p_{k}(n \leftrightarrow z) \asymp \frac{2 \sqrt{\pi}}{n ! \Gamma(2 k+n)}\left(\bar{n}_{k, z}\right)^{2(n+k-1 / 4)} e^{-2 \bar{n}_{k, z}} .
$$

As the Barut-Girardello states have not yet been produced in a laboratory the distribution (430) has not been tested experimentally (to the best of my knowledge)!

\section{Perelomov states}

Here we get

$$
p_{k}(n \leftrightarrow \lambda)=\left(1-|\lambda|^{2}\right)^{2 k} \frac{(2 k)_{n}}{n !}|\lambda|^{2 n}, \quad p_{k}(n=0 \leftrightarrow \lambda)=\left(1-|\lambda|^{2}\right)^{2 k} .
$$

Using the first of the relations (331) we can also write

$$
p_{k}(n \leftrightarrow \lambda)=\frac{2 k(2 k)_{n}}{\left(\bar{n}_{k, \lambda}+2 k\right) n !}\left(\frac{\bar{n}_{k, \lambda}}{\bar{n}_{k, \lambda}+2 k}\right)^{n} .
$$

As the Perelomov states for $k=1 / 2$ can be produced in the laboratory (see the next subsec.), the distribution (432) has been verified experimentally by counting photon numbers emanating from a Perelomov (squeezed) state [85]. 


\section{Schrödinger-Glauber states}

Here we have the usual Poisson distribution

$$
p_{k}(n \leftrightarrow \alpha)=\frac{|\alpha|^{2 n}}{n !} e^{-|\alpha|^{2}}, \quad|\alpha|^{2}=\bar{n}_{\alpha}
$$

As to its experimental verification see subsec. 6.2.1 above.

\subsection{Physical dynamics described by the basic operators $\tilde{K}_{0}, \tilde{K}_{+}$and $\tilde{K}_{-}$}

The conventional annihilation and creation operators $a$ and $a^{\dagger}$ are a convenient and popular tool in order to build Hamiltonians which describe interactions between elementary excitations, particles and modes, be it scattering, annihilation or creation of them. Completely similar one can construct physically useful model Hamiltonians from the three basic operators $\tilde{K}_{0}, \tilde{K}_{+}$and $\tilde{K}_{-}$(or $\tilde{K}_{1}$ and $\left.\tilde{K}_{2}\right)$.

Actually there are already quite a number of such models in use, especially in the field of quantum optics. They usually come in a form in which the $\tilde{K}_{j}$ are expressed in terms of one or several pairs of $a$ and $a^{\dagger}$. I shall list several typical examples, without any claim of even partial completeness. I shall merely mention explicitly some quite early and some very recent original papers, but otherwise refer to the corresponding chapters in textbooks [86] and their associated References.

An early review on the dynamics of models expressed in terms of the generators $\tilde{K}_{j}$ is Ref. [87]. Early papers using that Lie algebra explicitly for the generation of squeezed states are Refs. [88]. Usually all those applications are discussed in the language of the group $S U(1,1)$. I have stressed in the Introduction and in sec. 3 why the language of the isomorphic symplectic group $S p(2, \mathbb{R})$ is more appropriate because of its potential for generalizations to higher dimensions.

An essential model to start with is the one which we encountered in the context of the unitary transformation (363) which generates the self-adjoint interaction

$$
(\sinh |w|) W(\theta, \vec{K})=(1 / 2)(\sinh |w|)\left(e^{-i \theta} \tilde{K}_{+}+e^{i \theta} \tilde{K}_{-}\right)=(\sinh |w|)\left(\vec{n} \cdot \vec{K}_{\perp}\right)
$$

of Eqs. (364) and (369). The angle $\theta$ here plays the role of a mixing angle as to the operators $\tilde{K}_{1}$ and $\tilde{K}_{2}$ : For $\theta=0$ the term (434) is pure $\tilde{K}_{1}$ and for $\theta=\pi / 2$ pure $\tilde{K}_{2}$. (As to properties of the classical mechanics counterpart of these interactions see subsec. 2.3)

\subsubsection{Generation of Perelomov coherent states}

As the operator $U_{P}$ from Eq. (363) generates the Perelomov coherent states $|k, \lambda\rangle$ from the ground state, the interaction (434) can be used to generate such states experimentally!

In applications the operator $W$ from Eq. (434) is generally multiplied by a "classical" function

$G[g(\tilde{t}), C(\tilde{t}, a)]$, containing coupling constants $g(\tilde{t})$ (possibly time-dependent) and (possibly) time-dependent external "classical" fields $C(\tilde{t}, a)$ which themselves may depend on additional parameters $a$, e.g. second-order or third-order non-linear susceptibilities $\left(\chi^{(2)}\right.$ or $\left.\chi^{(3)}\right)$ [89], spatial coordinates etc.

The potential

$$
V=G[g(t), C(t, a)] W(\theta, \overrightarrow{\tilde{K}})
$$


is then being dealt with in the interaction picture, where $V$ determines the time evolution of the states and the free Hamiltonian $\hbar \omega \tilde{K}_{0}$ that of the operators.

The interaction Hamiltonian (434) is linear in the operators $K_{j}$. Another possibility is to have interactions which are bilinear in the operators $K_{j}$, e.g. proportional to $K_{+} K_{-}$in the description of scattering processes (see below). These can be diagonalized with the help of the Casimir relations (239).

\subsubsection{One-mode generated Lie algebra $\mathfrak{s o}(1,2)$}

Already in section 3.5 we encountered the one-mode representations

$$
\tilde{K}_{0}=\frac{1}{4}\left(2 a^{\dagger} a+1\right), \quad \tilde{K}_{+}=\frac{1}{2} a^{\dagger^{2}}, \quad \tilde{K}_{-}=\frac{1}{2} a^{2}, \quad \tilde{K}_{1}=\frac{1}{4}\left(a^{\dagger^{2}}+a^{2}\right), \quad \tilde{K}_{2}=\frac{1}{4 i}\left(a^{\dagger^{2}}-a^{2}\right) .
$$

Inserted into Eq. (434) the term $W$ describes the creation or annihilation of two identical modes (photons).

\section{Degenerate parametric down-conversions and amplifications}

Such processes occur experimenally in so-called "degenerate parametric down-conversions" where a classical electromagnetic ("pump") wave of frequency $2 \omega$ generates two identical photons each with frequency $\omega$ in a $\chi^{(2)}$ nonlinear medium and amplifying one of the "quadratures" $\left(a+a^{\dagger}\right)$ and $i\left(a^{\dagger}-a\right)$ and reducing the other. Thus, in applications one often chooses $\theta=\pi / 2$ in Eq. (435) in order to generate squeezed light (cf. Eq. (170) ).

\section{Squared hermitian amplitudes}

The square of the hermitian field mode

$$
E=\lambda\left(a e^{-i \omega t}+a^{\dagger} e^{i \omega t}\right), \lambda \in \mathbb{R},
$$

may be written in terms of the operators (436) as

$$
E^{2}=4 \lambda^{2}\left[\tilde{K}_{0}+\tilde{K}_{1} \cos (2 \omega t)-\tilde{K}_{2} \sin (2 \omega t)\right]
$$

This expression has also been used for the generation of squeezed light [90].

\subsubsection{Interactions bilinear in the $K_{j}$}

\section{Optical Kerr effect}

In some materials a light beam has an additional term in its refractive index which is proportional to the intensity of the light [91], i.e. that extra part of the index is proportional to the square of the electric field. Phenomenologically this means that the polarization of the material is proportional to the 3 rd power of the electric field, with a nonlinear coefficient $\chi^{(3)}$. A very simple quantum mechanical model for the associated elementary process is given by the interaction term

$$
g \chi^{(3)} a^{\dagger} N a=g \chi^{(3)} a^{\dagger} a^{\dagger} a a \propto \chi^{(3)} \tilde{K}_{+} \tilde{K}_{-},
$$

where, according to Eqs. (239) and (148), the product $\tilde{K}_{+} \tilde{K}_{-}$can be replaced by $\tilde{K}_{0}\left(\tilde{K}_{0}-\right.$ $1)+(3 / 16) 1$. Thus, the total Hamiltonian can be diagonalized in terms of the number states $|k, n\rangle$, where $k=1 / 4$ and $=3 / 4$. 


\section{Degenerate four-wave mixing}

The simple model interaction Hamiltonian (439) may also be used in order to describe another optical process in a non-linear medium with 3rd order susceptibility: Two high intensity classical optical light beams of the same frequency $\omega$ interact with a weak (quantum) beam with frequency $\omega$, creating a fourth photon beam, again with the same frequency $\omega$ and special properties of interest, e.g. squeezed light. The process, and the corresponding "nondegenerate one" mentioned below, is called "four-wave mixing" and played a prominent role in the first stages of light squeezing [92]. The annihilation and creation of two photons are represented by the operators $a$ and $a^{\dagger}$.

\subsubsection{Two-mode generated Lie algebra $\mathfrak{s o}(1,2)$}

A much larger variety of unitary irreducible representations can be generated with two "canonical" annihilation and creation operators [93]:

$$
\tilde{K}_{+}=a_{1}^{\dagger} a_{2}^{\dagger}, \tilde{K}_{-}=a_{1} a_{2}, \tilde{K}_{0}=\frac{1}{2}\left(a_{1}^{\dagger} a_{1}+a_{2}^{\dagger} a_{2}+1\right)
$$

obey the commutation relations (237).

The tensor product $\mathcal{H}_{1}^{\text {osc }} \otimes \mathcal{H}_{2}^{\text {osc }}$ of the two harmonic oscillator Hilbert spaces contains all the irreducible unitary representations of the group $S U(1,1) \cong S L(2, \mathbb{R})=S p(2, \mathbb{R})$ (for which $k=1 / 2,1,3 / 2, \ldots)$ in the following way:

Let $\left|n_{j}\right\rangle_{j}, n_{j}=0,1, \ldots, j=1,2$, be the eigenstates of the number operators $N_{j}=a_{j}^{\dagger} a_{j}$, generated by $a_{j}^{\dagger}$ from the oscillator ground states.

Then each of those two subspaces of $\mathcal{H}_{1}^{\text {osc }} \otimes \mathcal{H}_{2}^{\text {osc }}=\left\{\left|n_{1}\right\rangle_{1} \otimes\left|n_{2}\right\rangle_{2}\right\}$ with fixed $\left|n_{1}-n_{2}\right| \neq 0$ contains an irreducible representation with Bargmann index

$$
k=1 / 2+\left|n_{1}-n_{2}\right| / 2=1,3 / 2,2, \ldots,
$$

i.e. the operator $N_{1}-N_{2}$ commutes with all 3 operators in Eqs. (440)

The number $n$ in the eigenvalue $n+k$ of $\tilde{K}_{0}$ is given by

$$
n=\min \left\{n_{1}, n_{2}\right\}(=0,1,2, \ldots) .
$$

For the "diagonal" case $n_{2}=n_{1}$ one gets the unitary representation with $k=1 / 2$.

Inserting the operators (440) into the interaction (435) yields other examples of associated physical processes:

\section{Nondegenerate parametric down conversion and amplification}

In analogy to the degenerate case mentioned above here a classical light beam of frequency $2 \omega$ generates two photons of now different frequencies $\omega_{1}$ and $\omega_{2}$ with $2 \omega=\omega_{1}+\omega_{2}$ in a nonlinear medium.

\section{Nondegenerate four-wave mixing}

Here the frequencies of the two pump beams and those of the photons are no longer equal. Now the operators $K_{+}$and $K_{-}$in the effective Hamiltonian (439) are replaced by those of Eq. (440). 


\section{Mach-Zehnder interferometer}

The group $S U(1.1) \cong S p(2, \mathbb{R})$ has played a prominent role in the quantum optical descriptions of the venerable Mach-Zehnder interferometer [94].

\subsubsection{Generation of Barut-Girardello coherent states}

Contrary to the Perelomov coherent states the Barut-Girardello coherent states have not yet produced in the laboratory (to the best of my knowledge!). There exist, however, a number of proposals how to generate them [95]. One problem is the the lack of a unitary operator analogously to Eq. (363) as already discussed in subsecs. 6.2.3 and 6.2.4.

\subsubsection{Holstein-Primakoff type generators}

The one-mode and the 2-mode versions of the generators $\tilde{K}_{0}, \tilde{K}_{+}$and $\tilde{K}_{-}$from above can only produce representations with $k=1 / 4,3 / 4$ and $k=1 / 2,1,3 / 2, \ldots$. As we are especially interested in representations with small $k<1 / 4$ we have to use corresponding representations. Some of them will be discussed in the next Section. If one wants to construct those with the help of the usual annihilation and creation operators one can try the nonlinear HolsteinPrimakoff-type operators [27]

$$
\tilde{K}_{0}=N+k, \quad \tilde{K}_{+}=a^{\dagger} \sqrt{N+2 k} \quad \tilde{K}_{-}=\sqrt{N+2 k} a, \quad N=a^{\dagger} a .
$$

Inserted into the interaction term (434) and (435) one has to find experimental ways in order to generate a ground state with $k \neq 1 / 2$ (see also subsec. 9.1) and to implement the nonlinear factor $\sqrt{N+2 k}[96]$.

\subsubsection{Additional proposals for using symplectic groups in quantum optics}

There have been a number of papers with proposals to use symplectic groups $S p(2 n, \mathbb{R}), n>$ 1 , in quantum optics which are merely quoted here [97].

\section{$7 \quad$ Examples of explicit Hilbert spaces for the $(\varphi, \mathrm{I})$-model of the harmonic oscillator}

\subsection{The case $k=1 / 2$}

As a first step let us discuss the well-known quantum mechanics of the HO in the framework of concrete irreducible unitary representations of the group $S p(2, \mathbb{R})$ with Bargmann index $k=1 / 2$ [98], before passing to the more general case with $k \neq 1 / 2$ :

\subsubsection{The Hardy space $H_{+}^{2}$ on the circle as the Hilbert space for the HO}

The simplest example is the "Hardy (sub)space" $H_{+}^{2}\left(S^{1}, d \vartheta\right)$ of the usual Hilbert space $L^{2}\left(S^{1}, d \vartheta\right)$ on the unit circle $S^{1}$ with the scalar product

$$
\left(f_{2}, f_{1}\right)=\frac{1}{2 \pi} \int_{S^{1}} d \vartheta f_{2}^{*}(\vartheta) f_{1}(\vartheta),
$$


and the orthonormal basis

$$
e^{i n \vartheta}, \quad n \in \mathbb{Z}
$$

The associated Hardy space $H_{+}^{2}\left(S^{1}, d \vartheta\right)$ is spanned by the basis consisting of the elements with non-negative $n$, namely

$$
e_{n}(\vartheta)=e^{i n \vartheta}, \quad n=0,1,2, \cdots
$$

If we have two Fourier series $\in H_{+}^{2}\left(S^{1}, d \vartheta\right)$,

$$
f_{1}(\vartheta)=\sum_{n=0}^{\infty} a_{n} e^{i n \vartheta}, \quad f_{2}(\vartheta)=\sum_{n=0}^{\infty} b_{n} e^{i n \vartheta}
$$

they have the scalar product

$$
\left(f_{2}, f_{1}\right)_{+}=\frac{1}{2 \pi} \int_{S^{1}} d \vartheta f_{2}^{*}(\vartheta) f_{1}(\vartheta)=\sum_{n=0}^{\infty} b_{n}^{*} a_{n} .
$$

The reproducing kernel here has the form

$$
\Delta\left(\varphi_{2}, \varphi_{1}\right)=\sum_{n=0}^{\infty} e_{n}\left(\varphi_{2}\right)^{*} e_{n}\left(\varphi_{1}\right)=\left(1-e^{i\left(\varphi_{1}-\varphi_{2}\right)}\right)^{-1}
$$

with the usual property

$$
\frac{1}{2 \pi} \int_{0}^{2 \pi} d \varphi_{2} \Delta\left(\varphi_{2}, \varphi_{1}\right) e_{n}\left(\varphi_{2}\right)=e_{n}\left(\varphi_{1}\right)
$$

The kernel has a singularity (pole) for $\varphi_{2}=\varphi_{1}$. In calculations one has to replace $\exp \left(i\left(\varphi_{1}-\varphi_{2}\right)\right)$ by $(1-\epsilon) \exp \left(i\left(\varphi_{1}-\varphi_{2}\right)\right)$ and then take the limit $\epsilon \rightarrow 0$ at the end.

The $S p(2, \mathbb{R})$ Lie algebra generators for $k=1 / 2$ are

$$
\begin{aligned}
\tilde{K}_{0} & =\frac{1}{i} \partial_{\vartheta}+\frac{1}{2} \\
\tilde{K}_{+} & =e^{i \vartheta}\left(\frac{1}{i} \partial_{\vartheta}+1\right)=e^{i \vartheta}\left(\tilde{K}_{0}+\frac{1}{2}\right) \\
\tilde{K}_{-} & =e^{-i \vartheta} \frac{1}{i} \partial_{\vartheta}=e^{-i \vartheta}\left(\tilde{K}_{0}-\frac{1}{2}\right)=\left(\frac{1}{i} \partial_{\vartheta}+1\right) e^{-i \vartheta} .
\end{aligned}
$$

The right-hand side of the scalar product (448) coincides with the right-hand side of the scalar product (416) for $k=1 / 2$. Actually the functions (446) of the present Hilbert space $H_{+}^{2}\left(S^{1}, d \vartheta\right)$ may be considered as limits of those from Eq. (411) with $k=1 / 2$ for $|\lambda| \rightarrow 1$ : For $\lambda=|\lambda| \exp (i \vartheta)$ the operators (418) become the operators (451) $-(453)$ in the limit $|\lambda| \rightarrow 1$.

For the operators (451) - (453) the relations (249) - (251) take the form

$$
\begin{aligned}
\tilde{K}_{0} e_{n}(\vartheta) & =\left(n+\frac{1}{2}\right) e_{n}(\vartheta), \\
\tilde{K}_{+} e_{n}(\vartheta) & =(n+1) e_{n+1}(\vartheta) \\
\tilde{K}_{-} e_{n}(\vartheta) & =n e_{n-1}(\vartheta) .
\end{aligned}
$$


The (dimensionless) Hamilton operator for the $(\varphi, I)$-model of the HO now has the extremely simple explicit form

$$
\tilde{H}(\vec{K})=\tilde{K}_{0}=\frac{1}{i} \partial_{\vartheta}+\frac{1}{2},
$$

and the corresponding simple eigenfunctions (446)!

I would like to stress again (like I did in Refs. [13] and [25]) that the mathematical variable $\vartheta$ used here is not the canonically conjugate "observable" of the operator (457): the angle $\vartheta$ is not a self-adjoint multiplication operator nor is $\exp (i \vartheta)$ a unitary operator! The self-adjoint observables "conjugate" to $\tilde{K}_{0}$ are the operators $\tilde{K}_{1}$ and $\tilde{K}_{2}$ !

The composite ladder operators

$$
\begin{aligned}
A & =\left(\tilde{K}_{0}+1 / 2\right)^{-1 / 2} \tilde{K}_{-}=\tilde{K}_{-}\left(\tilde{K}_{0}-1 / 2\right)^{-1 / 2}=e^{-i \vartheta}\left(\tilde{K}_{0}-1 / 2\right)^{1 / 2} \\
A^{\dagger} & =\tilde{K}_{+}\left(\tilde{K}_{0}+1 / 2\right)^{-1 / 2}=e^{i \vartheta}\left(\tilde{K}_{0}+1 / 2\right)^{1 / 2}
\end{aligned}
$$

have the desired properties

$$
A e_{n}(\vartheta)=\sqrt{n} e_{n-1}(\vartheta), \quad A^{\dagger} e_{n}(\vartheta)=\sqrt{n+1} e_{n+1}(\vartheta)
$$

and, therefore, have the usual matrix elements [99]. The same applies, of course, to those of the composite operators $\tilde{Q}$ and $\tilde{P}$ :

$$
\tilde{Q}=\frac{1}{\sqrt{2}}\left(A^{\dagger}+A\right), \quad \tilde{P}=\frac{i}{\sqrt{2}}\left(A^{\dagger}-A\right) .
$$

Obviously we can reproduce all the quantum physical properties of the HO which - over decades - have been derived by means of the operators $\tilde{Q}$ and $\tilde{P}$ and the $(\tilde{q}, \tilde{p})$-Hamiltonian (271).

The (composite) number operator $N=A^{\dagger} A$ is as expected:

$$
N=A^{\dagger} A=\tilde{K}_{+}\left(\tilde{K}_{0}+1 / 2\right)^{-1} \tilde{K}_{-}=e^{i \vartheta}\left(\tilde{K}_{0}+1 / 2\right)\left(\tilde{K}_{0}+1 / 2\right)^{-1} e^{-i \vartheta}\left(\tilde{K}_{0}-1 / 2\right)=\frac{1}{i} \partial_{\vartheta} .
$$

Remarks:

- The eigenfunctions (446) are periodic:

$$
e_{n}(\vartheta+2 \pi)=e_{n}(\vartheta)
$$

Further below we shall encounter unitarily equivalent quasi-periodic eigenfunctions.

- The ground state of the Hamiltonian (457) is given by the number 1 :

$$
e_{n=0}(\vartheta)=1
$$

- The probability densities $p_{n}(\vartheta)$ associated with the "number states" (446) are completely flat:

$$
p_{n}(\vartheta)=1, n=0,1, \cdots .
$$

- The number state relations (289) - (294) for general $k$ do, of course, hold in the present special case $k=1 / 2$, too! 
The time-dependent Schrödinger equation for a general state $\psi(\tilde{t}, \vartheta)$ is given by

$$
i \partial_{\tilde{t}} \psi(\tilde{t}, \vartheta)=\tilde{K}_{0} \psi(\tilde{t}, \vartheta),
$$

which means that the eigenfunctions (446) have the time dependence

$$
e_{n}(\tilde{t}, \vartheta)=e^{-i \tilde{E}_{n} \tilde{t}} e_{n}(\vartheta)=e^{-i \tilde{t} / 2} e^{i n(\vartheta-\tilde{t})}, \tilde{E}_{n}=n+1 / 2
$$

and $\psi(\tilde{t}, \vartheta)$ may be expanded as

$$
\psi(\tilde{t}, \vartheta)=e^{-i \tilde{t} / 2} \sum_{n=0}^{\infty} c_{n} e^{i n(\vartheta-\tilde{t})}, \quad c_{n}=\left(e_{n}, \psi(\tilde{t}=0)\right)_{+}
$$

The last two equations show again that the angle $\vartheta$ plays the role of a time variable (up to a sign) and that the circle $S^{1}$ parametrized by $\vartheta \in \mathbb{R} \bmod 2 \pi$ becomes "unwrapped" onto the time-axis, finitely or infinitely many times, thus realizing an $m$-fold or a universal covering of the circle or of the group $U(1)$ !

Introducing the usual quantities with physical dimensions, we get from Eqs. (40) - (43)

$$
\begin{aligned}
H & =\hbar \omega \tilde{K}_{0}, H e_{n}(\vartheta)=E_{n} e_{n}(\vartheta), \\
E_{n} & =\hbar \omega \tilde{E}_{n}=\hbar \omega(n+1 / 2), \\
i \hbar \partial_{t} \psi(t, \vartheta) & =H \psi(t, \vartheta), \\
e_{n}(t, \vartheta) & =e^{-i\left(E_{n} / \hbar\right) t} e^{i n \vartheta}=e^{-i \omega t / 2} e^{i n(\vartheta-\omega t)} .
\end{aligned}
$$

\subsubsection{Space reflections and time reversal}

According to Subsects. 4.5 and 5.1 we can implement the space reflections $\Pi$ and the time reversal $T$ as follows:

$$
\Pi: \quad \vartheta \rightarrow \vartheta \pm \pi
$$

which implies

$$
\begin{aligned}
\partial_{\vartheta} & \rightarrow \partial_{\vartheta}, \\
\tilde{K}_{0} & \rightarrow \tilde{K}_{0}, \\
K_{ \pm} & \rightarrow-K_{ \pm}, \\
\tilde{Q} & \rightarrow-\tilde{Q}, \\
\tilde{P} & \rightarrow-\tilde{P}, \\
e_{n}(\vartheta) & \rightarrow e_{n}(\vartheta \pm \pi)=(-1)^{n} e_{n}(\vartheta) .
\end{aligned}
$$

The last relation shows that the functions $e_{n}(\vartheta)$ have the same symmetry properties under reflections as the usual Hermite functions (150).

Furthermore

$$
T: \vartheta \rightarrow-\vartheta, \quad i \rightarrow-i
$$


yielding

$$
\begin{aligned}
\frac{1}{i} \partial_{\vartheta} & \rightarrow \frac{1}{i} \partial_{\vartheta}, \\
\tilde{K}_{0} & \rightarrow \tilde{K}_{0}, \\
K_{ \pm} & \rightarrow K_{ \pm}, \\
A, A^{\dagger} & \rightarrow A, A^{\dagger}, \\
\tilde{Q} & \rightarrow \tilde{Q}, \\
\tilde{P} & \rightarrow-\tilde{P}, \\
e_{n}(\vartheta) & \rightarrow\left[e_{n}(-\vartheta)\right]^{*}=e_{n}(\vartheta) .
\end{aligned}
$$

\subsubsection{Perturbations}

Like in the classical case (Eqs. (63) - (65) ) external time-dependent perturbations of the Hamilton operator (457) can be integrated immediately: Take

$$
\tilde{H}=\tilde{K}_{0}+f(\tilde{t})
$$

where $f(\tilde{t})$ is a given real function of time. Then the usual product separation of variables gives the following solution of the time-dependent Schrödinger Eq.

$$
i \partial_{\tilde{t}} \psi(\tilde{t}, \vartheta)=\left[\tilde{K}_{0}+f(\tilde{t})\right] \psi(\tilde{t}, \vartheta)
$$

The ansatz

$$
\psi(\tilde{t}, \vartheta)=v(\tilde{t}) u(\vartheta)
$$

yields

$$
\left\{i\left[\partial_{\tilde{t}} v(\tilde{t})\right] / v(\tilde{t})\right\}-f(\tilde{t})=\left\{\frac{1}{i}\left[\partial_{\vartheta} u(\vartheta)\right] / u(\vartheta)\right\}+\frac{1}{2}=\tilde{E}=\text { const. },
$$

with the (normalized) solution

$$
v(\tilde{t})=e^{-i\left[\tilde{E} \tilde{t}+\int_{0}^{\tilde{t}} d \tau f(\tau)\right]}
$$

For $u(\vartheta)$ we can take

$$
u(\vartheta)=e_{n}(\vartheta), \text { with } \tilde{E}=n+1 / 2,
$$

or appropriate superpositions.

Thus, the perturbation $f(\tilde{t})$ causes a time-dependent modification of the phase $\tilde{E} \tilde{t}$. If

$$
f(\tilde{t})=a=\text { const. }
$$

then we have

$$
v(\tilde{t})=e^{-i(\tilde{E}+a) \tilde{t}},
$$

i.e. we have introduced an effective (dynamical) $k \neq 1 / 2$ ! For an explicit example see subsec. 9.1 .

For the periodic perturbation

$$
f(\tilde{t})=\epsilon \cos (\tilde{\sigma} \tilde{t})
$$


we get for $v(\tilde{t})$ the time - dependent phase factor

$$
v(\tilde{t})=e^{-i[\tilde{E} \tilde{t}+(\epsilon / \tilde{\sigma}) \sin (\tilde{\sigma} \tilde{t})]} .
$$

Similarly we have for the slightly different perturbation

$$
\tilde{K}_{0} \rightarrow[1+g(\tilde{t})] \tilde{K}_{0}
$$

a corresponding phase factor

$$
e^{-i \tilde{E} \tilde{t}} \rightarrow e^{-i \tilde{E}\left[\tilde{t}+\int_{0}^{\tilde{t}} d \tau g(\tau)\right]} .
$$

If one inserts for $g(\tilde{t})$ the same expressions as for $f(\tilde{t})$ in Eqs. (494) and (496) one gets the corresponding similar expressions for the phase factor (499).

\subsubsection{A unitary transformation}

The following unitary transformation is of interest, especially later for the more general case $k \neq 1 / 2$ :

In the above description of the states (524) and the operators (451) - (453) the dependence on the index $k=1 / 2$ is contained in the operators. We shall see below that in the general case we have

$$
\tilde{K}_{0}=\frac{1}{i} \partial_{\vartheta}+k, \tilde{K}_{+}=e^{i \vartheta}\left(\frac{1}{i} \partial_{\vartheta}+2 k\right), \tilde{K}_{-}=e^{-i \vartheta} \frac{1}{i} \partial_{\vartheta} .
$$

The unitary transformation in question is defined by the replacement

$$
e_{n}(\vartheta)=e^{i n \vartheta} \rightarrow e_{1 / 2, n}(\vartheta)=e^{i(n+1 / 2) \vartheta}, n=0,1, \cdots
$$

It shifts the ground state energy characterized by $k=1 / 2$ from the Hamiltonian (457) to the eigenfunctions (446).

The operators (451) - (453) now take the form

$$
\begin{aligned}
\tilde{K}_{0} & =\frac{1}{i} \partial_{\vartheta}, \\
\tilde{K}_{+} & =e^{i \vartheta}\left(\frac{1}{i} \partial_{\vartheta}+1 / 2\right), \\
\tilde{K}_{-} & =e^{-i \vartheta}\left(\frac{1}{i} \partial_{\vartheta}-1 / 2\right) .
\end{aligned}
$$

The eigenfunctions (501) are only quasi-periodic:

$$
e_{1 / 2, n}(\vartheta+2 \pi)=e^{i \pi / 2} e_{1 / 2, n}(\vartheta)
$$

The relations (454) - (461) remain unchanged.

\subsubsection{Coherent state wave functions and their probability densities}

Passing to the $(\varphi, I)$-model of the HO and its associated $S p(2, \mathbb{R})$-structure yields additional information, even for $k=1 / 2$ : 


\section{Wave functions on $S^{1}$}

We have two additional coherent states: Setting $k=1 / 2$ and $|k=1 / 2, n\rangle=e_{n}(\vartheta)$ in Eqs. (314) - (316) the series can be summed immediately, yielding

$$
|k=1 / 2, z\rangle(\vartheta) \equiv f_{z}(\vartheta)=\frac{e^{z e^{i \vartheta}}}{\sqrt{I_{0}(2|z|)}}
$$

and

$$
|k=1 / 2, \lambda\rangle(\vartheta) \equiv f_{\lambda}(\vartheta)=\frac{\left(1-|\lambda|^{2}\right)^{1 / 2}}{1-\lambda e^{i \vartheta}} .
$$

These new coherent state functions have all the properties listed in sec. 6 for general $k$.

The series (317) cannot be summed in an elementary way but yields

$$
\begin{aligned}
|k=1 / 2, \alpha\rangle(\vartheta) & \equiv f_{\alpha}(\vartheta)=e^{-|\alpha|^{2} / 2} \hat{f}_{\alpha}(\vartheta) \\
\hat{f}_{\alpha}(\vartheta) & =\sum_{n=0}^{\infty} \frac{\left(\alpha e^{i \vartheta}\right)^{n}}{\sqrt{n !}}=\sum_{n=0}^{\infty} \frac{\left(|\alpha| e^{i(\vartheta-\beta)}\right)^{n}}{\sqrt{n !}} .
\end{aligned}
$$

The function $\hat{f}_{\alpha}$ in Eq. (509) is an entire function [100] of its complex argument

$$
\zeta=|\alpha| e^{i(\vartheta-\beta)} .
$$

The growth of such functions for large $|\alpha|$ has been investigated for more than a century [100]. Application of standard saddle point methods [101] yields for functions like

$$
f^{(\rho)}(\zeta)=\sum_{n=0}^{\infty} \frac{\zeta^{n}}{(n !)^{1 / \rho}}
$$

the following asymptotic expansion [102]

$$
f^{(\rho)}(\zeta) \asymp \sqrt{\rho}(2 \pi)^{(1-1 / \rho) / 2} \zeta^{(\rho-1) / 2} e^{\zeta^{\rho} / \rho} \text { for }|\zeta| \rightarrow \infty,|\arg (\zeta)| \leq \frac{\pi}{2 \rho}-\epsilon, \epsilon>0 .
$$

For that part of the complex plane where the function (511) decreases with increasing $|\zeta|$, Ref. [103] gives the estimate

$$
f^{(\rho)}(\zeta) \asymp[1-\sin (\pi / \rho) / \pi] \frac{1}{\zeta(\ln \zeta)^{1 / \rho}} \text { for }|\zeta| \rightarrow \infty, \frac{\pi}{2 \rho}+\epsilon \leq|\arg (\zeta)| \leq \pi .
$$

As the assumptions made in Ref. [103] include the exactly known case $\rho=1$ the estimate (513) does not appear to be a good one!

The limits (512) for $\arg (\zeta)$ come from the requirement $\Re\left(\zeta^{\rho}\right)>0$. They also imply $\rho \geq 1 / 2$. The result for the exponential growth in the sector $|\arg (\zeta)| \leq \frac{\pi}{2 \rho}-\epsilon$ shows $f^{(\rho)}(\zeta)$ to be of "order" $\rho$ and of "type" $1 / \rho$ there.

The function $\hat{f}_{\alpha}$ from Eq. (509) has $\rho=2$ and therefore we get for the wave function (508)

$$
f_{\alpha}(\vartheta) \asymp(2 \pi)^{1 / 4} \sqrt{2|\alpha|} e^{i(\vartheta-\beta) / 2} e^{-|\alpha|^{2}\left[1-e^{2 i(\vartheta-\beta)}\right] / 2}, \text { for }|\alpha| \rightarrow \infty,|\vartheta-\beta| \leq \frac{\pi}{4}-\epsilon .
$$




\section{Probability densities}

The probability density of the wave function (506) is given by

$$
p_{z}(\vartheta)=\left|f_{z}(\vartheta)\right|^{2}=\frac{e^{2|z| \cos (\vartheta-\phi)}}{I_{0}(2|z|)} .
$$

For large $|z|$ we have [79]

$$
I_{0}(2|z|) \asymp \frac{e^{2|z|}}{2 \sqrt{\pi|z|}}[1+O(1 /|z|)]
$$

so that

$$
p_{z}(\vartheta) \asymp 2 \sqrt{\pi|z|} e^{-2|z|[1-\cos (\vartheta-\phi)]}[1+O(1 /|z|)] .
$$

For $|\vartheta-\phi| \ll 1$ the density (517) takes (locally) an approximate Gaussian form:

$$
p_{z}(\vartheta) \asymp 2 \sqrt{\pi|z|} e^{-|z|(\vartheta-\phi)^{2}}[1+O(1 /|z|)] \text { for large }|z| \text {. }
$$

The last relation shows that for large $|z|$ (the classical limit) the density $p_{z}(\vartheta)$ has a sharp peak at $\vartheta=\phi=-\arg (z)$, so that in the correspondence limit $|z| \rightarrow \infty$ the variable $\vartheta$ approaches the "classical" angle $\phi$.

As $p_{z}(\vartheta+2 \pi)=p_{z}(\vartheta)$ and $p_{z}(\vartheta)$ an even function of $\vartheta-\phi$ it may be expanded into a Fourier series with respect to $\cos (n \vartheta)$ : Using the relation [104]

$$
\frac{1}{2 \pi} \int_{0}^{2 \pi} d \vartheta e^{2|z| \cos \vartheta} \cos (n \vartheta)=I_{n}(2|z|),
$$

we get

$$
p_{z}(\vartheta)=\frac{1}{I_{0}(2|z|)}\left\{I_{0}(2|z|)+2 \sum_{n=1}^{\infty} I_{n}(2|z|) \cos [n(\vartheta-\phi)]\right\} .
$$

For the probability density of the wave function (507) we have

$$
p_{\lambda}(\vartheta)=\frac{1-|\lambda|^{2}}{1-2|\lambda| \cos (\vartheta-\theta)+|\lambda|^{2}} .
$$

It has the properties

$$
\begin{aligned}
& p_{\lambda}(\vartheta) \rightarrow 1 \text { for }|\lambda| \rightarrow 0, \\
& p_{\lambda}(\vartheta) \approx \frac{\epsilon}{(1-\epsilon)[1-\cos (\vartheta-\theta)]} \text { for }|\lambda|=1-\epsilon, 0<\epsilon \ll 1, \cos (\vartheta-\theta) \neq 1, \\
& p_{\lambda}(\vartheta)=1+2 \sum_{n=1}^{\infty} \cos n(\vartheta-\theta)|\lambda|^{n} .
\end{aligned}
$$

Eq. (523) shows that for $|\lambda| \rightarrow 1^{-}$(the classical limit) $p_{\lambda}(\vartheta)$ is strongly peaked at $\vartheta=\theta$.

For calculating the coefficients of the Fourier series (524) the relation [105]

$$
\int_{0}^{2 \pi} d \vartheta \frac{\cos n \vartheta}{1-2|\lambda| \cos \vartheta+|\lambda|^{2}}=\frac{2 \pi|\lambda|^{n}}{1-|\lambda|^{2}},|\lambda|<1,
$$

has been used. 
The function (521) is well-known in the mathematical literature as the "Poisson kernel" $P_{|\lambda|}(\vartheta-\phi)$ for the representation of harmonic functions inside the unit disc [106] by functions on the boundary $\partial \mathbb{D}=S^{1}$.

The exact probability density $p_{\alpha}(\vartheta)$ for the wave function (508) appears somewhat "unruly":

$$
p_{\alpha}(\vartheta)=\left|f_{\alpha}(\vartheta)\right|^{2}=e^{-|\alpha|^{2}} \sum_{n_{1}, n_{2}=0}^{\infty} \frac{|\alpha|^{n_{1}+n_{2}}}{\sqrt{n_{1} ! n_{2} !}} e^{i(\vartheta-\beta)\left(n_{1}-n_{2}\right)} .
$$

More instructive is the density for the asymptotic expansion (514):

$$
p_{\alpha}(\vartheta) \asymp 2 \sqrt{2 \pi}|\alpha| e^{-|\alpha|^{2}[1-\cos 2(\vartheta-\beta)]} \text { for large }|\alpha|,
$$

which for $|\vartheta-\beta| \ll 1,|\alpha||\vartheta-\beta|$ finite, becomes a Gaussian distribution, too:

$$
p_{\alpha}(\vartheta) \approx 2 \sqrt{2 \pi}|\alpha| e^{-2|\alpha|^{2}(\vartheta-\beta)^{2}} \text { for }|\vartheta-\beta| \ll 1,|\alpha| \rightarrow \infty,|\alpha||\vartheta-\beta| \text { finite } .
$$

As $p_{\alpha}(\vartheta)$ is a periodical and even function of $\vartheta-\beta$ it may be Fourier expanded, but the result does not appear to be very instructive.

\subsubsection{Expectation values and transition probabilities}

All the properties of the 3 types of coherent states listed in sec. 6 for general $k$ do hold, of course, for the special value $k=1 / 2$, too. I, therefore, mention here just a few special features:

We have (cf. Eqs. (315) and (320)

$$
g_{1 / 2}\left(|z|^{2}\right)=I_{0}(2|z|), \rho_{1 / 2}(|z|)=\frac{I_{1}(2|z|)}{I_{0}(2|z|)} .
$$

Remarkable is that now (cf. Eq. (323) )

$$
\left\langle N^{2}\right\rangle_{1 / 2, z}=|z|^{2},
$$

which provides a direct "measurement" of the modulus $|z|$. For the transition probabilities (428) and (431) we get

$$
\begin{gathered}
p(1 / 2, n \leftrightarrow z)=\frac{|z|^{2 n}}{(n !)^{2} I_{0}(2|z|)} \asymp \frac{2 \sqrt{\pi}|z|^{2 n+1 / 2}}{(n !)^{2}} e^{-2|z|} \text { for }|z| \rightarrow \infty . \\
p(1 / 2, n \leftrightarrow \lambda)=\left(1-|\lambda|^{2}\right)|\lambda|^{2 n}=\frac{1}{\bar{n}_{\lambda}+1}\left(\frac{\bar{n}_{\lambda}}{\bar{n}_{\lambda}+1}\right)^{n}, \bar{n}_{\lambda} \equiv \bar{n}_{1 / 2, \lambda} .
\end{gathered}
$$

The last probability may (formally) be interpreted in the context of Bose-Einstein statistics [107]: Assume that a system of free Bose-Einstein quanta has distinct energy levels $E_{\nu}, \nu=$ $0,1, \ldots$ and is in a heat bath with inverse temperature $\beta=1 /\left(k_{B} T\right)$ and chemical potential $\mu$. Then

$$
\left(1-|\lambda|^{2}\right)|\lambda|^{2 n},|\lambda|^{2}=e^{-\beta\left(E_{\nu}-\mu\right)}
$$

is the probability to find $n$ quanta in a state with energy $E_{\nu}$.

As already mentioned previously the distribution (532) has been verified experimentally [85]. 
From Eq. (379) we get

$$
\left(f_{\lambda}, f_{z}\right)_{+}=\frac{\left(1-|\lambda|^{2}\right)^{1 / 2}}{\sqrt{I_{0}(2|z|)}} e^{\lambda^{*} z},\left|\left(f_{\lambda}, f_{z}\right)_{+}\right|^{2}=\frac{1-|\lambda|^{2}}{I_{0}(2|z|)} e^{2|\lambda||z| \cos (\phi-\theta)} .
$$

Furthermore [71]

$$
\begin{aligned}
& \left(f_{\alpha}, f_{z}\right)_{+}=\frac{e^{-|\alpha|^{2} / 2}}{\sqrt{I_{0}(2|z|)}} \sum_{n=0}^{\infty} \frac{\left(\alpha^{*} z\right)^{n}}{(n !)^{3 / 2}} \\
& \left(f_{\alpha}, f_{\lambda}\right)_{+}=e^{-|\alpha|^{2} / 2}\left(1-|\lambda|^{2}\right)^{1 / 2} \sum_{n=0}^{\infty} \frac{\left(\alpha^{*} \lambda\right)^{n}}{\sqrt{n !}} .
\end{aligned}
$$

In evaluating the series (535) and (536) we encounter the same problems as for the series (509). The asymptotic expansion (512) yields for the transition probabilities

$$
\begin{aligned}
\left|\left(f_{\alpha}, f_{z}\right)_{+}\right|^{2} \asymp & \frac{2}{3 \sqrt{2 \pi}|\alpha z|^{1 / 3}} \frac{e^{-|\alpha|^{2}}}{I_{0}(2|z|)} e^{\left.3|\alpha z|^{2 / 3} \cos [2(\beta-\phi) / 3)\right]} \\
& \text { for large }|\alpha z|,|\beta-\phi| \leq 3 \pi / 4-\epsilon, \\
\left|\left(f_{\alpha}, f_{\lambda}\right)_{+}\right|^{2} \asymp & 2 \sqrt{2 \pi}|\lambda \alpha| e^{-|\alpha|^{2}}\left(1-|\lambda|^{2}\right) e^{|\lambda \alpha|^{2} \cos 2(\beta-\theta)} \\
& \text { for large }|\alpha|,|\beta-\theta| \leq \pi / 4-\epsilon .
\end{aligned}
$$

\subsubsection{Eigenfunctions of $\tilde{K}_{1}$ and $\tilde{K}_{2}$}

Like the operators $\tilde{Q}$ and $\tilde{P}$, which as generators of non-compact groups in general have a continuous spectrum, the self-adjoint operators $\tilde{K}_{1}$ and $\tilde{K}_{2}$ as generators of non-compact groups have a real continuous spectrum. Their "eigenfunctions" may be determined as solutions of differential eqs.:

It follows from Eqs. (452) and (453) that

$$
\tilde{K}_{1}=\frac{1}{2}\left(\tilde{K}_{+}+\tilde{K}_{-}\right)=\cos \vartheta \frac{1}{i} \partial_{\vartheta}+\frac{1}{2} e^{i \vartheta}, \tilde{K}_{2}=\frac{1}{2 i}\left(\tilde{K}_{+}-\tilde{K}_{-}\right)=\sin \vartheta \frac{1}{i} \partial_{\vartheta}+\frac{1}{2 i} e^{i \vartheta} .
$$

It is helpful to observe that $\tilde{K}_{1}$ is obtained from $\tilde{K}_{2}$ by the substituation $\vartheta \rightarrow \vartheta+\pi / 2$. The eigenvalue equation

$$
\tilde{K}_{2} f_{h_{2}}(\vartheta)=h_{2} f_{h_{2}}(\vartheta), h_{2} \in \mathbb{R}
$$

leads to

$$
\left(\partial_{\vartheta} f_{h_{2}}\right) / f_{h_{2}}=\frac{i h_{2}}{\sin \vartheta}-\frac{1}{2}(\cot \vartheta+i) .
$$

As $\sin \vartheta$ and $\tan (\vartheta / 2)$ are positive in the (open) interval $(0, \pi)$ and negative in $(\pi, 2 \pi)$ one has to treat the two intervals slightly differently. For the first interval we get

$$
\begin{aligned}
f_{h_{2}}(\vartheta)= & C_{1} e^{-i \vartheta / 2}(\sin \vartheta)^{-1 / 2}[\tan (\vartheta / 2)]^{i h_{2}} \\
= & \frac{C_{1}}{\sqrt{2}} e^{-i \vartheta / 2}[\sin (\vartheta / 2)]^{i h_{2}-1 / 2}[\cos (\vartheta / 2)]^{-i h_{2}-1 / 2}, \\
& C_{1}=\text { const. }, \vartheta \in(0, \pi) .
\end{aligned}
$$


For the second we get, with $(\sin \vartheta)^{-1 / 2}=e^{i \pi / 2}(|\sin \vartheta|)^{-1 / 2}$ and $\ln \tan (\vartheta / 2)=\ln |\tan (\vartheta / 2)|+$ $i \pi$,

$$
\begin{aligned}
f_{h_{2}}(\vartheta)= & C_{2} e^{-i \pi / 2} e^{-\pi h_{2}} e^{-i \vartheta / 2}(|\sin \vartheta|)^{-1 / 2}[|\tan (\vartheta / 2)|]^{i h_{2}} \\
= & \frac{C_{2}}{\sqrt{2}} e^{-i \pi / 2} e^{-\pi h_{2}} e^{-i \vartheta / 2}[\sin (\vartheta / 2)]^{i h_{2}-1 / 2}[|\cos (\vartheta / 2)|]^{-i h_{2}-1 / 2}, \\
& C_{2}=\text { const. }, \vartheta \in(\pi, 2 \pi) .
\end{aligned}
$$

The three constant factors in the last expression may be combined to $C_{1}=C_{2} e^{-i \pi / 2} e^{-\pi h_{2}}$. For $\vartheta=0, \pi$ the functions (542) become singular, so do the functions (543) for $\vartheta=\pi, 2 \pi$. The constant $C_{1}$ can be determined like in the case of plane waves: Substituting

$$
u(\vartheta)=\ln [\tan (\vartheta / 2)], d u=\frac{d \vartheta}{\sin \vartheta}, u\left(\vartheta \rightarrow 0^{+}\right) \rightarrow-\infty, u\left(\vartheta \rightarrow \pi^{-}\right) \rightarrow+\infty,
$$

into

$$
\frac{1}{2 \pi} \int_{0}^{\pi} d \vartheta f_{h_{2}^{\prime}}^{*}(\vartheta) f_{h_{2}}(\vartheta)=\frac{|C|^{2}}{2 \pi} \int_{0}^{\pi} \frac{d \vartheta}{\sin \vartheta}[\tan (\vartheta / 2)]^{i\left(h_{2}-h_{2}^{\prime}\right)}
$$

yields

$$
\frac{1}{2 \pi} \int_{0}^{\pi} d \vartheta f_{h_{2}^{\prime}}^{*}(\vartheta) f_{h_{2}}(\vartheta)=\frac{|C|^{2}}{2 \pi} \int_{-\infty}^{\infty} d u e^{i u\left(h_{2}-h_{2}^{\prime}\right)}=\left|C_{1}\right|^{2} \delta\left(h_{2}-h_{2}^{\prime}\right) .
$$

The interval $(\pi, 2 \pi)$ gives the same contribution, so that the "normalized" eigenfunctions of $\tilde{K}_{2}$ are

$$
\begin{aligned}
f_{h_{2}}(\vartheta)= & e^{-i \vartheta / 2}(2|\sin \vartheta|)^{-1 / 2}[|\tan (\vartheta / 2)|]^{i h_{2}} \\
= & \frac{1}{2} e^{-i \vartheta / 2}[\sin (\vartheta / 2)]^{i h_{2}-1 / 2}[\cos (\vartheta / 2)]^{-i h_{2}-1 / 2} \\
& \vartheta \in(0, \pi),(\pi, 2 \pi), h_{2} \in \mathbb{R} .
\end{aligned}
$$

Implementing the substitution $\vartheta+\pi / 2$ we get - up to an irrelevant phase factor - the eigenfuctions of $\tilde{K}_{1}$ :

$$
\begin{aligned}
f_{h_{1}}(\vartheta)= & e^{-i \vartheta / 2}(2|\cos \vartheta|)^{-1 / 2}[|\tan (\vartheta / 2+\pi / 4)|]^{i h_{1}}, \\
& \vartheta \in(-\pi / 2, \pi / 2),(\pi / 2,3 \pi / 2), h_{1} \in \mathbb{R}, \\
& \tan (\vartheta / 2+\pi / 4)=(\sin \vartheta+1) / \cos \vartheta
\end{aligned}
$$

For the coefficients $c_{n}$ in the expansion

$$
f_{h_{2}}(\vartheta)=\sum_{n=0}^{\infty} c_{n} e^{i n \vartheta}
$$

one gets [108]

$$
\begin{aligned}
c_{n} & =\frac{1}{2 \pi} \int_{0}^{2 \pi} d \vartheta f_{h_{2}}(\vartheta) e^{-i n \vartheta} \\
& =\frac{1}{2 \pi} \int_{0}^{\pi} d \varphi(\cos \varphi)^{-i h_{2}-1 / 2}(\sin \varphi)^{i h_{2}-1 / 2} e^{-2 i(n+1 / 2) \varphi} \\
& =e^{-i \pi\left(n+1 / 4-i h_{2} / 2\right)} \frac{\Gamma\left(1 / 2+i h_{2}\right)}{n ! \Gamma\left(1 / 2-n+i h_{2}\right)} F\left(1 / 2+i h_{2},-n ; 1 / 2-n+i h_{2} ; z=-1\right) \\
& =(-1)^{n} e^{-i \pi / 4} e^{-h_{2} / 2} \sum_{m=0}^{n}(-1)^{m} \frac{\Gamma\left(1 / 2+i h_{2}+m\right)(-n)_{m}}{\Gamma\left(1 / 2+i h_{2}-n+m\right) m !},
\end{aligned}
$$


where

$$
F(a, b ; c ; z)=\sum_{m=0}^{\infty} \frac{(a)_{m}(b)_{m}}{(c)_{m} m !} z^{m}
$$

is the standard series for the hypergeometric function.

The relation (550) holds for $h_{2}>0$. For $h_{2}<0$ one has to replace $h_{2}$ in Eq. (550) by $\left|h_{2}\right|$. Examples:

$$
c_{0}=e^{-i \pi / 4} e^{-\pi h_{2} / 2},\left|c_{0}\right|^{2}=e^{-\pi h_{2}} ; \quad c_{1}=-2 i e^{-i \pi / 4} h_{2} e^{-\pi h_{2} / 2},\left|c_{1}\right|^{2}=4 h_{2}^{2} e^{-\pi h_{2}} .
$$

\subsubsection{Relationship to the conventional description of the $\mathbf{H O}$ on $L^{2}(\mathbb{R}, d x)$}

The relationship between the quantum mechanical description of the HO in the above Hilbert space $H_{+}^{2}\left(S^{1}, d \vartheta\right)$ and the usual one on $L^{2}(\mathbb{R}, d \xi)$ has been discussed in some detail in chap. 4 of Ref. [13]. I here merely summarize the main steps:

1. The space $H_{+}^{2}\left(S^{1}, d \vartheta\right)$ is mapped unitarily onto the Hardy space $H_{+}^{2}(\mathbb{R}, d \xi)$ of the real line, the elements of which are boundary values $\lim _{\eta \rightarrow 0^{+}} g(z=\xi+i \eta)$ of functions which are holomorphic in the upper half $(\eta>0)$ of the complex plane.

2. The space $L^{2}(\mathbb{R}, d \xi)$ is projected on $H_{+}^{2}(\mathbb{R}, d \xi)$ by the following Fourier tranformations

$$
\begin{aligned}
\hat{g}(p) & =\frac{1}{\sqrt{2 \pi}} \int_{-\infty}^{\infty} d \xi g(\xi) e^{-i \xi p}, \quad g(\xi) \in L^{2}(\mathbb{R}, d \xi) \\
g^{(+)}(\xi) & =\frac{1}{\sqrt{2 \pi}} \int_{0}^{\infty} d p \hat{g}(p) e^{i p \xi}, \quad g^{(+)}(\xi) \in H_{+}^{2}(\mathbb{R}, d \xi) .
\end{aligned}
$$

\subsection{The general case $k>0$}

In case nature "allows" for quantized harmonic oscillators with ground state energies for which $k \neq 1 / 2$, especially $k \in(0,1 / 2)$, then one needs corresponding Hilbert spaces for the description of such systems. I shall briefly mention three examples which may be useful and which are all unitarily equivalent: The Hilbert space of holomorphic functions on the unit circle as described by the Eqs. (410) - (418) in the subsection 6.4.3 above, Hilbert spaces associated with the Hardy space on the circle given by Eqs. (446) - (448) and the Hilbert space $L^{2}([0, \infty), d u)$ on the positive real line with Laguerre's functions as basis.

One can use the Hardy space (410) - (418) itself by using a Holstein-Primakoff variant [27] for the Lie algebra generators

$$
\begin{aligned}
\tilde{K}_{0} & =\frac{1}{i} \partial_{\vartheta}+k \\
\tilde{K}_{+} & =e^{i \vartheta}[(N+2 k)(N+1)]^{1 / 2}, N=\frac{1}{i} \partial_{\vartheta} . \\
\tilde{K}_{-} & =[(N+2 k)(N+1)]^{1 / 2} e^{-i \vartheta} .
\end{aligned}
$$

These operators have the properties (249) - (251) when applied to the basis (524) and one has $\left(f_{2}, \tilde{K}_{+} f_{1}\right)_{+}=\left(\tilde{K}_{-} f_{2}, f_{1}\right)$ for functions (447). For $k=1 / 2$ the operators (555)-(557) reduce to the ones in Eqs. (451) - (453). For $k \neq 1 / 2$ the roots in the expressions (556) and (557) become cumbersome and unpleasent to deal with. They will not be discussed here further. They might, however, be quite useful under certain circumstances. 


\subsubsection{Hilbert space of holomorphic functions on the unit disc}

In subsec. 6.4.3 above I have indicated in connection with Eqs. (417) and (418) that the Hilbert space of holomorphic functions on the unit disc $\mathbb{D}=\{\lambda \in \mathbb{C},|\lambda|<1\}$ with the scalar product (410) can provide irreducible unitary representations of the group $S O^{\uparrow}(1,2)$

and all its covering groups with $k>0$, the self-adjoint generators given by Eq. (450) (see also Appendix B).

The complex numbers $\lambda \in \mathbb{D}$ were introduced in Eq. (311) as eigenvalues of the operator (312). It appears helpful to introduce a new complex variable $\omega \in \mathbb{D}$ (not to be confused with the circular frequency) in order to distinguish the Hilbert space variable in Eqs. (410) - (418) from the eigenvalue $\lambda$. So we have

$$
\begin{aligned}
\left(f_{2}, f_{1}\right)_{k, \omega} & \equiv \int_{\mathbb{D}} d \tilde{\mu}_{k}(\omega) f_{2}^{*}(\omega) f_{1}(\omega), \\
d \tilde{\mu}_{k}(\omega) & =\frac{2 k-1}{\pi}\left(1-|\omega|^{2}\right)^{2 k-2}|\omega| d|\omega| d \theta, \\
\tilde{e}_{k, n}(\omega) & =\sqrt{\frac{(2 k)_{n}}{n !}} \omega^{n},\left(\tilde{e}_{k, n_{2}}, \tilde{e}_{k, n_{1}}\right)_{k, \omega}=\delta_{n_{2} n_{1}}, \\
\left(f_{2}, f_{1}\right)_{k, \omega}=\sum_{n=0}^{\infty} \frac{n !}{(2 k)_{n}} b_{n, 2}^{*} b_{n, 1}, & f_{j}(\omega)=\sum_{n=0}^{\infty} b_{n, j} \omega^{n}, j=1,2,
\end{aligned}
$$

and

$$
\tilde{K}_{0}=\omega \frac{d}{d \omega}+k, \quad \tilde{K}_{+}=\omega\left(2 k+\omega \frac{d}{d \omega}\right), \quad \tilde{K}_{-}=\frac{d}{d \omega},
$$

with the usual properties

$$
\begin{aligned}
\tilde{K}_{0} \tilde{e}_{k, n} & =(n+k) \tilde{e}_{k, n} \\
\tilde{K}_{+} \tilde{e}_{k, n} & =\sqrt{(2 k+n)(n+1)} \tilde{e}_{k, n+1}, \\
\tilde{K}_{-} \tilde{e}_{k, n} & =\sqrt{(2 k+n-1) n} \tilde{e}_{k, n-1} .
\end{aligned}
$$

The associated ladder operators

$$
A=\left(\tilde{K}_{0}+k\right)^{-1 / 2} \tilde{K}_{-}, A^{\dagger}=\tilde{K}_{+}\left(\tilde{K}_{0}+k\right)^{-1 / 2}
$$

have the conventional $k$-independent Fock space properties

$$
A \tilde{e}_{k, n}=\sqrt{n} \tilde{e}_{k, n-1}, \quad A^{\dagger} \tilde{e}_{k, n}=\sqrt{n+1} \tilde{e}_{k, n+1} .
$$

Inserting the number state basis functions (559) into the right-hand sides of the Eqs. (314), (316) and (317) yields the coherent state functions of $\omega$ :

$$
\begin{aligned}
|k, z\rangle(\omega) \equiv f_{k, z}(\omega) & =\frac{e^{z \omega}}{\sqrt{g_{k}\left(|z|^{2}\right)}}, \\
|k, \lambda\rangle(\omega) \equiv f_{k, \lambda}(\omega) & =\frac{\left(1-|\lambda|^{2}\right)^{k}}{(1-\lambda \omega)^{2 k}}, \\
|k, \alpha\rangle(\omega) \equiv f_{k, \alpha}(\omega) & =e^{-|\alpha|^{2} / 2} \sum_{n=0}^{\infty} \frac{\sqrt{(2 k)_{n}}}{n !}(\alpha \omega)^{n} .
\end{aligned}
$$

The general properties of the three types of coherent states as discussed in subsecs. 6.1 and 6.2 are, of course, here valid, too, and will not be repeated. 


\subsubsection{Hilbert spaces related to the Hardy space on the circle}

The scalar product (560) as a series can be implemented on the Hardy space $H_{+}^{2}\left(S^{1}, d \vartheta\right)$ in the following way:

Let us introduce [109] the following positive definite (self-adjoint) operator $A_{k}$ by

$$
A_{k} e_{n}(\vartheta)=\frac{n !}{(2 k)_{n}} e_{n}(\vartheta), \quad e_{n}(\vartheta)=e^{i n \vartheta}, n=0,1, \ldots
$$

Then we can define an additional scalar product for functions

$$
f_{j}(\vartheta)=\sum_{n=0}^{\infty} c_{n, j} e_{n}(\vartheta), j=1,2,
$$

by

$$
\left(f_{2}, f_{1}\right)_{k,+} \equiv\left(f_{2}, A_{k} f_{1}\right)_{+}=\sum_{n=0}^{\infty} \frac{n !}{(2 k)_{n}} c_{n, 2}^{*} c_{n, 1} .
$$

The series here is obviously of the same type as the one in Eq. (560). As

$$
\frac{n !}{(2 k)_{n}}=\left\{\begin{array}{lll}
<1 & \text { for } & k>1 / 2, n>0 \\
=1 & \text { for } & k=1 / 2, \\
>1 & \text { for } & 0<k<1 / 2, n>0
\end{array}\right.
$$

one might suspect that these coefficients affect the convergence properties of the series (572). However, as

$$
\lim _{n \rightarrow \infty}\left(\frac{n !}{(2 k)_{n}}\right)^{1 / n}=1 \text { for } k>0,
$$

the radius of convergence of that series is the same with or without the factor (573) (according to the Cauchy criterium [110])!

Let us denote the (Hardy space associated) Hilbert space with the scalar product (572) by $H_{k,+}^{2}\left(S^{1}, d \vartheta\right)$. An orthonormal basis in this Hilbert space is given by

$$
\hat{e}_{k, n}(\vartheta)=\sqrt{\frac{(2 k)_{n}}{n !}} e_{n}(\vartheta),\left(\hat{e}_{k, n_{2}}, \hat{e}_{k, n_{1}}\right)_{k,+}=\delta_{n_{2} n_{1}} .
$$

From the expressions (561) one can infer (taking the limit $\omega \rightarrow \exp (i \vartheta)$ that

$$
\tilde{K}_{0}=\frac{1}{i} \partial_{\vartheta}+k, \tilde{K}_{+}=e^{i \vartheta}\left(\frac{1}{i} \partial_{\vartheta}+2 k\right), \tilde{K}_{-}=e^{-i \vartheta} \frac{1}{i} \partial_{\vartheta},
$$

with the right properties for the basis (575):

$$
\begin{aligned}
\tilde{K}_{0} \hat{e}_{k, n} & =(n+k) \hat{e}_{k, n}, \\
\tilde{K}_{+} \hat{e}_{k, n} & =\sqrt{(2 k+n)(n+1)} \hat{e}_{k, n+1}, \\
\tilde{K}_{-} \hat{e}_{k, n} & =\sqrt{(2 k+n-1) n} \hat{e}_{k, n-1} .
\end{aligned}
$$

The operators (576) do not have these properties with respect to the basis $e_{n}(\vartheta)$ ! Correspondingly the operators $\tilde{K}_{+}$and $\tilde{K}_{-}$are adjoint to each other only with respect to the scalar 
product (572), not with respect to (448). Their adjointness as to (572) can be verified by taking two series

$$
f_{j}(\vartheta)=\sum_{n=0}^{\infty} a_{n, j} \hat{e}_{k, n}(\vartheta), j=1,2
$$

and showing that $\left(\tilde{K}_{-} f_{2}, f_{1}\right)_{k,+}=\left(f_{2}, \tilde{K}_{+} f_{1}\right)_{k,+}$ !

Note that

$$
\begin{aligned}
\left(e_{n_{2}}, \hat{e}_{k, n_{1}}\right)_{+} & =\left(\hat{e}_{k, n_{1}}, e_{n_{2}}\right)_{+}=\sqrt{\frac{(2 k)_{n_{1}}}{n_{1} !}} \delta_{n_{2} n_{1}} ; \\
\left(e_{n_{2}}, \hat{e}_{k, n_{1}}\right)_{k,+} & =\left(\hat{e}_{k, n_{1}}, e_{n_{2}}\right)_{k,+}=\sqrt{\frac{n_{1} !}{(2 k)_{n_{1}}}} \delta_{n_{2} n_{1}} ; \\
\left(\hat{e}_{k, n_{2}}, \hat{e}_{k, n_{1}}\right)_{+} & =\frac{(2 k)_{n_{1}}}{n_{1} !} \delta_{n_{2} n_{1}}, \quad\left(e_{n_{1}}, e_{n_{2}}\right)_{k,+}=\frac{n_{1} !}{(2 k)_{n_{1}}} \delta_{n_{2} n_{1}} .
\end{aligned}
$$

The Fock space ladder operators $A$ and $A^{\dagger}$ associated with the Lie algebra generators (576) are given in the same way as in Eq. (565).

\section{Coherent state wave functions}

Analogously to the relations (567) - (569) we obtain on $H_{k,+}^{2}$ the following coherent state wave functions by using the basis (575):

$$
\begin{aligned}
|k, z\rangle(\vartheta) \equiv f_{k, z}(\vartheta) & =\frac{e^{z e^{i \vartheta}}}{\sqrt{g_{k}\left(|z|^{2}\right)}} \\
|k, \lambda\rangle(\vartheta) \equiv f_{k, \lambda}(\vartheta) & =\frac{\left(1-|\lambda|^{2}\right)}{\left(1-\lambda e^{i \vartheta}\right)^{2 k}} \\
|k, \alpha\rangle(\vartheta) \equiv f_{k, \alpha}(\vartheta) & =e^{-|\alpha|^{2} / 2} \sum_{n=0}^{\infty} \frac{\sqrt{(2 k)_{n}}}{n !}\left(\alpha e^{i \vartheta}\right)^{n}
\end{aligned}
$$

The reproducing kernel on $H_{k,+}^{2}$ is given by

$$
\hat{A}_{k}\left(\vartheta_{2}-\vartheta_{1}\right)=\sum_{n=0}^{\infty} \hat{e}_{k, n}^{*}\left(\vartheta_{2}\right) \hat{e}_{k, n}\left(\vartheta_{1}\right)=\left[1-e^{i\left(\vartheta_{1}-\vartheta_{2}\right)}\right]^{-2 k}=\hat{A}_{k}^{*}\left(\vartheta_{1}-\vartheta_{2}\right)
$$

According to the relations $(\underline{581})$ - (583) it has the properties

$$
\begin{aligned}
\left(\hat{A}_{k}(1,2), \hat{e}_{k, m}(2)\right)_{k,+} & =\hat{e}_{k, m}\left(\vartheta_{1}\right) \\
\left(\hat{A}_{k}(1,2), \hat{e}_{k, m}(2)\right)_{+} & =\frac{(2 k)_{m}}{m !} \hat{e}_{k, m}\left(\vartheta_{1}\right), \\
\left(\hat{A}_{k}(1,2), e_{m}(2)\right)_{k,+} & =\sqrt{\frac{m !}{(2 k)_{m}}} \hat{e}_{k, m}\left(\vartheta_{1}\right)=e_{m}\left(\vartheta_{1}\right), \\
\left(\hat{A}_{k}(1,2), e_{m}(2)\right)_{+} & =\sqrt{\frac{(2 k)_{m}}{m !}} \hat{e}_{k, m}\left(\vartheta_{1}\right)=\frac{(2 k)_{m}}{m !} e_{m}\left(\vartheta_{1}\right) .
\end{aligned}
$$

The numbers 1 and 2 mean the variables $\vartheta_{1}$ and $\vartheta_{2}$, the latter being integration variable. 


\section{A unitary transformation}

In the above discussion the $k$-dependence of the representation is contained in the operators (576), not in the basis $e_{n}(\vartheta)$ of $H_{+}^{2}$ we started from. Like in subsection 7.1.4 one can shift the $k$-dependence partially from the operators to the basis by a unitary transformation:

$$
e_{n}(\vartheta)=e^{i n \vartheta} \rightarrow e_{k, n}(\vartheta)=e^{i(n+k) \vartheta},
$$

the generators (576) now taking the form

$$
\tilde{K}_{0}=\frac{1}{i} \partial_{\vartheta}, \tilde{K}_{+}=e^{i \vartheta}\left(\frac{1}{i} \partial_{\vartheta}+k\right), \tilde{K}_{-}=e^{-i \vartheta}\left(\frac{1}{i} \partial_{\vartheta}-k\right) .
$$

The operators (593) act in a Hilbert space $H_{k,+}^{2}$, now with the orthonormal basis

$$
\hat{e}_{k, n}(\vartheta)=\sqrt{\frac{(2 k)_{n}}{n !}} e_{k, n}(\vartheta)
$$

The basis functions (592) are no longer periodic but quasi-periodic:

$$
e_{k, n}(\vartheta+2 \pi)=e^{2 i k \pi} e_{k, n}(\vartheta) .
$$

These functions are special Bloch-type wave functions on the circle [25].

\subsubsection{Hilbert space on the positive real line}

There exists a unitary mapping [111] from the Hilbert space of holomorphic functions on the unit disc as characterized by the Eqs. (558) and (559) to the Hilbert space $L^{2}\left(\mathbb{R}_{+}, d u\right)$, where $\mathbb{R}_{+}=[0, \infty)$, i.e. we have the scalar product

$$
\left(f_{2}, f_{1}\right)=\int_{0}^{\infty} d u f_{2}^{*}(u) f_{1}(u)
$$

for functions $f(u)$ on $\mathbb{R}_{+}$. The standard orthonormal basis on this space are Laguerre's functions [112], slightly adapted for our purposes,

$$
\breve{e}_{k, n}(u)=\sqrt{\frac{n !}{\Gamma(2 k)(2 k)_{n}}} u^{k-1 / 2} e^{-u / 2} L_{n}^{2 k-1}(u), k>0,
$$

where the functions $L_{n}^{\alpha}(u)$ are Laguerre's polynomials

$$
L_{n}^{\alpha}(u)=\sum_{m=0}^{m=n}\left(\begin{array}{c}
n+\alpha \\
n-m
\end{array}\right) \frac{(-u)^{m}}{m !}, \quad L_{n}^{\alpha}(0)=\frac{(\alpha+1)_{n}}{n !} .
$$

These have the generating function [112]

$$
\sum_{n=0}^{\infty} L_{n}^{2 k-1}(u) \omega^{n}=(1-\omega)^{-2 k} e^{-u \omega /(1-\omega)}, \omega \in \mathbb{D} .
$$

This implies that

$$
B_{k}(\omega, u)=\sum_{n=0}^{\infty} \tilde{e}_{k, n}(\omega) \breve{e}_{k, n}(u)=\frac{1}{\sqrt{\Gamma(2 k)}}(1-\omega)^{-2 k} u^{k-1 / 2} e^{-(u / 2)(1+\omega) /(1-\omega)},
$$


where $\tilde{e}_{k, n}(\omega)$ denotes the basis (559). The function $B_{k}(\omega, u)$ is by construction the kernel of a unitary transformation from the basis $\breve{e}_{k, n}$ to the basis $\tilde{e}_{k, n}, B_{k}^{*}(\omega, u)$ being the kernel for the inverse transformation:

$$
\int_{0}^{\infty} d u B_{k}(\omega, u) \breve{e}_{k, n}=\tilde{e}_{k, n}(\omega), \quad \int_{\mathbb{D}} d \tilde{\mu}_{k}(\omega) B_{k}^{*}(\omega, u) \tilde{e}_{k, n}(\omega)=\breve{e}_{k, n}(u) .
$$

One can show [113] that the operators $\tilde{K}_{0}, \tilde{K}_{1}$ and $\tilde{K}_{2}$ now have the form

$$
\begin{aligned}
\tilde{K}_{0} & =-u \frac{d^{2}}{d u^{2}}-\frac{d}{d u}+\frac{(2 k-1)^{2}}{4 u}+\frac{u}{4}, \quad \tilde{K}_{0} \breve{e}_{k, n}(u)=(n+k) \breve{e}_{k, n}(u), \\
\tilde{K}_{1} & =-u \frac{d^{2}}{d u^{2}}-\frac{d}{d u}+\frac{(2 k-1)^{2}}{4 u}-\frac{u}{4} \\
\tilde{K}_{2} & =\frac{1}{i}\left(u \frac{d}{d u}+1 / 2\right)
\end{aligned}
$$

As

$$
\tilde{K}_{0}-\tilde{K}_{1}=\frac{u}{2}
$$

the integration variable $u$ may be associated with the classical quantity

$$
\tilde{h}_{0}-\tilde{h}_{1}=\tilde{I}(1-\cos \varphi)=2 \tilde{I} \sin ^{2}(\varphi / 2)
$$

that is to say we have the correspondence

$$
u \leftrightarrow 4 \tilde{I} \sin ^{2}(\varphi / 2) \geq 0
$$

Inserting $\breve{e}_{k, n}(u)$ for the general number state $|k, n\rangle$ into the series (314), (316) and (317) yields the following coherent state wave functions

$$
\begin{aligned}
|k, z\rangle(u) \equiv f_{z}(u) & =\frac{u^{k-1 / 2} e^{-u / 2}}{\sqrt{g_{k}\left(|z|^{2}\right)}} \sum_{n=0}^{\infty} \frac{z^{n}}{\Gamma(2 k+n)} L_{n}^{2 k-1}(u) \\
& =\frac{u^{k-1 / 2} e^{(z-u / 2)}}{\sqrt{g_{k}\left(|z|^{2}\right)}}(u z)^{-k+1 / 2} J_{2 k-1}(2 \sqrt{u z}) \\
& =\frac{u^{k-1 / 2} e^{(z-u / 2)}}{\sqrt{g_{k}\left(|z|^{2}\right)}} \sum_{n=0}^{\infty} \frac{(-u z)^{n}}{n ! \Gamma(2 k+n)} \\
& =u^{k-1 / 2} e^{(z-u / 2)} \frac{g_{k}(-u z)}{\sqrt{g_{k}\left(|z|^{2}\right)}}
\end{aligned}
$$

where the relations [112]

$$
\begin{aligned}
\sum_{n=0}^{\infty} \frac{z^{n}}{\Gamma(2 k+n)} L_{n}^{2 k-1}(u) & =e^{z}(u z)^{-k+1 / 2} J_{2 k-1}(2 \sqrt{u z}) \\
J_{\nu}(\zeta) & =(\zeta / 2)^{\nu} \sum_{n=0}^{\infty} \frac{(-1)^{n}}{n ! \Gamma(\nu+n+1)}(\zeta / 2)^{2 n}
\end{aligned}
$$

and (315) have been used. 
The relation (599) implies

$$
|k, \lambda\rangle(u) \equiv f_{\lambda}(u)=\frac{\left(1-|\lambda|^{2}\right)^{k}(1-\lambda)^{-2 k}}{\sqrt{\Gamma(2 k)}} u^{k-1 / 2} e^{-(u / 2)(1+\lambda) /(1-\lambda)} .
$$

Finally

$$
|k, \alpha\rangle(u) \equiv f_{\alpha}(u)=\frac{1}{\sqrt{\Gamma(2 k)}} e^{-\left(|\alpha|^{2}+u\right) / 2} u^{k-1 / 2} \sum_{n=0}^{\infty} \frac{\alpha^{n}}{\sqrt{(2 k)_{n}}} L_{n}^{2 k-1}(u) .
$$

Let us have a brief look at the behaviour of the probability densities

$$
p_{k, n}(u)=\left|\breve{e}_{k, n}(u)\right|^{2}=\frac{n !}{\Gamma(2 k)(2 k)_{n}} u^{2 k-1} e^{-u}\left|L_{n}^{2 k-1}(u)\right|^{2}
$$

for small $u$ as a function of $k$. Because of the second of the relations (598) we have

$$
p_{k, n}\left(u \rightarrow 0^{+}\right) \approx \frac{1}{\Gamma(2 k)} u^{2 k-1} .
$$

Thus $p_{k, n}(u)$ vanish in the limit $u \rightarrow 0$ for $k>1 / 2$, has the finite value 1 for $k=1 / 2$ and diverges for $0<k<1 / 2$ (but is still integrable). Notice that the behaviour (614) is independent of $n$.

As $\Gamma(2 k)$ behaves like $1 /(2 k)$ near $k=0$ we have

$$
p_{k, n}\left(u \rightarrow 0^{+} ; k \rightarrow 0^{+}\right) \approx 2 k u^{2 k-1} .
$$

The ground state probability density is

$$
p_{k, n=0}(u)=\frac{1}{\Gamma(2 k)} u^{2 k-1} e^{-u} .
$$

For $k>1 / 2$ it has a maximum at $u=u_{0}=2 k-1$.

The eigenfunctions $f_{h_{2}}(u)$ of the operator $\tilde{K}_{2}$ in Eq. (604) can easily be found as

$$
f_{h_{2}}(u)=\frac{1}{\sqrt{2 \pi}} u^{i h_{2}-1 / 2}, \quad \int_{0}^{\infty} d u f_{h_{2}^{\prime}}(u) f_{h_{2}}(u)=\delta\left(h_{2}^{\prime}-h_{2}\right) .
$$

The last relation can be verified by the substitution $u=e^{v}, d u=u d v$. The eigenfunctions, which are independent of $k$, can be used for Mellin transformations [114]

$$
\hat{g}(s)=\int_{0}^{\infty} d u g(u) u^{s-1}, \quad s=i h_{2}+1 / 2,
$$

with the inversion

$$
g(u)=\frac{1}{2 \pi i} \int_{1 / 2-i \infty}^{1 / 2+i \infty} d s u^{-s} \hat{g}(s) .
$$

The substitution $u=e^{v}$ shows the close relationship of the Mellin transform to the Fourier transform.

The eigenfunctions of $\tilde{K}_{1}$ are more complicated [115]:

$$
f_{h_{1}}(u)=C u^{k-1 / 2} e^{-i u / 2} \Phi\left(k-i h_{1} ; 2 k ; i u\right), C=\text { const. },
$$

where $\Phi(a ; c ; z)$ is the confluent hypergeometric series

$$
\Phi(a ; c ; z)=\sum_{n=0}^{\infty} \frac{(a)_{n}}{(c)_{n} n !} z^{n} .
$$




\section{On the ground state of the quantized free electro- magnetic field in a cavity}

\subsection{The electromagnetic field in a cavity as a set of harmonic os- cillators}

The standing free electromagnetic waves in a cavity can be interpreted as a denumerable set of harmonic oscillators each of them having the ground state energy (5), the sum of which is infinite! This "nuisance" led to the concept of "normal-ordering", which just means to ignore the infinite ground state energies. On the other hand, subtracting two such infinities leads to the Casimir effect $[8,15,16]$, a quantum ("vacuum") force between two ideally conducting plates, now experimentally verified [116]. The effect can, however, also be derived without refering to vacuum energies and their fluctuations, by subtracting appropriate Green's functions associated with certain boundary conditions [117].

The issue of quantum vacuum energies assumes "cosmic" dimensions in the context of the cosmological constant in Einstein's theory of gravity. The usual estimates for that constant are essentially based on the value (5). Those estimates turn out to be up to more than 100 orders of magnitudes larger than the experimentally determined value, the estimate depending on the cutoff chosen. This discrepancy obviously constitutes the most urgent and provocative challenge as to the quantitative powers of physical theories. The issue has become very acute recently by the observation of an appreciable "dark energy" in the universe (about $75 \%$ of all matter), very likely related to the gravitational cosmological constant and the associated "vacuum energies" [17-24].

It is obvious that the much richer spectrum of possible ground states for the HO Hamiltonian (18) can shed new light on the subject. I here shall only point out the crucial part of the issue without going into further details.

I first recall the main elements as to the formulation of standing waves in a cubic cavity with side lengths $L$ in terms of harmonic oscillators [118], with the slight generalization (compared to most textbooks) to allow for relative dielectric constants $\epsilon$ and relative magnetic permeabilities $\mu$ different from the vacuum values $\epsilon=1, \mu=1$ :

In the Coulomb gauge Maxwell's equations without sources are given by

$$
\frac{1}{v^{2}} \partial_{t}^{2} \vec{A}-\Delta \vec{A}=0 ; \operatorname{div} \vec{A}=0, \quad \vec{E}=-\partial_{t} \vec{A}, \quad \vec{B}=\operatorname{curl} \vec{A}, \quad v=c / n, \quad n=+\sqrt{\epsilon \mu}
$$

Postulating periodic boundary conditions for the vector potential leads to the solution type

$$
\vec{A}(t, \vec{x})=\frac{1}{\sqrt{\epsilon_{0} L^{3}}} \sum_{\vec{m} \in \mathbb{Z}^{3}} \vec{A}_{\vec{l}}(t) e^{i \vec{l} \cdot \vec{x}}+\vec{A}_{\vec{l}}^{*}(t) e^{-i \vec{l} \cdot \vec{x}},
$$

where

$$
\vec{l}=\frac{2 \pi}{L} \vec{m}, \vec{m}=\left(m_{1}, m_{2}, m_{3}\right), m_{j} \in \mathbb{Z}, j=1,2,3 ; \vec{l} \cdot \vec{A}_{\vec{l}}(t)=0 .
$$

The time-dependent factors $\vec{A}_{\vec{l}}(t)$ obey the HO equations

$$
\partial_{t}^{2} \vec{A}_{\vec{l}}(t)+\omega^{2}(\vec{l}) \vec{A}_{\vec{l}}(t)=0, \omega^{2}(\vec{l})=v^{2} \vec{l}^{2}=\frac{c^{2}}{n^{2}} \vec{l}^{2}, \omega(\vec{l}) \geq 0
$$


with the solutions

$$
\vec{A}_{\vec{l}}(t)=\sum_{\lambda=1}^{2} \tilde{c}_{\vec{l} \lambda}(t) \vec{\epsilon}_{\vec{l} \lambda}+\tilde{c}_{\vec{l} \lambda}^{*}(t) \vec{\epsilon}_{\vec{l} \lambda}, \quad \tilde{c}_{\vec{l} \lambda}(t)=c_{\overrightarrow{l \lambda}} e^{-i \omega(\vec{l}) t}
$$

where the $\vec{\epsilon}_{\vec{l} \lambda}$ are two polarization vectors.

Inserting the associated electric and magnetic fields into the integral for the electromagnetic field energy in the cavity,

$$
E_{\text {em }}(\text { cavity })=\frac{1}{2} \int_{\text {cavity }} d^{3} x\left[\epsilon \epsilon_{0} \vec{E}^{2}(t, \vec{x})+\frac{1}{\mu \mu_{0}} \vec{B}^{2}(t, \vec{x})\right], \quad \epsilon_{0} \mu_{0}=1 / c^{2},
$$

and observing that for plane wave solutions [119]

$$
\vec{B}^{2}=\epsilon \epsilon_{0} \mu \mu_{0} \vec{E}^{2}=\frac{n^{2}}{c^{2}} \vec{E}^{2},
$$

yields

$$
E_{\text {em }}(\text { cavity })=2 \epsilon \sum_{\vec{m}} \sum_{\lambda}[\omega(\vec{l})]^{2}\left|\tilde{c}_{\vec{l} \lambda}\right|^{2} .
$$

Notice that $\left|\tilde{c}_{\vec{l}, \lambda}(t)\right|^{2}=\left|c_{\vec{l} \lambda}\right|^{2}$. Defining

$$
\tilde{c}_{\vec{l} \lambda}=\frac{1}{2}\left[q_{\vec{l} \lambda}+\frac{i}{\omega(\vec{l})} p_{\vec{l} \lambda}\right], \quad p_{\vec{l} \lambda}=\dot{q}_{\vec{l} \lambda}
$$

the expression (629) finally becomes

$$
E_{e m}(\text { cavity })=H_{e m}(q, p)=\frac{\epsilon}{2} \sum_{\vec{m}} \sum_{\lambda}\left[p_{\vec{l} \lambda}^{2}+\omega^{2}(\vec{l}) q_{\vec{l} \lambda}^{2}\right],
$$

where the individual terms

$$
H_{\vec{l} \lambda}(q, p)=\frac{1}{2}\left[p_{\vec{l} \lambda}^{2}+\omega^{2}(\vec{l}) q_{\vec{l} \lambda}^{2}\right]
$$

are independent of time!

As can be seen from Maxwell's eqs. (622), the electric field provides the canonical momenta, the magnetic field (via its vector potential) the canonical coordinates [120].

The standard quantization procedure is now obvious: The classical quantities $q_{\vec{l} \lambda}$ and $p_{\vec{l} \lambda}$ are promoted to operators $Q_{\vec{l} \lambda}$ and $P_{\vec{l} \lambda}$, having the commutation relations

$$
\left[Q_{\vec{l} \lambda}, P_{\overrightarrow{l^{\prime}} \lambda^{\prime}}\right]=i \hbar \delta_{\vec{l} \vec{l}^{\prime}} \delta_{\lambda \lambda^{\prime}} .
$$

A very minor point may be worth mentioning here: The right-hand side of the energy (631) does not contain a mass term. As the dimension of the energy is given, $\left[L^{2} T^{-2} M\right]$, the quantities $q_{\overrightarrow{l \lambda}}$ and $p_{\vec{l} \lambda}$ here have dimensions $\left[M^{1 / 2} L\right]$ and $\left[M^{1 / 2} L T^{-1}\right]$, respectively. But their product still has the dimension of an action, $\left[M L^{2} T^{-1}\right]$ !

We now come to the point of departure: Assuming (for a moment) $\epsilon=1, \mu=1$ and introducing angle and action variable for each mode,

$$
q_{\vec{l} \lambda}=\sqrt{2 I_{\vec{l} \lambda} / \omega(\vec{l})} \cos \varphi_{\vec{l} \lambda}, \quad p_{\vec{l} \lambda}=-\sqrt{2 \omega(\vec{l}) I_{\vec{l} \lambda}} \sin \varphi_{\vec{l} \lambda},
$$


yields

$$
H_{\vec{l} \lambda}(\varphi, I)=\omega(\vec{l}) I_{\vec{l} \lambda}, \quad H_{e m}(\varphi, I)=\sum_{\vec{m}} \sum_{\lambda} H_{\vec{l} \lambda} .
$$

Quantization proceeds now as discussed above for the angle-action model of the HO: Each of the $H_{\vec{l} \lambda}$ is replaced by an operator

$$
H_{\vec{l} \lambda}(\vec{K})=\hbar \omega(\vec{l}) \tilde{K}_{0}(\vec{l}, \lambda), \quad H_{e m}(\vec{K})=\sum_{\vec{m}} \sum_{\lambda} \oplus H_{\vec{l} \lambda}(\vec{K}) .
$$

Each $\tilde{K}_{0}(\vec{l}, \lambda)$ acts irreducibly in a Hilbert space that carries a unitary representation with Bargmann index $k$, together with the operators $\tilde{K}_{1}(\vec{l}, \lambda)$ and $\tilde{K}_{2}(\vec{l}, \lambda)$ or the ladder operators $\tilde{K}_{+}(\vec{l}, \lambda)$ and $\tilde{K}_{-}(\vec{l}, \lambda)$. Because of the required bosonic exchange symmetries I here assume the same Bargmann index $k$ for all representations. I here do not enter the important subject of constructing and analyzing the quantized free or even interacting electromagnetic fields themselves in terms of the operators $\tilde{K}_{j}(\vec{l}, \lambda)$ etc. The usual $k$-independent annihilation and creation operators associated with the fields themselves are given by

$$
\left.A_{\vec{l}, \lambda}=\left[\tilde{K}_{0}(\vec{l}, \lambda)+k\right]^{-1 / 2} \tilde{K}_{-}(\vec{l}, \lambda)\right), \quad A_{\vec{l}, \lambda}^{\dagger}=\tilde{K}_{+}(\vec{l}, \lambda)\left[\tilde{K}_{0}(\vec{l}, \lambda)+k\right]^{-1 / 2} .
$$

\subsection{The cosmological constant problem}

Presently I am merely interested in the ground state expectation value

$$
\left\langle k, 0_{\otimes}\left|H_{e m}(\vec{K})\right| k, 0_{\otimes}\right\rangle=\sum_{\vec{m}} \sum_{\lambda}\left\langle k, 0\left|H_{\vec{l} \lambda}(\vec{K})\right| k, 0\right\rangle=2 k \hbar \sum_{\vec{m}} \omega(\vec{l}) .
$$

The usual replacement

$$
\sum_{\vec{m}, \lambda} \rightarrow \frac{L^{3}}{\pi^{2} c^{3}} \int_{\omega \geq 0} d \omega \omega^{2}
$$

leads to a strongly divergent ground state energy density

$$
\frac{k \hbar}{\pi^{2} c^{3}} \int_{\omega \geq 0} d \omega \omega^{3} .
$$

Cutting the infinite integral off at $\omega=\hat{\omega}$ yields the "vacuum" energy density

$$
u_{e m, 0}(\hat{\omega}, k)=\frac{k \hbar}{4 \pi^{2} c^{3}} \hat{\omega}^{4} .
$$

Defining the effective length

$$
\ell=\frac{2 \pi c}{\hat{\omega}}
$$

finally gives

$$
u_{e m, 0}(\ell, k)=\frac{4 \pi^{2} k}{\ell^{4}} \hbar c \approx \frac{4 \pi^{2} k}{\ell^{4}} \cdot\left(2 \cdot 10^{-11} \mathrm{MeV} \mathrm{cm}\right) .
$$

We know from sec. 5 that the index $k$ may become arbitrarily small $>0$, perhaps in the course of time - So the $k$ in the expression (643) may become so small - for a given value of 
the interaction length $\ell$ - that the value of $u_{e m, 0}(\ell, k)$ comes near the order of magnitude of the observed dark energy density [121]

$$
c^{2} \rho_{\Lambda} \approx 4 \mathrm{keV} \mathrm{cm}^{-3} .
$$

Such a welcome adjustment of $k$ is, of course, here not proven at all, and one would like to have more sophisticated arguments in the present framework for the desired appropriate value of the index $k$ in order to get a "reasonable" estimate for the cosmological constant. Nevertheless, the mere existence of that index, originating from the non-trivial topology of the $(\varphi, I)$-phase space of the $\mathrm{HO}$ and its related quantizing group $S O^{\uparrow}(1,2)$ (including its infinitely many covering groups), may be an important key for the solution of the cosmological constant problem!

I list a few of the many problems I leave open here:

- The role of the index $k$ has to be examined for other matter fields, especially fermions and non-abelian gauge fields and associated interactions, particularly for those with spontaneous symmetry breaking!

- The compatibility with (local) Poincaré covariance and its concept of causality has to be analyzed.

- Most of the prevailing discussions of the Casimir effect - with their by now quite sophisticated subtractions of two infinities - (see the literature quoted above) and especially their experimental confirmations appear to contradict the introduction of an index $k$ different from $1 / 2$. There are different answers to such an objection:

First, it is evident from the discussions above, that the ground state of the HO Hamiltonian $H(Q, P)$ is necessarily tight to $k=1 / 2$. In order to have $k \neq 1 / 2$ the basic quantum observables have to be the $\tilde{K}_{j}$. An analysis of the Casimir effect in terms of these new variables has not yet been done.

Second, there have been alternative proposals for deriving the Casimir effect (force) instead of subtracting infinite vacuum energies [117]!

- As the number $k$ is a (dimensionless) measure for some energy, it may become timedependent, i.e. dynamical, on a cosmic scale and might lead to a time-dependent cosmological constant. The index $k$ may also become a function of the frequency $\omega$ or (and) of space coordinates, like the dielectic constant $\epsilon$ from above.

\subsection{Birefringence and dichroism of the vacuum}

Comparing the expressions (631) and (638) suggests to preliminarily interpret the index $k$ here as a kind of "anomalous" dielectric constant (or the square of an "anomalous" index of refraction, cf. Eq. (622) ) of the vacuum. This interpretation leads (tentatively) to the following possible quantum optical application:

Lets assume we have in vacuum initially just two photon modes of the same frequency $\omega$, the same initial wave number $\vec{l}$, but orthogonal linear polarizations. Both should initially belong to the same index $k$. If one lets these photons pass through strong electric or (and) static magnetic fields $\vec{E}_{0}, \vec{B}_{0}$, these "perturbations" add constant terms proportional to $\epsilon_{0} \vec{E}_{0}^{2}$ or (and) $\vec{B}_{0}^{2} / \mu_{0}$ to the free Hamiltonians $H_{\vec{l} \lambda}(\vec{K}), \lambda=1,2$, (see also the discussion around 
Eq. (495) ). The energy of the static fields may change the index $k$ of at least one of the fields by a small amount $\delta k$ which could lead to the following possible effects:

- Compared to the photon the vacuum energy of which is "lifted" by an amount $\delta k>0$ the other photon with the orthogonal polarization "lost" energy, leading to an effective "dichroism"!

- If the energetically lifted photon returns to its original index $k$ after passing the external fields, then we have an effective "birefringence"!

As to the conventional optical phenomena of this type in materials (electro-optical "Kerreffect" or magneto-optical "Cotton-Mouton-" and "Voigt-" effects and related dichroisms etc.) see Refs. [122].

The effects mentioned should be proportional to the square of the external electric or (and) magnetic fields.

Possibly the recent PVLAS experiment $[31,123]$ with its observation of vacuum dichroism induced by an external magnetic field can be understood in this framework!

\section{4 "Dark" normal matter?}

Let me dare to add a very speculative remark: As the quantum spectra (20) and (21) of the two HO classical models (3) can be different, the index $k>0$ possibly being very small. So (radiation) energy may get "stuck" in the interval $1 / 2>k>0$ or even at higher excited levels which perhaps can decay by higher order electromagnetic transitions only. In such a speculation dark matter would be just "normal" matter prevented from radiating normally (e.g., the abundance of diatomic molecular hydrogen [124] provides an abundance of effective HOs). This could "explain" why visible and dark matter are of the same order of magnitude! In such a speculative scenario dark matter could have been formed only after the formation of atoms and molecules. All this has, of course, to be evaluated much more critically.

\section{Charged particles in external electric and magnetic fields}

\subsection{Charged harmonic oscillator in an external electric field}

If one puts a harmonically vibrating particle of mass $M$ and charge $Z e_{0}, Z \in \mathbb{Z}$, in an external electric field $E_{0}$ in $q$-direction then the potential term

$$
-Z e_{0} E_{0} q
$$

has to be added to the Hamiltonian of the HO:

$$
H=\frac{1}{2 M} p^{2}+\frac{M}{2} \omega^{2} q^{2}-Z e_{0} E_{0} q=\frac{1}{2 M} p^{2}+\frac{M}{2} \omega^{2}\left(q-\frac{Z e_{0} E_{0}}{\omega^{2} M}\right)^{2}-\frac{Z^{2} e_{0}^{2} E_{0}^{2}}{2 \omega^{2} M} .
$$

Defining

$$
\xi=q-\frac{Z e_{0} E_{0}}{\omega^{2} M}
$$


we again have an effective $\mathrm{HO}$ with coordinate $\xi$ and the ground state energy shifted by the amount

$$
V_{0}=-\frac{Z^{2} e_{0}^{2} E_{0}^{2}}{2 \omega^{2} M} \leq 0
$$

Replacing $q$ in Eq. (2) by $\xi$ yields

$$
H(\xi, p)=\frac{1}{2 M} p^{2}+\frac{M}{2} \omega^{2} \xi^{2}+V_{0}=\omega I+V_{0}
$$

The fine structure constant

$$
\alpha=\frac{e_{0}^{2}}{4 \pi \epsilon_{0} c \hbar} \approx 7.3 \cdot 10^{-3}
$$

allows $V_{0}$ to be rewritten as

$$
-V_{0}=\hbar \omega \frac{2 \pi \alpha Z^{2} \epsilon_{0} E_{0}^{2}}{(\omega / c)^{3} M c^{2}}=\hbar \omega \frac{\alpha Z^{2}}{4 \pi^{2}} \frac{\epsilon_{0} E_{0}^{2} \lambda^{3}}{M c^{2}}, \quad \lambda=\frac{2 \pi c}{\omega}=\frac{c}{\nu} .
$$

Comparing with Eq. (19) suggest to introduce an effective Bargmann index

$$
k \rightarrow k_{e f f}=k-\delta, \quad \delta=\frac{\alpha Z^{2}}{4 \pi^{2}} \frac{\epsilon_{0} E_{0}^{2} \lambda^{3}}{M c^{2}} .
$$

In order to get an impression of the order of magnitude for $\delta$ in experiments consider an ion of rest energy $M c^{2} \approx 100 \mathrm{GeV} \approx 10^{-8} \mathrm{~J}$ and charge $e_{0}$ in a 1-dimensional harmonic Paul trap [36]. With $E_{0} \approx 10^{3} \mathrm{~V} / \mathrm{m}$ along the longitudinal direction, $\nu \approx 10^{8} \mathrm{~Hz}$ one gets approximately the value $\delta \approx 10$, which makes $k_{\text {eff }}$ negative! One further has to reduce the energy $\epsilon_{0} E_{0}^{2} \lambda^{3}$ compared to $M c^{2}$ in order to have $k_{\text {eff }}$ positive.

\subsection{Charged particle in an external magnetic field}

It is well-known that the 3 -dimensional motion of a particle with charge $q$ in a homogeneous magnetic field $\vec{B}=\operatorname{curl} \vec{A}$ can be associated with an effective harmonic oscillator for the motion transversal to the magnetic field [125]: The Hamilton function is given by (here $m$ obviously means the mass, as opposed to previous Secs.)

$$
H=\frac{1}{2 m} \vec{\pi}^{2}, \quad \vec{\pi}=m \dot{\vec{x}}=\vec{p}-q \vec{A}(\vec{x}),
$$

with the basic Poisson brackets

$$
\left\{x_{j}, p_{k}\right\}=\delta_{j k}, j, k=1,2,3 \text {. }
$$

The Eqs. of motion are

$$
\dot{x}_{j}=\left\{x_{j}, H\right\}=\frac{1}{m}\left(p_{j}-q A_{j}\right), \quad \dot{p}_{j}=\left\{p_{j}, H\right\}=q \sum_{k=1}^{3} \dot{x}_{k} \partial_{j} A_{k} .
$$

It follows from the Poisson brackets (654) that

$$
\left\{\pi_{j}, \pi_{k}\right\}=q\left(\partial_{j} A_{k}-\partial_{k} A_{j}\right)=q B_{l}, \quad(j, k, l)=\operatorname{cycl} .(1,2,3) .
$$


For $\vec{B}=(0,0, B)$ we have

$$
\left\{\pi_{1}, \pi_{2}\right\}=q B, \quad\left\{\pi_{1}, \pi_{3}\right\}=0, \quad\left\{\pi_{2}, \pi_{3}\right\}=0 .
$$

The last relations imply

$$
\dot{\pi}_{3}=\left\{\pi_{3}, H\right\}=0
$$

i.e. $\pi_{3}$ is a constant of motion.

Of special interest here is the remaining "transversal" Hamilton function

$$
H_{\perp}=\frac{1}{2 m}\left(\pi_{1}^{2}+\pi_{2}^{2}\right) \text {. }
$$

Defining

$$
\omega=q B / m, \quad \pi_{1}=m \omega \xi, \quad \pi_{2}=\pi_{\xi},
$$

and assuming $q B>0$ we get

$$
H_{\perp}=\frac{1}{2 m} \pi_{\xi}^{2}+\frac{1}{2} m \omega^{2} \xi^{2}, \quad\left\{\xi, \pi_{\xi}\right\}=1 .
$$

This is an effective HO Hamilton function for the transversal motion of a particle with charge $q$ in a magnetic field $\vec{B}=(0,0, B)$. As the "canonical coordinate" $\xi$ actually is proportinal to a time derivative of the original coordinates, one needs another integration. This is provided by the quantities

$$
b_{1}=x_{1}+\frac{1}{m \omega} \pi_{\xi}, \quad b_{2}=x_{2}-\xi=x_{2}-\frac{1}{m \omega} \pi_{1} ; \quad\left(x_{1}-b_{1}\right)^{2}+\left(x_{2}-b_{2}\right)^{2}=\frac{2}{m \omega^{2}} H_{\perp},
$$

which obey

$$
\left\{b_{j}, \pi_{k}\right\}=0, j, k=1,2 ; \quad\left\{b_{2}, b_{1}\right\}=\frac{1}{m \omega},
$$

implying

$$
\left\{b_{j}, H_{\perp}\right\}=0, j=1,2,
$$

i.e. the $b_{j}$ are constants of motion. According to their definition they are the coordinates of the center of the circle on which the particle moves in the tansversal (1,2)-plane.

If $q B<0$ one just has to interchange the roles of $\pi_{1}$ and $\pi_{2}$ in the relations (659) and (660) and defines $\omega=|q B|$.

For the HO Hamilton function (661) one can introduce angle and action variables as usual: With $\omega>0$ and defining

$$
\xi=\sqrt{\frac{2 I}{m \omega}} \cos \varphi, \quad \pi_{\xi}=-\sqrt{2 m \omega I} \sin \varphi,
$$

we get

$$
H_{\perp}(\varphi, I)=\omega I
$$

which can be dealt with as previously:

The usual quantization procedure for the Hamilton functions (659) or (661) is the standard one, yielding the (Landau) energy levels

$$
E_{\perp, n}=\hbar \omega(n+1 / 2), \quad n=0,1, \ldots
$$


However, quantizing the Hamilton function (666) in the spirit of the present paper yields the Hamilton operator

$$
\hat{H}_{\perp}=\hbar \omega \tilde{K}_{0}
$$

with the possible energy levels

$$
E_{k, n}=\hbar \omega(n+k), n=0,1,2, \ldots
$$

If $k \neq 1 / 2$ the usual Landau energy levels are being shifted to lower or higher values. Whether this really happens has, of course, to be found out experimentally!

\section{Thermodynamics}

Next I collect some thermodynamical properties of a system with energy levels $E_{n}=\hbar \omega(n+k)$ in a heat bath of temperature $k_{B} T \equiv 1 / \beta$ in order to see which quantity depends on the index $k$, and which not! That index $k$ is here, of course, not to be confused with Boltzmann's constant $k_{B}$.

The following simple formulae should be of interest for the interpretation of experiments in preparation for measuring the ground state energy of the HO by means of the AC Josephson effect [40].

From the partition function

$$
Z(\beta ; k)=\sum_{n=0}^{\infty} e^{-\beta \hbar \omega(n+k)}=\frac{e^{-\beta \hbar \omega k}}{1-e^{-\beta \hbar \omega}}
$$

we get the probability to find the system in the $n$th state as

$$
p_{n}(\beta)=e^{-\beta \hbar \omega(n+k)} / Z(\beta ; k)=e^{-\beta \hbar \omega n}\left(1-e^{-\beta \hbar \omega}\right),
$$

which is independent of $k$.

Furthermore we have

Free energy:

$$
\beta F(\beta ; k)=-\ln Z(\beta ; k)=\beta \hbar \omega k+\ln \left(1-e^{-\beta \hbar \omega}\right) .
$$

Internal energy:

$$
U(\beta ; k)=\langle E\rangle(\beta ; k)=-\partial_{\beta} Z(\beta ; k)=\hbar \omega\left(k+\frac{1}{e^{\beta \hbar \omega}-1}\right) .
$$

Energy mean square fluctuations:

$$
(\Delta E)^{2}(\beta)=\partial_{\beta}^{2} \ln Z(\beta ; k)=(\hbar \omega)^{2} \frac{e^{\beta \hbar \omega}}{\left(e^{\beta \hbar \omega}-1\right)^{2}}=k_{B} T^{2} C_{V} .
$$

Entropy:

$$
S(\beta) / k_{B}=\ln Z(\beta ; k)+\beta U=-\ln \left(1-e^{-\beta \hbar \omega}\right)+\frac{\beta \hbar \omega}{e^{\beta \hbar \omega}-1} .
$$

Here $C_{V}$ is the heat capacity of the system at constant volume.

We see that energy fluctuations (heat capacities) and entropy are independent of the index $k$. 


\section{Acknowledgements}

I am indebted to a number of people for stimulating correspondences and discussions: I thank C. Beck, T. Hänsch, W. Ketterle, K.-P. Marzlin and W. Schleich for correspondences concerning possible experimental determinations of the ground state energy of the HO, G. Agarwal, V. Bužek and B. Sanders for correspondences on the experimental production of Perelomov and Barut-Girardello coherent states, and W. Schleich for a correspondence on the experimental generation of Schrödinger-Glauber coherent states. I thank P. Toschek for discussions on the different harmonic traps in quantum optics and A. Ringwald for discussions on the PVLAS experiment.

As before I thank DESY Hamburg, especially the Theory Group, for its generous hospitality after my retirement from the Institute for Theoretical Physics of the RWTH Aachen. The DESY Library has always been very helpful in providing the necessary literature.

Most thanks go to my wife Dorothea who had to endure my many outer and inner absences while I was preoccupied with the present paper!

\section{Appendices}

\section{A Calculating the action variables for certain potentials of 1-dimensional systems}

The calculations of the action variable (81) of subsec. 2.3 .3 for the different potentials discussed there can all be reduced to that of the integral

$$
f(b)=\int_{-b}^{+b} d u \frac{\left(b^{2}-u^{2}\right)^{1 / 2}}{1+u}, \quad 0<b<1,
$$

which may be transformed into [126]

$$
\begin{aligned}
f(b)= & -\int_{-b}^{+b} d u \frac{u}{\left(b^{2}-u^{2}\right)^{1 / 2}}+\int_{-b}^{+b} d u \frac{1}{\left(b^{2}-u^{2}\right)^{1 / 2}} \\
& +\left(b^{2}-1\right) \int_{-b}^{+b} d u \frac{1}{(1+u)\left(b^{2}-u^{2}\right)^{1 / 2}} .
\end{aligned}
$$

Here the first term vanishes (replace $u$ by $-u$ ), the second gives $\pi$ [127], and the last $-(1-$ $\left.b^{2}\right)^{1 / 2} \pi$ [128], so that

$$
f(b)=\left[1-\left(1-b^{2}\right)^{1 / 2}\right] \pi .
$$

In the case of the Morse potential $V_{M o}$ one puts in Eq. (83)

$$
b^{2}=\tilde{E}, \quad u=e^{-\tilde{q}}-1
$$

In the case of the potential $V_{s M o}$ the substitution

$$
u=\tanh \tilde{q},
$$

combined with the observation that

$$
\int_{-b}^{+b} d u \frac{\left(b^{2}-u^{2}\right)^{1 / 2}}{1-u^{2}}=\frac{1}{2} \int_{-b}^{+b} d u\left(b^{2}-u^{2}\right)^{1 / 2}\left(\frac{1}{1+u}+\frac{1}{1-u}\right)=\int_{-b}^{+b} d u \frac{\left(b^{2}-u^{2}\right)^{1 / 2}}{1+u}
$$

works.

For the potential $V_{P T}$ one substitutes $u=\sin \tilde{q}$ and for $V_{c}$ one puts $u=\tilde{q}^{2}+$ const. 


\section{B The covering groups of $\mathrm{SO}^{\uparrow}(1.2)$ and the positive dis- crete series of their irreducible unitary representa- tions}

I have stressed repeatedly in the Sections above that the irreducible unitary representations of those covering groups of $S O^{\uparrow}(1,2)$ or $S p(2, \mathbb{R})$ with a very small Bargmann index $k<1 / 2$ may be of special interest. In this Appendix, therefore, I collect some here relevant properties of those groups and the associated unitary representations. A rather complete list of the literature on the irreducible unitary representations of the group $S O^{\uparrow}(1,2)$ and its covering groups is contained in the Refs. to Appendix B of Ref. [13]. That Appendix contains also a summary of essential properties of those groups.

\section{B.1 The universal covering group of $S O^{\uparrow}(1,2)$}

According to Bargmann [129] the universal covering group $\tilde{G} \equiv \widehat{S O^{\uparrow}(1,2)}$ can be parametrized conveniently by starting from a modified parametrization of the group $S U(1,1)$ as given by the matrices (180), namely by defining

$$
\begin{aligned}
\gamma & =\beta / \alpha, \quad|\gamma|<1) \\
\omega & =\arg (\alpha)
\end{aligned}
$$

with the inverse relations

$$
\begin{aligned}
\alpha & =e^{i \omega}\left(1-|\gamma|^{2}\right)^{-1 / 2},|\gamma|<1 \\
\beta & =e^{i \omega} \gamma\left(1-|\gamma|^{2}\right)^{-1 / 2} .
\end{aligned}
$$

The inequality $|\gamma|<1$ in Eq. (682) follows from the relation $|\alpha|^{2}-|\beta|^{2}=1$.

With

$$
S O^{\uparrow}(1,2)_{[m]}, \quad m \text {-fold covering of } S O^{\uparrow}(1,2),
$$

we have the following relations

$$
\begin{aligned}
S O^{\uparrow}(1,2): & \{(\gamma, \omega),|\gamma|<1, \omega \in(-\pi / 2, \pi / 2]\} \\
S p(2, \mathbb{R}) \cong S U(1,1)=S O^{\uparrow}(1,2)_{[2]}: & \{(\gamma, \omega),|\gamma|<1, \omega \in(-\pi, \pi]\} \\
S O^{\uparrow}(1,2)_{[m]}: & \{(\gamma, \omega),|\gamma|<1, \omega \in(-m \pi / 2, m \pi / 2]\},(68) \\
\tilde{G} \equiv S O^{\uparrow}(1,2)_{[\infty]}: & \tilde{g} \equiv\{(\gamma, \omega),|\gamma|<1, \omega \in \mathbb{R}\} .
\end{aligned}
$$

From the multiplication of the matrices (180) one deduces the group composition law

$$
\left(\gamma_{3}, \omega_{3}\right)=\left(\gamma_{2}, \omega_{2}\right) \circ\left(\gamma_{1}, \omega_{1}\right)
$$

where

$$
\begin{aligned}
\gamma_{3} & =\left(\gamma_{1}+\gamma_{2} e^{-2 i \omega_{1}}\right)\left(1+\gamma_{1}^{*} \gamma_{2} e^{-2 i \omega_{1}}\right)^{-1} \\
\omega_{3} & =\omega_{1}+\omega_{2}+\frac{1}{2 i} \ln \left[\left(1+\gamma_{1}^{*} \gamma_{2} e^{-2 i \omega_{1}}\right)\left(1+\gamma_{1} \gamma_{2}^{*} e^{2 i \omega_{1}}\right)^{-1}\right]
\end{aligned}
$$


For the four subgroups (190) - (193) the new parametrization means

$$
\begin{aligned}
R_{0}: \quad r_{0}= & (\gamma=0, \omega=\theta / 2) \\
& \left(0, \omega_{3}\right)=\left(0, \omega_{2}+\omega_{1}\right) ; \\
A_{0}: \quad a_{0}= & (\gamma=i \tanh (\tau / 2), \omega=0), \tau \in \mathbb{R}, \\
& \left(\gamma_{3}, 0\right)=\left(i \tanh \left[\left(\tau_{2}+\tau_{1}\right) / 2\right], 0\right) ; \\
B_{0}: \quad b_{0}= & (\gamma=\tanh (s / 2), \omega=0), s \in \mathbb{R}, \\
& \left(\gamma_{3}, 0\right)=\left(\tanh \left[\left(s_{2}+s_{1}\right) / 2\right], 0\right) ; \\
N_{0}: \quad n_{0}= & \left(\gamma=\xi\left(\xi^{2}+4\right)^{-1 / 2} e^{-i \omega}, \omega=\arctan (\xi / 2)\right), \xi \in \mathbb{R} .
\end{aligned}
$$

For the universal covering group $\tilde{G}$ the transformations (187) and (186) now take the form

$$
\begin{aligned}
\tilde{I}^{\prime} & =\rho(\tilde{g}, \varphi) \tilde{I}, \rho(\tilde{g}, \varphi)=\left|1+e^{i \varphi} \gamma\right|^{2}\left(1-|\gamma|^{2}\right)^{-1} \\
e^{i \varphi^{\prime}} & =e^{-2 i \omega} \frac{e^{i \varphi}+\gamma^{*}}{1+e^{i \varphi} \gamma}
\end{aligned}
$$

As $\partial \varphi^{\prime} / \partial \varphi=1 / \rho(\tilde{g}, \varphi)$, the equality (189) holds again.

The transformations (699) act, however, not effectively on $\mathcal{S}_{\varphi, \tilde{I}}$ because the (infinite) discrete center

$$
C_{[\infty]}=(0, \omega \in \pi \mathbb{Z}) \subset \tilde{G}
$$

leaves all points $\sigma=(\varphi, \tilde{I})$ invariant. Correspondingly the center

$$
C_{[m]}=(0, \omega=0, \pm \pi, \ldots, \pm m \pi) \subset S O_{[m]}^{\uparrow}(1,2)
$$

of an $m$-fold covering group leaves the points $\sigma$ invariant, too.

With the elements of the group $S U(1,1)$ given by the restriction $-\pi<\omega \leq+\pi, \alpha=\exp (i \omega)\left(1-|\gamma|^{2}\right)^{-1 / 2}, \beta=\gamma \alpha$, the homomorphisms

$$
\begin{aligned}
\Phi_{[\infty] / 2}: \quad \tilde{G} \equiv S O_{[\infty]}^{\uparrow}(1,2) & \rightarrow S U(1,1) \cong S p(2, \mathbb{R}), \\
\Phi_{[2]}: \quad S U(1,1) \cong S p(2, \mathbb{R}) & \rightarrow S O^{\uparrow}(1,2),
\end{aligned}
$$

have the kernels

$$
\operatorname{ker}\left(\Phi_{[\infty] / 2}\right)=2 \pi \mathbb{Z}, \operatorname{ker}\left(\Phi_{[2]}\right)=Z_{2},
$$

respectively, and the composite homomorphism $\Phi_{[\infty]}=\Phi_{[2]} \cdot \Phi_{[\infty] / 2}$ has the kernel

$$
\operatorname{ker}\left(\Phi_{[\infty]}=\Phi_{[2]} \cdot \Phi_{[\infty] / 2}\right)=\pi \mathbb{Z}
$$

As the space $\mathcal{S}_{\varphi, \tilde{I}}^{2}$ is homeomorphic to $\mathbb{R}^{2}-\{0\}=\mathbb{C}-\{0\}$, its universal covering space is given by $\varphi \in \mathbb{R}, \tilde{I} \in \mathbb{R}^{+}$, which is the infinitely sheeted Riemann surface of the logarithm.

The transformations (698) and (699) may be reinterpreted as acting transitively and effectively on that universal covering space. 


\section{B.2 Irreducible unitary representations of the positive discrete se- ries for $k>0$}

I have already mentioned in subsec. 6.4.3 that in the Hilbert space of holomorphic functions on the unit disc $\mathbb{D}=\{z \in \mathbb{C},|z|<1\}$ with the scalar product

$$
(f, g)_{k}=\frac{2 k-1}{\pi} \int_{\mathbb{D}} f^{*}(z) g(z)\left(1-|z|^{2}\right)^{2 k-2} d x d y .
$$

one can define irreducible unitary representations for any $k>0$.

The unitary operators representing the universal covering group in that space are given by

$$
\begin{aligned}
{[U(\tilde{g}, k) f](z) } & =e^{2 i k \omega}\left(1-|\gamma|^{2}\right)^{k}\left(1+\gamma^{*} z\right)^{-2 k} f\left(\frac{\alpha z+\beta}{\beta^{*} z+\alpha^{*}}\right), \\
\tilde{g} & =(\gamma, \omega), \quad\left(\begin{array}{cc}
\alpha & \beta \\
\beta^{*} & \alpha^{*}
\end{array}\right)=\Phi_{[\infty] / 2}(\tilde{g}) \in S U(1,1) .
\end{aligned}
$$

Because $|\gamma z|<1$, the function $\left(1+\gamma^{*} z\right)^{-2 k}$ is, for $k>0$, defined in terms of the series expansion

$$
\left(1+\gamma^{*} z\right)^{-2 k}=\sum_{n=0}^{\infty} \frac{(2 k)_{n}}{n !}\left(-\gamma^{*} z\right)^{n}
$$

The phase factor

$$
e^{2 i k \omega}
$$

in Eq. (707) determines the possible values of $k$ for a given covering group:

For $S O^{\uparrow}(1,2)$ itself we have (see Eq. (687) $) \omega \in \mathbb{R} \bmod \pi$. Requiring the phase factor (1710) to be unique implies $k=1,2, \cdots$.

For $S U(1,1)$ we have $\omega \in \mathbb{R} \bmod 2 \pi$. Uniqueness of the phase factor then requires $k=$ $1 / 2,1,3 / 2, \cdots$.

Uniqness of the same phase factor as to the covering group $S O^{\uparrow}(1,2)_{[m]}$ for which $\omega \in$ $\mathbb{R} \bmod m \pi$ requires

$$
k=\frac{n}{m}, n=1,2, \ldots
$$

For any irrational $k>0$ we get an irreducible representation of the universal covering group $S O^{\uparrow}(1,2)_{[\infty]}$.

\section{Estimates for the ratios $I_{2 k}(2|z|) / I_{2 k-1}(2|z|)$ of modi- fied Bessel functions of the first kind for $k>0$}

In Appendix D.1 of Ref. [13] I deduced the inequality

$$
\rho_{k}(|z|)=I_{2 k}(2|z|) / I_{2 k-1}(2|z|)<1
$$

for the ratio (320) which occurs frequently in expectation values with respect to BarutGirardello coherent states. The argments were: 
It follows from the relation [130]

$$
x \frac{d I_{\nu}}{d x}(x)=\nu I_{\nu}(x)+x I_{\nu+1}
$$

that

$$
I_{\nu+1}(x) / I_{\nu}(x)=\frac{d}{d x} \ln \left(I_{\nu}(x) / x^{\nu}\right)
$$

As $[131]$

$$
I_{\nu}(x)=\frac{x^{\nu}}{2^{\nu} \sqrt{\pi} \Gamma(\nu+1 / 2)} \int_{0}^{\pi} d \theta e^{x \cos \theta} \sin ^{2 \nu} \theta,
$$

we get for the ratio (714)

$$
I_{\nu+1}(x) / I_{\nu}(x)=\frac{\int_{0}^{\pi} d \theta(\cos \theta) e^{x \cos \theta} \sin ^{2 \nu} \theta}{\int_{0}^{\pi} d \theta e^{x \cos \theta} \sin ^{2 \nu} \theta}<1 .
$$

The argument is, however, only valid for $\nu>-1 / 2$, i.e. for $k>1 / 4$, because otherwise the integrals (715) become singular. Thus, the interval $k \in(0,1 / 4]$ has to be treated differently:

For $k=1 / 4$ we have [132]

$$
\rho_{k=1 / 4}(|z|)=\frac{I_{1 / 2}(2|z|)}{I_{-1 / 2}(2|z|)}=\frac{\sinh 2|z|)}{\cosh 2|z|)}=\tanh 2|z|<1 .
$$

For $k \in(0,1 / 4)$, however, the ratio $\rho_{k}$ may become larger than 1 ! This can already be seen from the asymptotic expression (349): If we put $k=1 / 4-\delta, \delta \in(0,1 / 4)$ it takes the form

$$
\rho_{(0<k<1 / 4)}(|z|) \asymp 1+\frac{\delta}{|z|}+\frac{\delta(1+2 \delta)}{4|z|^{2}}+O\left(|z|^{-3}\right), \delta \in(0,1 / 4), \quad|z| \rightarrow \infty .
$$

Now the second and third non-leading terms in the expansion are positive, making the righthand side larger than 1 . The same feature may also been seen in the following way: Because of the relation [133]

$$
I_{2 k-1}(2|z|)=\frac{2 k}{|z|} I_{2 k}(2|z|)+I_{2 k+1}(2|z|)
$$

we have

$$
\rho_{k}(|z|)=\frac{|z|}{2 k+|z| \rho_{k+1 / 2}(|z|)}, \quad \rho_{k+1 / 2}(|z|)=\frac{I_{2 k+1}(2|z|)}{I_{2 k}(2|z|)},
$$

which has the limit

$$
\lim _{k \rightarrow 0} \rho_{k}(|z|)=\frac{I_{0}(2|z|)}{I_{1}(2|z|)}>1 .
$$

Here the right-hand side even diverges for $|z| \rightarrow 0$ ! That the expression (720) can become large for fixed small $|z|$ and decreasing $k$ may also be seen from the approximation (348) which yields

$$
\rho_{k+1 / 2}(|z|) \rightarrow \frac{|z|}{2 k+1}\left(1-\frac{|z|^{2}}{(2 k+1)(2 k+2)}\right) \text { for }|z| \rightarrow 0 .
$$

If $|z|$ is so small that we can neglect the term of order $|z|^{2}$ in the bracket compared to 1 , we get for the relation (720)

$$
\rho_{k}(|z|) \approx \frac{|z|}{2 k+|z|^{2} /(2 k+1)} .
$$


For $k \ll 1 / 2$ and $|z|>2 k+|z|^{2}$ the right-hand side of the expression (1723) becomes larger than 1 .

The possibility that $\rho_{k}(|z|)>1$ for $k \in(0,1 / 4)$ can also be seen from the graphs in Figure 50-1 of Ref. [134].

\section{References}

[1] V.I. Arnold, Mathematical Methods of Classical Mechanics, 2nd edition (Graduate Texts in Mathematics 60; Springer-Verlag, New York etc., 1989), chap. 10, Appendices 7 and 8.

[2] V.I. Arnold, V.V. Kozlov and A.I. Neishtadt, Mathematical Aspects of Classical and Celestial Mechanics, 3rd edition (Encyclopaedia of Mathematical Sciences 3; Dynamical Systems III; Springer-Verlag, Berlin etc., 2006), chaps. 5 and 6.

[3] W. Thirring, Classical Mathematical Physics, Dynamical Systems and Field Theories, 3rd edition (Springer, New York etc., 1997), chaps. 2 and 3.

[4] M. Born, Vorlesungen über Atommechanik, herausg. unter Mitwirkung von F. Hund, Bd. 1 (Struktur der Materie in Einzeldarstellungen II; Verlag Julius Springer, Berlin, 1925)

English translation: The Mechanics of the Atom (G. Bell and Sons, Ltd., London, 1927; reprinted by Frederick Ungar Publ. Co., New York, 1960).

[5] W. Heisenberg, Zeitschr. Physik 33, 879 (1925).

[6] R.S. Mulliken, Phys. Rev. 25, 119 and 259 (1925); the measurements were later improved by F.A. Jenkins and A. McKellar, Phys. Rev. 42, 464 (1932); and the theoretical analysis by J.H. Van Vleck, Journ. Chem. Physics 4, 327 (1936).

[7] G. Herzberg, Molecular Spectra and Molecular Structure I. Spectra of Diatomic Molecules, 2nd edition (Litton Educational Publ., Inc., New York, 1950; afterwards publ. by Van Nostrand Reinhold Co., New York; republished by Krieger Publ. Co., Malabar, Fl., USA, 1989), chaps. III,2, IV,2 and IV,3.

I owe the reference to these discussions by Herzberg on the experimental verifications of the HO ground state energy to one of my teachers, the late Fritz Bopp, who drew our attention to that book during my time as a PhD student at the University of Munich around 1960 .

[8] P.W. Milonni, The Quantum Vacuum, An Introduction to Quantum Electrodynamics (Academic Press, Inc., Boston etc., 1994).

[9] M. Moshinsky and Y.F. Smirnov, The Harmonic Oscillator in Modern Physics (Contemporary Concepts in Physics 9; harwood academic publishers, Australia etc., 1996).

[10] P.A.M. Dirac, Proc. Royal Soc. London, Ser. A, 109, 642 (1925); 110, 561 (1926); 114, 243 (1927).

[11] F. London, Zeitschr. Physik 37, 915 (1926); 40, 193 (1927). 
[12] P. Jordan, Zeitschr. Physik 44, 1 (1927); Jordan here quotes Dirac as telling him that he (Dirac) actually needed only the operator relation $\widehat{\exp i \varphi} \hat{I}-\hat{I} \widehat{\exp i \varphi}=-\hbar \widehat{\exp i \varphi}$ instead of the contradictory $\hat{\varphi} \hat{I}-\hat{I} \hat{\varphi}=i \hbar$ ! Jordan does not mention the additional important condition $\hat{I}>0$.

[13] H.A. Kastrup, Fortschr. Physik 51, 975 (2003); Addendum: ibidem 52, 388 (2004). Quotations in the present paper will refer to the expanded e-print version

arXiv: quant-ph/0307069.

[14] F. London, Zeitschr. Physik 63, 245 (1930); Zeitschr. Physikal. Chemie, Abt. B 11, 222 (1931); Transact. Faraday Soc. 33, 8 (1937); in the present context the second paper is the most essential one.

N.B. It appears to me that F. London's important contributions to the early development of quantum mechanics have been underrated and still are. He was also the first to formulate the gauge principle in quantum mechanics (Zeitschr. Physik 42, 375 (1927)), long before Weyl sanctioned London's quantum mechanical reinterpretation of Weyl's unsuccesful gauge theoretical extension of Einstein's General Relativity (H. Weyl, Die Naturwiss. 19, 49 (1931)). As to the life and scientific work of F. London see K. Gavroglu, Fritz London, a scientific biography (Cambridge Univ. Press, Cambridge etc., 1995).

[15] H.B.G. Casimir, Koninkl. Nederl. Akad. Wetensch. (Amsterdam), Proc. Sec. Sciences 51, 793 (1948).

[16] K.A. Milton, The Casimir Effect, Physical Manifestations of Zero-Point Energy (World Scientific, Singapore etc., 2001).

[17] S. Weinberg, Rev. Mod. Phys. 61, 1 (1989).

[18] S.M. Carroll, Living Reviews: http://www.livingreviews.org/lrr-2001-1 astro-ph/0004075.

[19] P.J.E. Peebles and B. Ratra, Rev. Mod. Phys. 75, 559 (2003).

[20] G.E. Volovik, Ann. Physik (Leipzig) 14, 165 (2005); id., e-print arXiv: gr-qc/0604062.

[21] E.J. Copeland, M. Sami and S. Tsujikawa, Intern. Journ. Mod. Phys. D 15, 1753 (2006) [hep-th/0603057.

[22] T. Padmanabhan, Phys. Reports 380, 235 (2003); id., e-print arXiv: astro-ph/0603114.

[23] N. Straumann, Mod. Phys. Lett. A 21, 1083 (2006); Ann. Physik (Leipzig) 15, 701 (2006).

[24] S. Nobbenhuis, e-print arXiv: gr-qc/0609011 (Ph.D. Thesis, Utrecht University); this paper contains a long list of references.

[25] H.A. Kastrup, Phys. Rev. A 73, 052104 (2006).

[26] M. Bojowald, H.A. Kastrup, F. Schramm and T. Strobl, Phys. Rev. D 62, 044026 (2000). 
[27] L.D. Mlodinow and N. Papanicoulaou, Ann. Phys. (N.Y.) 128, 314 (1980); see also

C.C. Gerry, J. Phys. A: Math. Gen. 16, L1 (1983);

J. Katriel, A.I. Solomon, G. D’Ariano and M. Rasetti, Phys. Rev. D 34, 2332 (1986);

H. Bacry, Journ. Mathem. Phys. 31, 2061 (1990);

C.C. Gerry and R. Grobe, Quantum Semiclass. Opt. 9, 59 (1997);

A. Wünsche, Acta physica slov. 49, 771 (1999); Journ. Opt. B: Quantum Semiclass. Opt. 5, S429 (2003).

[28] C.J. Isham in: Relativity, Groups and Topology II (Les Houches Session XL, 1983), edited by B.S. Dewitt and R. Stora (North-Holland, Amsterdam etc., 1984), p. 1059.

[29] V. Guillemin and S. Sternberg, Symplectic techniques in physics (Cambridge University Press, Cambridge etc., 1984; paperback edition: 1990).

[30] Refs. [13,26]

[31] E. Zavattini et al., Phys. Rev. Lett. 96, 110406 (2006); as to previous theoretical and experimental work which led to this experiment see the literature quoted in the paper. For very recent critical evaluations of that experiment see

S.L. Adler, Journ. Phys. A: Math. Theor. 40, F143 (2007);

A.C. Melissinos, e-print arXiv: hep-ph/0702135.

[32] J. Doyle, B. Friedrich, R.V. Kraus and F. Masnou-Seeuws, Europ. Phys. Journ. D 31, 149 (2004).

[33] H. Feshbach, Ann. Phys. (N.Y.) 5, 357 (1958); 19, 287 (1962); id., Theoretical Nuclear Physics (John Wiley \& Sons, N.Y., 1992) chap. III;

in the literature on potential scattering theory the "Feshbach" resonances are called "virtual" or "anti-" bound states. They correspond to zeros of the Jost-function on the negative imaginary axis of the complex momentum plane (see, e.g.

S.T. Ma, Rev. Mod. Phys. 25, 853 (1953);

V. de Alfaro and T. Regge, Potential Scattering (North-Holland Publ. Co., Amsterdam, 1965) chap. 7.5.;

J.R. Taylor, Scattering Theory: The Quantum Theory on Nonrelativistic Collisions (John Wiley \& Sons, Inc., New York etc., 1972) chap. 13);

theoretical papers on Feshbach resonances at very low temperatures include:

E. Tiesinga, A.J. Moerdijk, B.J. Verhaar and H.T.C. Stoof, Phys. Rev. A 46, R1167 (1992); E. Tiesinga, B.J. Verhaar and H.T.C. Stoof, Phys. Rev. A 47, 4114 (1993);

B. Marcelis, E.G.M. van Kempen, B.J. Verhaar and S.J.J.M.F. Kokkelmans, Phys. Rev. A 70, 012701 (2004);

J.N. Milstein, PhD Thesis University of Colorado, Boulder, 2004; available under https://jilawww.colorado.edu/pubs/thesis/milstein;

V.A. Yurovsky, Phys. Rev. A 71, 012709 (2005);

S. Dürr et al., Phys. Rev. 72, 052707 (2005);

N. Nygaard, B.I. Schneider and P.S. Julienne, Phys. Rev. A 73, 042705 (2006);

the later papers mentioned here contain further Refs. to earlier ones.

[34] Early experimental observations of Feshbach resonances in BEC are

S. Inouye et al., Nature 392, 151 (1998); 
Ph. Courteille et al., Phys. Rev. Lett. 81, 69 (1998);

J.L. Roberts et al., Phys. Rev. Lett. 81, 5109 (1998);

more recent experimental papers with further references are

M.W. Zwierlein et al., Phys. Rev. Lett. 92, 120403 (2004);

C.H. Schunck et al., Phys. Rev. A 71, 045601 (2005);

T. Volz et al., Nature Physics 2, 692 (2006).

[35] Properties of lower dimensional gases at low temperatures are discussed in the contributions to the school Quantum Gases in Low Dimensions, (QGLD 2003) from April 2003 at the Centre de physique des Houches; Proc. edited by L. Pricoupenko, H. Perrin and M. Olshanii, Journ. Physique IV (Proceedings) 116 (2004).

[36] Reviews are D. Leibfried, R. Blatt, C. Monroe and D. Wineland, Rev. Mod. Phys. 75, 281 (2003);

J. Eschner, G. Morigi, F. Schmidt-Kaler and R. Blatt, Journ. Opt. Soc. Am. B 20, 1003 (2003);

for a discussion of theoretical aspects as to Paul traps see

W.P. Schleich, Quantum Optics in Phase Space (WILEY - VCH Verlag, Berlin etc., 2001), chap. 17.

[37] Reviews:

Laser Manipulations of Atoms and Ions, Proc. Intern. School of Physics "Enrico Fermi", Course CXVIII (1991), edited by E. Arimondo, W.D. Phillips and F. Strumia (NorthHolland, Elsevier Science Publ., Amsterdam, 1992);

J.M. Raimond, M. Brune and S. Haroche, Rev. Mod. Phys. 73, 565 (2001);

P. Domokos and H. Ritsch, Journ. Opt. Soc. Am. 20, 1098 (2003);

Several contributions in Ref. [35]

Special issue: Atoms, Quanta and Relativity - a century after Einstein's miraculous year, edited by T.W. Hänsch, H. Schmidt-Böcking and H. Walther, Journ. Physics B: Atomic, Molecular and Optical Physics 38, No. 9 (2005)

P. Treutlein et al., Fortschr. Physik 54, 702 (2006) quant-ph/0605163];

A. Ashkin, Optical Trapping and Manipulations of Neutral Particles Using Lasers, A Reprint Volume with Commentaries (World Scientific Publ. Co. Pte. Ltd., New Jersey etc., 2006).

[38] A.S. Parkins and D.F. Walls, Phys. Reports 303, 1 (1998);

Bose-Einstein Condensation in Atomic Gases, Proc. Intern. School of Physics "Enrico Fermi", Course CXL (1998), edited by M. Inguscio, S. Stringari and C.E. Wieman (IOS Press, Amsterdam, 1999);

A.J. Leggett, Rev. Mod. Phys. 73, 307 (2001);

E.A. Cornell and C.E. Wieman, Rev. Mod. Phys. 74, 875 (2002);

W. Ketterle, Rev. Mod. Phys. 74, 1131 (2002);

Several review articles in Nature 416, 206-248 (2002);

K. Bongs and K. Sengstock, Rep. Progr. Phys. 67, 907 (2004);

O. Morsch and M. Oberthaler, Rev. Mod. Phys. 78, 179 (2006).

[39] W. Ketterle and N.J. van Druten, Phys. Rev. A 54, 656 (1996);

D.S. Petrov, G.V. Shlyapnikov and J.T.M. Walraven, Phys. Rev. Lett. 85, 3745 (2000);

K. Bongs et al., Phys. Rev. A 63, 031600(R) (2001); 
Görlitz et al., Phys. Rev. Lett. 87, 130402 (2001);

M. Greiner et al., Phys. Rev. Lett. 87, 160405 (2001);

S. Dettmer et al., Phys. Rev. Lett. 87, 160406 (2001).

[40] C. Beck, Journ. Physics: Conf. Series 31, 123 (2006); C. Beck and M.C. Mackey, Physica A 379, 101 (2007);

these papers contain references to previous and future experimental work.

[41] See Appendix C of Ref. [13] and the literature on the symplectic groups $S p(2 n, \mathbb{R})$ and the discrete series of their irreducible unitary representations quoted there. See also R.C. King and B.G. Wybourne, Journ. Phys. A: Math. Gener. 18, 3113 (1985).

[42] V. Bargmann, in: Analytical methods in mathematical physics, edited by R.P. Gilbert and R.G. Newton (Based on the conference held at Indiana University, Bloomington, Indiana, June 2-6, 1968; Gordon and Breach Science Publ., N.Y., London and Paris, 1970), p. 27;

a famous theorem by Harish-Chandra says that a non-compact semisimple group has discrete series of irreducible unitary representations iff the rank of the maximal compact subgroup is equal to the rank of the group itself (Acta Mathem. 116, 1 (1966); theorem $13)$. This is the case for the symplectic groups $S p(2 n, \mathbb{R})$.

Discrete series and Harish-Chandra's work on them play a major role in the textbook A.W. Knapp, Representation Theory of Semisimple Groups, an Overview based on Examples (Princeton University Press, Princeton, N.J., 1986).

[43] D. Husemoller, Fibre Bundles, 2nd edition (Graduate Texts in Mathematics 20; Springer-Verlag, New York etc., 1975), pp. 92-93.

[44] J.-M. Souriau, Structure of Dynamical Systems, A Symplectic View of Physics (Progress in Mathematics 149; Birkhäuser, Boston etc., 1997; English translation of the French edition Structure des systémes dynamiques, Dunod, Paris, 1969).

[45] J.E. Marsden and T.S. Ratiu, Introduction to Mechanics and Symmetry, A Basic Exposition of Classical Mechanical Systems, 2nd edition (Texts in Applied Mathematics 17; Springer-Verlag, New York etc., Corrected printing, 2003).

[46] I.S. Gradshteyn and I.M. Ryzhik, Table of Integrals, Series, and Products, 4th edition (Academic Press, New York and London, 1965), p. 148 , formula 2.5533.

[47] Ref. [46], p. 147 , formula 2.5513.

[48] Many such examples are also discussed in O.L. De Lange and R.E. Raab, Operator Methods in Quantum Mechanics (Clarendon Press, Oxford, 1991).

[49] P.M. Morse, Phys. Rev. 34, 57 (1929); for the radial ( $s$-wave) case $q=r \geq 0$ one has to choose appropriate boundary conditions at $r=0$ ( D. ter Haar, Phys. Rev. 70, 222 (1946)). 
[50] N. Rosen and P.M. Morse, Phys. Rev. 42, 210 (1932);

L.D. Landau and E.M. Lifshitz, Quantum Mechanics, Non-Relativisic Theory, 3rd edition (Vol. 3 of Course of Theoretical Physics, Butterworth-Heinemann (Elsevier Science), Amsterdam etc., 1977, 1991), p. 73 (problem 5);

M.M. Nieto and L.M. Simmons, Jr., Phys. Rev. D 20, 1342 (1979).

[51] G. Pöschl und E. Teller, Zeitschr. Physik 83, 143 (1933).

[52] P. Camiz, A. Gerardi, C. Marchioro, E. Presutti and E. Scacciatelli, Journ. Math. Phys. 12, 2040 (1971);

E. Onofri and M. Pauri, Lett. Nuovo Cim. 3, 35 (1972);

P. Cordero, S. Hojman, P. Furlan and G.C. Ghirardi, Nuovo Cim. 3 A, 807 (1971);

V.V. Dodonov, I.A. Malkin and V.I. Man'ko, Physica 72, 597 (1974);

V. De Alfaro and S. Fubini and G. Furlan, Nuovo Cim. 34 A, 569 (1976);

M.M. Nieto and L.M. Simmons, Jr., Phys. Rev. D 20, 1332 (1979); M. Nieto, Phys. Rev. D 22, 391 (1980).

[53] P.M. Morse, Ref. [49];

Landau/Lifshitz, Ref. [50], p. 72 (problem 4);

P. Cordero and S. Hojman, Lett. Nuovo Cim. 4, 1123 (1970);

M.M. Nieto and L.M. Simmons, Jr., Phys. Rev. D 19, 438 (1979); 20, 1342 (1979);

C.C. Gerry, Phys. Rev. A 33, 2207 (1986);

J.P. Dahl and M. Springborg, Journ. Chem. Phys. 88, 4535 (1988);

S. Kais and R.D. Levine, Phys. Rev. A 41, 2301 (1990).

[54] M.M. Nieto and L.M. Simmons, Jr., Phys. Rev. 20, 1332 (1979);

V.P. Gutschick and M.M. Nieto, Phys. Rev. D 22, 403 (1980);

A. Wünsche, Journ. Opt. B: Quantum Semiclass. Opt. 4, 359 (2002).

[55] See Landau/Lifshitz, Ref. [50], pp. 72-74.

[56] A.A. Kirillov, Elements of the Theory of Representations (Grundlehren der mathematischen Wissenschaften 220; Springer-Verlag, Berlin, Heidelberg and N.Y., 1976; translation of the Russian edition from 1972), pp. 287-290;

R. Howe, Bull. Amer. Math. Soc. (New Ser.) 3, 821 (1980);

id., in: The Mathematical Heritage of Hermann Weyl, edited by R.O. Wells, Jr. (Proc. Symposia Pure Mathem. 48; Amer. Math. Soc., Providence, R.I., 1988), p. 61;

G.B. Folland, Harmonic Analysis in Phase Space (Ann. Mathem. Studies 122; Princeton University Press, Princeton, N.J., 1989);

E.M. Stein, Harmonic Analysis: Real-Variable Methods, Orthogonality, and Oscillatory Integrals (Princeton University Press, Princeton, N.J., 1993, 2nd printing 1995), chaps. XII and XIII.

[57] See, e.g. Ref. [45], p. 68.

[58] See subsec. 6.3 of Ref. [13] and the literature quoted there.

[59] A. Weil, Acta mathem. 111, 143 (1964). 
[60] A selection of books and articles containing discussions of metaplectic groups and references to original papers is:

D. Shale, Trans. Amer. Math. Soc. 103, 149 (1962);

G. Lion and M. Vergne, The Weil representation, Maslov index and Theta series (Progress in Mathematics 6; Birkhäuser, Boston, 1980);

R. Howe, Ref. [56];

V. Guillemin and S. Sternberg, Ref. [29];

G.B. Folland, Ref. [56];

R. Simon and N. Mukunda, in: Symmetries in Science VI, From the Rotation Group to Quantum Algebras, edited by B. Gruber (Proceed. Symposium Bregenz (Austria) August 1992; Plenum Press, New York and London, 1993), p. 659;

Arvind, B. Dutta, C.L. Mehta and N. Mukunda, Phys. Rev. A 50, 39 (1994);

E.M. Stein, Ref. [56].

[61] See subsec. 6.1 of Ref. [13].

[62] See subsecs. 6.2 and 6.3 of Ref. [13].

[63] M. Bojowald and T. Strobl, Journ. Math. Phys. 41, 2537 (2000); Intern. Journ. Mod. Phys. D 12, 713 (2003).

[64] For more details see Ref. [13], Appendix B.

[65] See Ref. [26], sec. III.

[66] As to orbifolds see subsecs. 1.4 and A.3 of Ref. [13] and the references quoted there.

[67] See subsec. 1.3 of Ref. [13].

[68] See the literature quoted in Appendix B of Ref. [13].

[69] As to descriptions of irreducible unitary representations of the same group in terms of a "continuous" basis see

N. Mukunda, Journ. Math. Phys. 8, 2210 (1967);

J.G. Kuriyan, N. Mukunda and E.C.G. Sudarshan, Journ. Mathem. Phys. 9, 2100 (1968);

N. Mukunda, Journ. Mathem. Phys. 10, 2086 and 2092 (1969);

G. Lindblad and B. Nagel, Ann. Inst. Henri Poincaré 13, 27 (1970);

W. Montgomery and L. O’Raifeartaigh, Journ. Math. Phys. 15, 380 (1974).

[70] For a comprehensive survey as to the literature on squeezed states see

V.V. Dodonov, Journ. Opt. B: Quantum Semiclass. Opt. 4, R1 (2002).

[71] See chap. 3 of Ref. [13] and the literature quoted there.

[72] W. Thirring and B. Touschek, Philos. Mag. (7th Ser.) 42, 244 (1951);

R.J. Glauber, Phys. Rev. 84, 395 (1951);

K.O. Friedrichs, Mathematical Aspects of the Quantum Theory of Fields (Interscience Publ., Inc., New York, 1953), part III;

J.R. Klauder, Ann. Phys. (N.Y.) 11, 123 (1960);

R.J. Glauber, Phys. Rev. 131, 2766 (1963);

P. Carruthers and M.M. Nieto, Amer. Journ. Phys. 33, 537 (1965). 
[73] M. Sargent III, M.O. Scully and W.E. Lamb, Jr., Laser Physics (Addison-Wesley Publ. Co., Reading, Mass. etc., 1974), chap. 15-2;

Fam Le Kien, M.O. Scully and H. Walther, Found. Physics 23, 177 (1993);

for a recent discussion as to experimental creations of coherent states see chap. VI. A. of the review by Leibfried et al. quoted in Ref. [36].

[74] D.F. Walls and G.J. Milburn, Quantum Optics (Springer-Verlag, Berlin etc., 1994), chap. 12.2;

L. Mandel and E. Wolf, Optical Coherence and Quantum Optics (Cambridge University Press, Cambridge etc., 1995), chap. 20.4;

M.O. Scully and M.S. Zubairy, Quantum Optics (Cambridge University Press, Cambridge (UK) etc., 1997), chap. 11.2;

W.E. Lamb, W.P. Schleich, M.O. Scully and C.H. Townes, Rev. Mod. Phys. 71, S263 (1999).

[75] A. Perelomov, Generalized Coherent States and Their Applications (Springer-Verlag, Berlin etc., 1986), chap. 5.

[76] Ref. [13], subsec. 6.1.

[77] P. Shanta, S. Chaturvedi, V. Srinivasan, G.S. Agarwal and C.L. Mehta, Phys. Rev. Lett. 72, 1447 (1994);

X.-G. Wang, Intern. Journ. Mod. Phys. B 14, 1093 (2000);

A. Wünsche, Journ. Opt. B: Quantum Semiclass. Opt. 4, 359 (2002); 5, S429 (2003); these papers contain many references to earlier work. For a non-grouptheoretical approach to generating coherent states by non-linear functions of the conventional creation and annihilation operators see

R.L. de Matos Filho and W. Vogel, Phys. Rev. A 54, 4560 (1996);

V.I. Man'ko, G. Marmo, E.C.G. Sudarshan and F. Zaccaria, in: Proceed. of the IV. Wigner Symposium, Guadalajara 1995, edited by N. Atakishiyev, T. Seligman and K.B. Wolf (World Scientific, Singapore, 1996), p. 421; Physica Scr. 55, 528 (1997);

S. Sivakumar, Journ. Opt. B: Quantum Semiclass. Opt. 2, R61 (2000);

X. Wang, Canad. Journ. Phys. 79, 833 (2001).

[78] Ref. [13], subsec. 3.1.

[79] A. Erdélyi et al. (Eds.), Higher Transcendental Functions II (McGraw-Hill Book Co., Inc., New York etc., 1953), chap. VII.

[80] See chap. 3 of Ref. [13] and the literature quoted there.

[81] A. Erdélyi et al. (Eds.), Higher Transcendental Functions I (McGraw-Hill Book Co., Inc., New York etc., 1953), here p. 9.

[82] V. Bargmann, Commun. Pure and Appl. Math. 14, 187 (1961); 20, 1 (1967).

[83] I. Segal, Mathematical Problems of Relativistic Physics, Lectures in Applied Mathematics II, Proceed. Summer Seminar, Boulder, Colorado, 1960, edited by M. Kac (Amer. Math. Soc., Providence, R.I., 1963); idem, Illinois Journ. Math. 6, 500 (1962); 
For a recent review of Bargmann's and Segal's work see B.C. Hall, Contemp. Mathem. 260, 1 (2000) quant-ph/9912072.

[84] V. Fock, Zeitschr. Physik 49, 339 (1928).

[85] G.M. D’Ariano, M. Vasilyev and P. Kumar, Phys. Rev. A 58, 636 (1998);

M. Vasilyev, S.-K. Choi, P. Kumar and G.M. D'Ariano, Opt. Lett. 23, 1393 (1998);

G.M. D’Ariano, M. Sacchi and P. Kumar, Phys. Rev. A 61, 013806 (1999);

M. Vasilyev, S.-K. Choi, P. Kumar and G.M. D'Ariano, Phys. Rev. Lett. 84,2354 (2000);

H. Cao, Y. Ling, J.Y. Xu and C.Q. Cao and P. Kumar, Phys. Rev. Lett. 86, 4524 (2001);

P. Voss, T.-G. Noh, S. Dugan, M. Vasilyev, P. Kumar and G.M. D’Ariano, Journ. Mod.

Opt. bf 49, 2289 (2002);

P.L. Voss, R. Tang and P. Kumar, Opt. Lett. 28, 549 (2003).

[86] D.F. Walls and G.J. Milburn, Ref. [74], chap. 5;

L. Mandel and E. Wolf, Optical Coherence and Quantum Optics (Cambridge University

Press, Cambridge etc., 1995), chap. 22;

P. Hariharan and B.C. Sanders, Progr. Optics 36, 49 (1996);

M.O. Scully and M.S. Zubairy, Ref. [74], chap. 16;

V. Peřinová, A. Lukš and J. Peřina, Phase in Optics (World Scientific Publ. Co., Singapore, 1998), chap. 5.4;

W. Vogel, D.-G. Welsch and S. Wallentowitz, Quantum Optics. An Introduction, 2nd edition (Wiley-VHC Verlag, Weinheim, 2001), chap. 8;

R.R. Puri, Mathematical Methods of Quantum Optics (Springer Series in Optical Sciences 79, Springer, Berlin etc., 2001), chap. 7;

U. Leonhardt, Rep. Prog. Phys. 66, 1207 (2003);

H.-A. Bachor and T.C. Ralph, A Guide to Experiments in Quantum Optics, 2nd revised and enlarged edition (Wiley-VCH Verlag, Weinheim, 2004), chaps. 6.2 and 9.

[87] G. Dattoli, J.C. Gallardo and A. Torre, Rivista Nuovo Cim. 11, article No. 11 (1988).

[88] K. Wódkiewicz and J.H. Eberly, Journ. Opt. Soc. Amer. B 2, 458 (1985);

K. Wódkiewicz, Journ. Mod. Opt. 34, 941 (1987);

C.C. Gerry, Phys. Rev. A 31, 2721 (1985); 35, 2146 (1987); Journ. Opt. Soc. Amer. B

8,685 (1991); C.C. Gerry and J. Kiefer, Journ. Phys. A: Math. Gener. 24, 3513 (1991);

G.S. Agarwal, Phys. Rev. Lett. 57, 827 (1986); Journ. Opt. Soc. Amer. B 5, 1940 (1988);

P.K. Aravind, Journ. Opt. Soc. Amer. B 5, 1545 (1988);

V. Bužek, Phys. Rev. A 39, 3196 (1989); 39, 5432 (1989); Phys. Lett. A 136, 188 (1989); Acta phys. slov. 39, 344 (1989); Journ. Mod. Opt. 37, 303 (1990);

Z.W. Gortel and Ł.A. Turski, Phys. Rev. A 43, 3221 (1991);

V. Penna, Ann. Phys. (N.Y.) 245, 389 (1996).

[89] R.W. Boyd, Nonlinear Optics, 2nd edition (Academic Press, Elsevier, San Diego, 2003); see also the textbooks of the previous Ref. [86] and

A. Yariv, Optical Electronics in Modern Communications, 5th edition (Oxford University Press, New York and Oxford, 1997). 
[90] M. Hillery, Opt. Commun. 62, 135 (1987); Phys. Rev. A 36, 3796 (1987); Phys. Rev. A 40, 3147 (1989);

C.C. Gerry and E.R. Vrscay, Phys. Rev. 37, 1779 (1988).

[91] R.W. Boyd, Ref. [89], chap. 4;

A. Yariv, Ref. [89], chap. 17.

[92] See the Refs. of chap. 16 (pp. 484-486) in the textbook by Scully and Zubairy, Ref. [74] above.

[93] See chap. 6.4 of Ref. [13] and the literature quoted there.

[94] B. Yurke, S.L. McCall and J.R. Klauder, Phys. Rev. A 33, 4033 (1986);

U. Leonhardt, Phys. Rev. A 49, 1231 (1994);

C. Brif and Y. Ben-Aryeh, Quantum Semiclass. Opt. 8, 1 (1996);

C. Brif and A. Mann, Phys. Lett. A 219, 257 (1996); Phys. Rev. A 54, 4505 (1996);

Y. Ben-Aryeh, D. Ludwin and A. Mann, Journ. Opt. B: Quantum Semiclass. Opt. 3, 138 (2001)

B.C. Sanders and D.A. Rice, Phys. Rev. A 61, 013805 (1999); Opt. Quantum Electron. 31, 525 (1999);

C.C. Gerry, Phys. Rev. A 59, 4095 (1999);

V. Peřinová, A. Lukš and J. Křepelka, Journ. Opt. B: Quantum Semiclass. Opt. 2, 81 (2000).

[95] G.S. Agarwal, papers quoted in Ref. [88];

G.S. Prakash and G.S. Agarwal, Phys. Rev. A 50, 4258 (1994); 52, 2335 (1995);

B.A. Bambah and G.S. Agarwal, Phys. Rev. A 51, 4918 (1995);

A. Joshi and R.R. Puri, Phys. Rev. A 42, 4336 (1990);

C.C. Gerry and R.F. Welch, Journ. Opt. Soc. Amer. B 8, 868 (1991);

C.C. Gerry and R. Grobe, Phys. Rev. A 51, 1698 (1995);

S.-C. Gou, J. Steinbach and P.L. Knight, Phys. Rev. A 54, 4315 (1996);

C.C. Gerry, S.-C. Gou and J. Steinbach, Phys. Rev. A 55, 630 (1997);

M.S. Abdalla, F.A.A. El-Orany and J. Peřina, Acta phys. slovaca 50, 613 (2000);

X. Wang, B.C. Sanders and S.-h. Pan, Journ. Phys. A: Math. Gener. 33, 7451 (2000);

G.S. Agarwal and A. Biswas, Journ. Opt. B: Quantum Semiclass. Opt. 7, 350 (2005);

New Journ. Phys. 7, 211 (2005).

[96] V. Bužek, papers quoted in Ref. [88];

C.C. Gerry and R. Grobe, Ref. [27]; Quantum Semiclass. Opt. 9, 59 (1997);

G. Ariunbold and J. Peřina, Acta phys. slovaca 48, 315 (1998).

[97] See the literature quoted in Appendix C.5.1 of Ref. [13];

S.D. Bartlett, D.A. Rice, B.C. Sanders, J. Daboul and H. de Guise, Phys. Rev. A 63, 042310 (2001).

[98] See also chap. 4 of Ref. [13] (e-print version).

[99] See, e.g. A. Messiah, Quantum Mechanics, vol. I (North-Holland Publ. Co., Amsterdam, 1961), chap. XII and Appendix B III. 
[100] E. Hille, Analytic Function Theory II, 2nd edition (Chelsea Publ. Co., New York, 1987), chap. 14;

B. Ya. Levin, in collaboration with Yu. Lyubarskii, M. Sodin and V. Tkachenko, Lectures on Entire Functions (Transl. Mathem. Monographs 150, Amer. Math. Soc., Providence, R.I., 1996).

[101] N.G. de Bruijn, Asymptotic Methods in Analysis (Bibliotheca Mathematica IV; NorthHolland Publ. Co., Amsterdam, 1958), chaps. 5 and 6;

N. Bleistein and R.A. Handelsman, Asymptotic Expansions of Integralss (Dover Publ., Inc., New York, 1975, reprinted 1986), chap. 7;

J.D. Murray, Asymptotic Analysis (Appl. Mathem. Sciences 48, Springer, New York etc., 1984), chap. 3.

[102] Here one puts $\zeta^{n} /(n !)^{1 / \rho}=\exp [n \ln \zeta-(1 / \rho) \ln \Gamma(n+1)]$, takes for $\ln \Gamma(x+1)$ the usual asymptotic expansion (see, e.g. Ref. [46], subsec. 8.34) and then determines the saddle point.

[103] M.A. Evgrafov, Asymptotic Estimates and Entire Functions (Russian Tracts on Advanced Mathematics and Physics IV; Gordon and Breach, Science Publ., Inc., New York, 1961), chap. III, here pp. 149-151;

The 3rd enlarged and improved Russian edition (Asimptotičeskie ocenki i celye funkcii, Moskva, 1979) contains also the estimate (512), with the factor $\sqrt{\rho}$ missing (chap. IV, pp. 289-294).

For real $\zeta>0$ the asymptotic expansion (512) is contained in

M.É. Le Roy, Bull. Sciences Mathém. (2. Sér.) 24, 245 (1900); see also

G.H. Hardy, Orders of Infinity (Cambridge Tracts Mathem. and Mathemat. Physics No. 12; Cambridge Univ. Press, 1910), here p. 55.

[104] G.N. Watson, A Treatise on the Theory of Bessel Functions, 2nd edition (Cambridge Univ. Press, Cambridge, 1966), p. 181, formula (4).

[105] Ref. [46], formula 3.6132.

[106] See, e.g. J.B. Conway, Functions of One Complex Variable (Graduate Texts in Mathem. 11; Springer-Verlag, New York etc., 1973), chap. X.

[107] G.S. Agarwal, Phys. Rev. A 45, 1787. (1992); Opt. Commun. 100, 479 (1993);

A. Vourdas, Phys. Rev. A 45, 1943 (1992).

[108] Ref. [81], here p. 80.

[109] H.S. Shapiro and A.L. Shields, Mathem. Zeitschr. 80, 217 (1962);

P.J. Sally, Jr., Journ. Funct. Analysis 6, 441 (1970);

C.P. Boyer and K.B. Wolf, Journ. Mathem. Phys. 16, 1493 (1975);

Ref. [26], chap. V, C; Ref. [13], chap. 4.5.

[110] E.T. Whittaker and G.N. Watson, A Course of Modern Analysis, 4th edition (Cambridge Univ. Press, Cambridge, 1969), p. 21.

[111] V. Bargmann, first of the Refs. [82], pp. 203-204. 
[112] Ref. [79], chap. X.12

[113] Ref. [26], chap V, secs. D-F; in Eq. (103) there one has $2 t=u$.

[114] E.C. Titchmarsh, Introduction to the Theory of Fourier Integrals, 2nd edition (Oxford Univ. Press, London etc., 1950), subsec. 3.16;

R. Courant and D. Hilbert, Methods of Mathematical Physics I (Interscience, New York, 1953), pp. 103-105.

[115] Ref. [81], p. 251.

[116] See subsec. 3.6. of the following review: K.A. Milton, Journ. Physics A: Math. Gener. 37, R209 (2004).

[117] J. Schwinger, Lett. Mathem. Phys. 1, 43 (1975);

J. Schwinger, L.L. DeRaad, Jr., and K.A. Milton, Ann. Phys. (N.Y.) 115, 1 (1978); see also

R.L. Jaffe, Phys. Rev. D 72, 021301(R) (2005);

A. Scardicchio and R.L. Jaffe, Nucl. Phys. B 743, 249 (2006);

A. Lambrecht, P.A. Maia Neto and S. Reynaud, New Journ. Physics 8, 243 (2006);

N.A. Kawakami, M.C. Nemes and W.F. Wreszinski, e-print arXiv: math-ph/0611025;

S.K. Lamoreaux, Physics Today, Febr. 2007, p. 40.

[118] See, e.g.

W.K.H. Panofsky and M. Phillips, Classical Electricity and Magnetism, 2nd edition (Addison-Wesley Publ. Co., Reading, Mass., etc., 1972), sec. 24-4;

W.H. Louisell, Quantum Statistical Properties of Radiation (John Wiley \& Sons, New York etc., 1973), sec. 4.3;

L. Mandel and E. Wolf, Optical Coherence and Quantum Optics (Cambridge University Press, Cambridge etc., 1995), sec. 10.2;

W.P. Schleich, Ref. [36], secs. 10.2 and 10.3.

[119] In this context see also

J.D. Jackson, Classical Electrodynamics, 3rd edition (John Wiley \& Sons, Inc., New York etc., 1999), secs. 7.1 and 7.2.

[120] See the especially lucid discussion by Louisell, Ref. [118].

[121] D.N. Spergel et al., e-print arXiv: astro-ph/0603449.

[122] L.D. Baron, Molecular Light Scattering and Optical Activity (Cambridge Univ. Press, Cambridge etc., 1982);

M. Born and E. Wolf, Principles of Optics, 7th (expanded) edition (Cambridge Univ. Press, Cambridge etc., 1999), chap. XV;

Encyclopedia of Applied Physics, edited by G.L. Trigg et al., vol. 9, p. 157; vol. 12, p. 285; vol. 14, p. 341 (VCH Publ., Inc., New York etc., 1994-96).

[123] G. Cantatore (PVLAS Collaboration), Talk given at the Workshop "Axions at the Institute for Advanced Study, Oct. 20-23, 2006"; slides available under http://www.sns.ias.edu/ axions/schedule.shtml. 
[124] Molecular Hydrogen in Space (Contributions Intern. Conf. on $\mathrm{H}_{2}$ in Space, Paris, Sept. 1999), edited by F. Combes and G. Pineau des Forets (Cambridge Contemporary Astrophysics Series, Cambridge Univ. Press, Cambridge, 2001);

S. Lepp, P.C. Stancil and A. Dalgarno, Journ. Phys. B: At. Mol. Opt. Phys. 35, R57 (2002).

[125] L. Landau, Zeitschr. Physik 64, 629 (1930);

M.H. Johnson and B.A. Lippmann, Phys. Rev. 76, 828 (1949);

W. Thirring, Quantum Mathematical Physics, 2nd edition, corr. and rev. 2nd printing (Springer-Verlag, Heidelberg etc., 2003), pp. 109-110; 410-412.

[126] Ref. [46], p. 89, formula 2.2822.

[127] Ref. [46], p. 81, formula 2.261.

[128] Ref. [46], p. 89, formula 2.281.

[129] V. Bargmann, Ann. Math. 48, 568 (1947).

[130] Ref. [104], p. 79, formula (4).

[131] Ref. [104], p. 79, formula (9).

[132] Ref. [104], p. 80, formulae (10) and (11) for $n=0$.

[133] Ref. [104], p. 79, formula (1).

[134] J. Spanier and K.B. Oldham, An Atlas of Functions (Hemisphere Publ. Corpor. - Taylor \& Francis Group - , New York etc., 1987), Fig. 50-1 on p. 490. 